PAPER

Waves in hyperbolic and double negative metamaterials including rogues and solitons

To cite this article: A D Boardman et al 2017 Nanotechnology 28444001

View the article online for updates and enhancements.
Related content

Nonlinear silicon photonics M Borghi, C Castellan, S Signorini et al.

- Surface plasmon enhancement of spontaneous emission in graphene waveguides

Mauro Cuevas

Designing an ultrafast laser virtual laboratory using MATLAB GUIDE F Cambronero-López, A I Gómez-Varela and C Bao-Varela

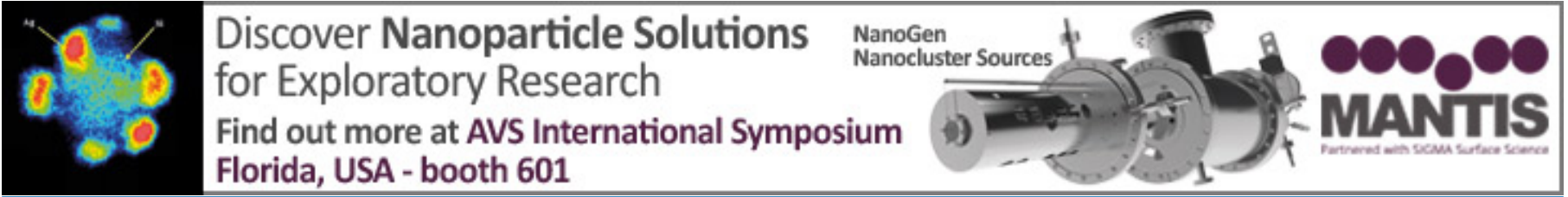




\title{
Waves in hyperbolic and double negative metamaterials including rogues and solitons
}

\author{
A D Boardman ${ }^{1,11}$, A Alberucci ${ }^{2}$, G Assanto $^{2,3}$, V v Grimalsky $^{4}$, B Kibler ${ }^{5}$, \\ J McNiff $^{6}$, I S Nefedov ${ }^{7,8}$, Yu G Rapoport ${ }^{9}$ and C A Valagiannopoulos ${ }^{10}$ \\ ${ }^{1}$ Joule Physics Laboratory, University of Salford, Manchester, United Kingdom \\ ${ }^{2}$ Photonics Laboratory, Tampere University of Technology, Finland \\ ${ }^{3}$ Department of Engineering, University of Roma 'Roma Tre', Italy \\ ${ }^{4}$ Autonomous University of State Morelos (UAEM), CIICAp, IICBA, Cuernavaca, 62209, Morelos, \\ Mexico \\ ${ }^{5}$ Laboratoire Interdisciplinaire Carnot de Bourgogne, UMR6303 CNRS/Université Bourgogne Franche- \\ Comté, Dijon, France \\ ${ }^{6}$ Original Perspectives Ltd, Tewkesbury, United Kingdom \\ ${ }^{7}$ Aalto University, School of Electrical Engineering, PO Box 13000, FI-00076 Aalto, Finland \\ 8 “Nanooptomechanics" Laboratory, ITMO University, St. Petersburg, 197101, Russia \\ ${ }^{9}$ Taras Shevchenko National University of Kyiv, Kyiv, Ukraine \\ ${ }^{10}$ Nazarbayev University, School of Science and Technology, Astana, Kazakhstan \\ E-mail: a.d.boardman@salford.ac.uk
}

Received 30 January 2017, revised 16 March 2017

Accepted for publication 17 March 2017

Published 9 October 2017

\begin{abstract}
The topics here deal with some current progress in electromagnetic wave propagation in a family of substances known as metamaterials. To begin with, it is discussed how a pulse can develop a leading edge that steepens and it is emphasised that such self-steepening is an important inclusion within a metamaterial environment together with Raman scattering and third-order dispersion whenever very short pulses are being investigated. It is emphasised that the self-steepening parameter is highly metamaterial-driven compared to Raman scattering, which is associated with a coefficient of the same form whether a normal positive phase, or a metamaterial waveguide is the vehicle for any soliton propagation. It is also shown that the influence of magnetooptics provides a beautiful and important control mechanism for metamaterial devices and that, in the future, this feature will have a significant impact upon the design of data control systems for optical computing. A major objective is fulfiled by the investigations of the fascinating properties of hyperbolic media that exhibit asymmetry of supported modes due to the tilt of optical axes. This is a topic that really merits elaboration because structural and optical asymmetry in optical components that end up manipulating electromagnetic waves is now the foundation of how to operate some of the most successful devices in photonics and electronics. It is pointed out, in this context, that graphene is one of the most famous plasmonic media with very low losses. It is a two-dimensional material that makes the implementation of an effectivemedium approximation more feasible. Nonlinear non-stationary diffraction in active planar anisotropic hyperbolic metamaterials is discussed in detail and two approaches are compared. One of them is based on the averaging over a unit cell, while the other one does not include sort of averaging. The formation and propagation of optical spatial solitons in hyperbolic metamaterials is also considered with a model of the response of hyperbolic metamaterials in terms of the homogenisation ('effective medium') approach. The model has a macroscopic dielectric tensor encompassing at least one negative eigenvalue. It is shown that light propagating in the presence of hyperbolic dispersion undergoes negative (anomalous) diffraction. The theory is ten broadened out to include the influence of the orientation of the optical axis with respect to the propagation wave vector. Optical rogue waves
\end{abstract}

11 Author to whom any correspondence should be addressed. 
are discussed in terms of how they are influenced, but not suppressed, by a metamaterial background. It is strongly discussed that metamaterials and optical rogue waves have both been making headlines in recent years and that they are, separately, large areas of research to study. A brief background of the inevitable linkage of them is considered and important new possibilities are discussed. After this background is revealed some new rogue wave configurations combining the two areas are presented alongside a discussion of the way forward for the future.

Keywords: waves, rogues, solitons

(Some figures may appear in colour only in the online journal)

\section{Introduction}

The discussions presented here deal with some current progress in electromagnetic wave propagation in a family of substances known as metamaterials [1-6]. The latter constitute a global revolution in science, which is widely acknowledged to have been launched properly in 1999, when Rodger Walser [1], from the University of Texas, published the word 'metamaterial'. The latter describes an artificial material that exhibits properties deriving from its composition, as opposed to its fundamental intrinsic physical property. This means, in the visible light domain, for example, that the size of the intrinsic components of metamaterials must be well below the excitation wavelength of operation. Hence, this implies the need to build nanostructures for the optical domain. The metamaterial family is growing, nicely, but only two important members will be discussed here, and they are called double negative and hyperbolic [7], respectively. The double negative metamaterial family members are isotropic and derive their name from the fact that they possess both negative permittivity and negative permeability. The hyperbolic members have uniaxial symmetry and, for an optic axis pointing along $z$, which is a popular wave propagation direction, can have a permittivity tensor that is diagonal, with positive $x$ and $y$ components and a negative $z$ component. In fact, there is a lot of versatility for hyperbolic media that helps to identify applications with type I hyperbolic metamaterial possessing just one negative component of the dielectric tensor negative. In the same hyperbolic family, there is type II that has two negative tensor components.

For this focus article, section 2 contains basic steps leading to a version of the widely known [7] nonlinear Schrödinger equation (NLSE). It is set up to lead to computer simulation of the behaviour of the double negative member of the metamaterial family. Since the type of NLSE produced here is dispersion-based it is capable of generating nonlinear waves called temporal solitons $[8,9]$ in such metamaterials. As discussed, in detail, later on, soliton means a nonlinear wave, in the form of a pulse, or a beam, in which broadening due to dispersion, or diffraction, is balanced by the presence of nonlinear amplitudes. Hence, the temporal soliton $[8,9]$ family member is a pulse in which broadening due to linear dispersion is balanced by the nonlinearity. It is selected here, without loss of generality, because it can form the basis of a delightful discussion of what have become known as rogue waves [10]. These can be created by a special input condition for the NLSE, consisting of a constant background being disturbed by small periodic perturbations. It is interesting to note, at this stage, that this kind of input can actually be a Peregrine soliton. The latter is named after Howell Peregrine [11], who, back in 1983, at the University of Bristol, looked brilliantly at these waves and found that they appear to come out of nowhere and then disappear quite suddenly again. Notwithstanding the pioneering work by Peregrine, his solitons are now broadly known as rogue waves. They still have a fascinating background in hydrodynamics, concerning waves leaping suddenly out of what seems to be a calm sea. They have attracted the name 'rogue waves' because they do appear from, apparently, nowhere, rise up to a dangerous height, potentially do damage to shipping, and then disappear from sight, without a trace.

In addition to the discussion of rogue waves other sections of this article analyse waves in hyperbolic metamaterials, with a detailed investigation of nonlinear stationary, or non-stationary, diffraction in active planar hyperbolic media.

As indicated up to now, the nonlinear metamaterial work that is emerging globally builds, credibly, upon the fact that solitons $[8,9]$ already have well-known roots in hydrodynamics dating back to John Scott Russell in 1834. It is interesting that this early work was not appreciated for some considerable time, yet, like the metamaterial world, it was also a global revolution. This means that the ability of new, artificial, substances, like metamaterials, to sustain soliton propagation is, therefore, a beautiful modern development. For a realistic discussion, it is convenient to focus upon only certain members of both the large soliton family and the growing metamaterial family. For this reason, without loss of generality, the attention of the next sections will be upon temporal solitons and double negative and hyperbolic metamaterials. The pulse-based basic physics of stable nonlinear excitations is that they rely upon balancing phase changes across their width that arise from the material dispersion and nonlinearity. They are stable if they are described by what is known as the one-dimensional cubic nonlinear $[8,9]$ Schrödinger equation (NLSE) briefly introduced earlier on. This fundamental one-dimensional NLSE is a completely satisfactory model to describe how electromagnetic pulses behave in a bounded dielectric, but any important additions to this basic equation have to be investigated as well and leads us towards the appreciation of the self-steepenng of pulses, for example.

Investigating solitons in a nonlinear double negative metamaterial does give rise to a generalised NLSE, which stresses that, by using the dispersion brought in by the relative dielectric permittivity and the relative permeability, a lot of new features emerge. For example, it soon becomes apparent that the sign of the self-steepening [12] parameter can be changed through frequency management. This has a dramatic 
impact upon modulation instability (MI) and short pulse propagation [13-16]. However, the work that will now unfold addresses how dispersion and metamaterial properties emerge as an influence upon the coefficients of the NLSE. It is, initially, surprising that the development below is in the absence of any modelling of the loss that solitons will encounter in a real system. This is because, as recent literature shows, it is possible to minimise the role of loss, and still introduce the fundamental double negative behaviour that is such a globally attractive feature of negative phase metamaterials. At this stage, it is also worth re-emphasising that there is a perfect analogy between the general, multidiscipline, nonlinear electromagnetic Schrödinger work and the study of unstable waves on deep oceans. It is not a surprise, then, that such instability in the hydrodynamic area was originally met with considerable, and broadly-based, scepticism. However, now it has been shown to be correct that the conditions for MI are the conditions for the creation of solitons. This applies very accurately to temporal solitons.

Since a double negative metamaterial exhibits an effective relative permittivity and an effective relative permeability, both of which are frequency dependent, an interrogating electromagnetic wave must have a wavelength that perceives only these effective properties because the metaparticles making up the material are sub-wavelength. Double negative metamaterials can have negative permittivity and negative permeability, both of which can be significantly frequency dependent. Here, it is assumed that such material is transparent and the aim, in section 2 is to determine just how the metamaterial properties can be associated with new soliton behaviour and to determine the extent to which this behaviour can be controlled. Two forms of the latter will be selected, one of which is magnetooptic control [17-21], and the other invokes nonlinearity.

Many materials can be operated in a nonlinear regime and this can be accessible through the use of pulses, that have sufficient power to drive a nonlinear polarisation addition to the linear displacement vector. In nonlinear optics, many different types of materials have been investigated and, broadly speaking, a lot of interest has been focused upon materials that yield so-called second-order nonlinearity, or a third-order response. Significant applications have emerged for materials that possess a centre of symmetry. For such media, it is the third-order nonlinearity that is important. This is the case for silica glass from which optical fibres are made. Almost paradoxically, a third-order nonlinearity associated with an isotropic material with a centre of symmetry leads to phenomena at the same frequency as that of the excitation, with the third-harmonic being ruled out because of a lack of phase matching. In this paper, it is a double-negative metamaterial with the classic third-order response that will be considered.

This provides an opportunity to investigate temporal solitons of the kind that has been written about so extensively for optical fibres. In the model adopted here, a planar guide is used, however, and the nonlinearity can arise from both dielectric and magnetic polarisation properties. The type of excitation selected actually comes from the large family of solitons and a temporal soliton is controlled by dispersion and nonlinearity. Strictly speaking, such a soliton emerges when the chirping induced by the dispersion and the nonlinearity exactly cancel each other out to give a constant phase across the pulse. The manner in which attention can be directed only towards nonlinearity and dispersion depends upon the waveguide structure that is supporting the solitons. For example, in an optical fibre, diffraction is eliminated in all directions perpendicular to the propagation axis, but a planar waveguide can also be used.

It is important to gain a thorough understanding of how using a metamaterial can impact upon phenomena such as self-steepening. For the latter, the dependence of the group velocity upon the intensity of the pulse causes the pulse to change shape. For a normal positive phase medium, the trailing edge of the pulse will steepen, yet propagating a pulse in a metamaterial can result in the steepening of the leading edge of the pulse. Very short pulses are associated with selfsteepening and they can suffer from Raman scattering. Also, it is possible that third-order group velocity dispersion can come into play and the introduction of magnetooptics can be a very important method of control.

\section{Temporal solitons in metamaterial waveguides}

\subsection{Nonlinear Schrödinger equation (NLSE)}

The standard NLSE often needs to be modified. For example, a new term that measures the self-steepening of the pulse may be required. Also, if a pulse is short in the time domain, it will possess a broad frequency spectrum and the design of the metamaterial may invoke Raman scattering. Basically, it is possible for high frequency components of a pulse to be converted to low-frequency components (Stokes lines), and it is also possible for the opposite to occur (anti-Stokes lines). Stokes lines are generated from the ground state and are more densely populated than anti-Stokes lines. If the pulses propagate in a metamaterial, then it is interesting to see what is the actual influence of the model artificial material being used upon self-steepening and to see whether the frequency dependence of the relative permittivity and the relative permeability can produce a significant outcome. This frequency dependence can be introduced through a variety of Drude models of the kind adopted here.

The lowest order temporal soliton is one which arises when the frequency chirp due to the nonlinearity is balanced by the chirp created by dispersion. Both the nonlinearity and the dispersion, therefore, add a chirp to the pulse, but as the two oppose each other, they can cancel each other out. Temporal soliton propagation is usually investigated using optical fibres and clearly, because of the guiding by the fibre core, diffraction is eliminated in both directions perpendicular to the propagation axis. Another option, however, is to excite the pulse in a planar waveguide. Here, the guided modes, propagating along the $z$-axis, have the diffraction in the $y$ direction eliminated, and it is assumed that pulses are sufficiently broad in the $x$-direction to also avoid diffraction. This 


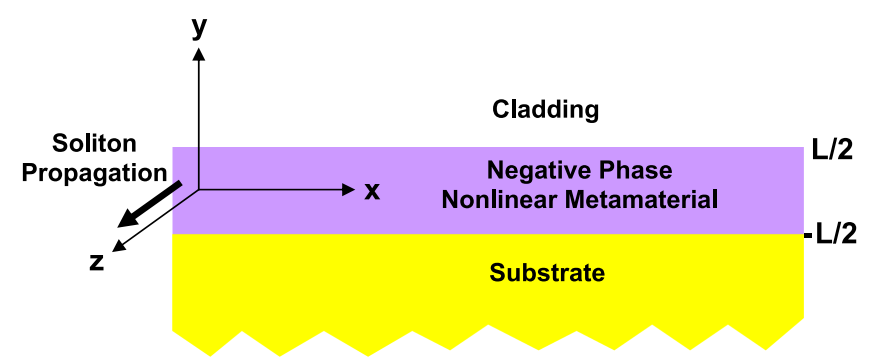

Figure 1 A metamaterial planar waveguide sustaining temporal solitons.

is the structure that is chosen here, and it is illustrated in figure 1 .

The global literature does provide a thorough description of temporal soliton propagation in, positive phase, optical fibres but the question is how does double negative, and, therefore, negative phase, metamaterial, affect the propagation characteristics of this type of soliton? MI is often the precursor to the study of pulse propagation in metamaterial structures and it has been the focus of a number of publications. In them it is clear that the familiar NLSE includes influences from second-order dispersion, third-order nonlinearity and possibly self-steepening. The latter arises because of a dependence of the group velocity upon the intensity of the pulse, and, in the absence of normal dispersion, will cause a pulse to change shape dramatically. In a positive phase medium, it is always the trailing edge of the pulse that will steepen. However, it has been shown that, for a pulse propagating in a metamaterial, there is a possibility for the leading edge of the pulse to steepen. In this context, magnetooptics can be introduced as a control mechanism.

For a positive phase medium, it is only electric nonlinearity that is included in any analysis, but there is a possibility for a metamaterial to possess double nonlinearity, where the origin of the nonlinearity is both electric and magnetic. This can be discussed in a regime for which loss is not specifically included. This is because loss-free, or sufficiently low-loss, metamaterials, are expected in the near future. Indeed, it is now worthwhile, therefore, to investigate outcomes based upon low loss rather than attenuation-dominated materials.

The route to the, standard, NLSE is to implement the slowly-varying amplitude approximation. This is when the electric and magnetic field is split into a fast variation, a linear modal field and an amplitude that varies slowly along the propagation axis. The standard NLSE then yields the slowly varying amplitude and accounts both for dispersion and nonlinearity. It is an adequate model of wave propagation events, in most cases. However, when pulses, whose duration is the order of a few femtoseconds, are under consideration, additional terms are needed in the NLSE in order to increase the accuracy of the predicted behaviour. One such term describes self-steepening of a pulse and is a vital nonlinear effect that has been briefly referred to above. Another possible addition is Raman scattering. As stated earlier, the latter either causes high frequency components of the pulse to be converted to low-frequency components, or vice versa. These are called Stokes lines and anti-Stokes lines, respectively. A further consideration is third-order dispersion. For a pulse propagating in a metamaterial, it is interesting to investigate whether the presence of a frequency-dependent relative permittivity and frequency-dependent relative permeability will have an influence on the higher-order terms.

A planar waveguide is used here because it has potential for integrated circuits. The $x$ and $y$ directions are guided and the temporal soliton propagates along the $z$-axis. Also the excitation is assumed to be so sufficiently wide in the $x$ direction that it minimises diffraction to such an extent that such diffraction can be discounted in the theoretical model. Hence, the derivation of the NLSE now emerges from a weakly guiding (quasi-bulk) scenario. Physically this is an appropriate approximation if $L$, the width of the metamaterial core, is large and the cladding and substrate are assumed to be total reflectors (metals). Another option is that the guiding structure should be arranged so that the refractive index of each layer is approximately matched across the boundaries. This option will be adopted here. If the index variation is restricted to be within $0.5 \%$ then, for a TM mode, the longitudinal component of the electric field, $E_{z}$ can be neglected because it is insignificant, when compared to the electric field component $E_{y}$. The guiding system will, therefore, have a semi-infinite cladding and substrate that, for the time being, is composed of standard, positive phase, dispersion-free dielectric. Selecting the substrate to be a magnetooptic material can lead to important control capabilities over temporal soliton behaviour. For the waveguide under consideration, only the TM mode is influenced by magnetooptics when the, transverse, Voigt configuration is used.

The metamaterial under consideration here, has a relative permittivity, and a relative permeability, that have a strong frequency dependence, and a frequency window exists in which both properties have a negative value. This double negative metamaterial is also assumed to be isotropic so that any complication arising from anisotropy is not necessary. Including anisotropy is just a complication that does not affect the principle outcomes. The pulse is set to be associated with an electric field $\mathbf{E}(\mathbf{r}, t)$, and a magnetic field $\mathbf{H}(\mathbf{r}, t)$, in the time domain, $t$, with $\mathbf{r}$ being the spatial vector $\mathbf{r}=(x, y, z)$, expressed in terms of the spatial Cartesian coordinates $x, y$ and $z$. For the double negative metamaterial, double nonlinearity means that both the relative permittivity and relative permeability are nonlinear. Even so, electrically nonlinear metamaterials, where only the permittivity is nonlinear have been discussed specifically in the literature, hence, an investigation into the propagation characteristics of these materials is an important global topic to study. In the Fourier domain, the electric and magnetic field associated with a temporal pulse, have the form

$$
\tilde{\mathbf{E}}(\boldsymbol{r}, \omega)=\int \mathbf{E}(\boldsymbol{r}, t) \mathrm{e}^{\mathrm{i} \omega t} \mathrm{~d} t, \quad \hat{\mathrm{H}}(\boldsymbol{r}, \omega)=\int \mathbf{H}(\boldsymbol{r}, t) \mathrm{e}^{\mathrm{i} \omega t} \mathrm{~d} t,
$$

where $\omega$ is an angular frequency, $\mathbf{r}$ is, again, simply the position vector, and $t$ is the time measured in the laboratory frame. The tilde used in (2.1) denotes a Fourier component. 
The basic electromagnetic equations, in the Fourier domain, can now be written as

$$
\begin{gathered}
\nabla \times \tilde{\mathbf{E}}(\mathbf{r}, \omega)=\mathrm{i} \omega \mu_{0} \mu_{L}(\omega) \hat{\mathbf{H}}(\mathbf{r}, \omega), \\
\nabla \times \tilde{\mathbf{H}}(\mathbf{r}, \omega)=-\mathrm{i} \omega \varepsilon_{0}\left(\varepsilon_{L}(\omega)+\chi^{(3)}|\tilde{\mathbf{E}}(\mathbf{r}, \omega)|^{2}\right) \\
\quad \times \tilde{\mathbf{E}}(\mathbf{r}, \omega)=-\mathrm{i} \omega \varepsilon_{0} \varepsilon(\omega) \tilde{\mathbf{E}}(\mathbf{r}, \omega),
\end{gathered}
$$

where $\varepsilon_{0}$ and $\mu_{0}$ are, as usual, the permittivity and permeability of free-space, respectively. For the third-order Kerr nonlinearity that has been introduced here, it is assumed that the coefficient, $\chi^{(3)}$, has the usual symmetry coefficient $3 / 4$ embedded within it. $\varepsilon_{L}(\omega)$ and $\mu_{L}(\omega)$ are the linear relative permittivity and linear relative permeability, respectively, of the isotropic metamaterial. The total permittivity is given by $\varepsilon(\omega)$ and includes both the linear and the nonlinear contributions.

From equations (2.2) and (2.3), the basic metamaterial Helmholtz equation is

$$
\nabla^{2} \mathbf{E}^{\%}+\frac{\omega^{2}}{c^{2}} \varepsilon(\omega) \mu_{L}(\omega) \mathbf{E}^{\%}=0 .
$$

This equation leads to the NLSE for temporal solitons. At this point, however, it is necessary to choose the type of propagating mode. The weakly guiding planar structure defined here can support either TE or TM modal fields. It will be the TM mode that is chosen, in preparation for the addition of a Voigt magnetooptic influence. This is because it is only the TM mode that is affected by this particular magnetooptic configuration, and the TE mode remains unaltered. The electric field components of the TM mode given here are $\left(\tilde{E}_{y}, \tilde{E}_{z}\right)$ but, for the weakly guiding structure discussed here, it is the propagation of the vector component $\tilde{E}_{y}$ that is of interest.

The NLSE is the result of taking a weakly nonlinear approximation. This approximation involves introducing a slowly-varying amplitude, $A(z, \omega)$, a complex linear modal field $F(y)$, which is totally characteristic of the specific guiding system, and a fast-variation involving the wave number $\beta_{0}$. This factorisation into three terms is expressed by

$$
\tilde{E}_{y}=A(z, \omega) F(y) \mathrm{e}^{\mathrm{i} \beta_{0} z} .
$$

The form of $\tilde{E}_{y}$ given in equation (2.5) can now be substituted into equation (2.4). A separation constant, i.e. an eigenvalue, $\tilde{\beta}$, can then be introduced, that leads to two equations of the form

$$
\begin{aligned}
& \frac{\partial^{2} F}{\partial y^{2}}+\frac{\omega^{2}}{c^{2}}\left(\varepsilon(\omega) \mu_{L}(\omega)-\tilde{\beta}^{2}\right) F=0, \\
& \frac{\partial^{2} A}{\partial z^{2}}+2 \mathrm{i} \beta_{0} \frac{\partial A}{\partial z}+\left(\tilde{\beta}^{2}-\beta_{0}^{2}\right) A=0 .
\end{aligned}
$$

Multiplying equation (2.6) by $A$, and (2.7) by $F$, and then recombining the equations to eliminate $\tilde{\beta}^{2}$ leads to

$$
\begin{aligned}
& F \frac{\partial^{2} A}{\partial z^{2}}+2 \mathrm{i} \beta_{0} F \frac{\partial A}{\partial z}-\beta_{0}^{2} F A=-A \frac{\partial^{2} F}{\partial y^{2}}-\frac{\omega^{2}}{c^{2}} \\
& \quad \times\left(\varepsilon(\omega) \mu_{L}(\omega)\right) F A .
\end{aligned}
$$

For a weakly guiding system, the second derivative of $F$ with respect to $y$ can be neglected on the grounds that it is, literally, very small, when compared with the other terms. From the layered guide structure illustrated in figure 1, the following distributions of the relative linear permittivity, $\varepsilon_{L}$, the relative linear permeability, $\mu_{L}$ and the nonlinear coefficient can be assigned.

$$
\begin{aligned}
& \varepsilon_{L}=\left\{\begin{array}{ll}
\varepsilon_{1}, & y \leqslant-\frac{L}{2}, \\
\varepsilon_{2}(\omega), & |y| \leqslant \frac{L}{2}, \\
\varepsilon_{3}, & y \geqslant \frac{L}{2},
\end{array} \quad \mu_{L}= \begin{cases}\mu_{L}=1, & y \leqslant-\frac{L}{2} \\
\mu_{2}(\omega), & |y| \leqslant \frac{L}{2} \\
\mu_{3}=1, & y \geqslant \frac{L}{2},\end{cases} \right. \\
& \chi^{(3)}= \begin{cases}0, & y \leqslant-\frac{L}{2}, \\
\chi^{(3)}, & |y| \leqslant \frac{L}{2} \\
0, & y \geqslant \frac{L}{2}\end{cases}
\end{aligned}
$$

The envelope equation for the entire weakly guiding waveguide system is therefore

$$
\frac{\partial^{2} A}{\partial z^{2}}+2 \mathbf{i} \beta_{0} \frac{\partial A}{\partial z}-\beta_{0}^{2} A+I_{1} A+I_{2}|A|^{2} A=0
$$

where

$$
\begin{aligned}
I_{1}= & \frac{\frac{\omega^{2}}{c^{2}} \int_{-\infty}^{\infty}|F|^{2} \varepsilon_{L}(\omega) \mu_{L}(\omega) \mathrm{d} y}{\int_{-\infty}^{\infty}|F|^{2} \mathrm{~d} y} \approx \frac{\omega^{2}}{c^{2}} \varepsilon_{L}(\omega) \\
& \times \mu_{L}(\omega) \frac{\int_{-\infty}^{\infty}|F|^{2} \mathrm{~d} y}{\int_{-\infty}^{\infty}|F|^{2} \mathrm{~d} y} \equiv \beta_{L}^{2}(\omega) .
\end{aligned}
$$

Note that because of the weakly guiding nature of the guide, the multiplication of $\varepsilon_{L} \mu_{L}$ in each of the layers is within $0.5 \%$ difference. Therefore, it can be assumed to not be a function of $y$, and can be moved outside the integration and the magnitude of the refractive index should be approximately the same in the core, as the cladding and substrate, but does not have to have the same sign. The basic envelope equation in the Fourier domain, up to this point, is, therefore,

$$
\begin{aligned}
& 2 \mathrm{i} \beta_{0} \frac{\partial A}{\partial z}+\frac{\partial^{2} A}{\partial z^{2}}+\left(\beta_{L}^{2}(\omega)-\beta_{0}^{2}\right) A+\frac{\omega^{2}}{c^{2}} \mu_{2}(\omega) \\
& \quad \times \bar{\chi}^{(3)}|A|^{2} A=0 .
\end{aligned}
$$

In practice, the temporal soliton contains a distribution of frequencies that are centred around an operational frequency, $\omega_{0}$. A Taylor expansion can be performed around this operational frequency, where the number of terms retained depends upon the application required. This expansion determines how (2.12) can be taken from the Fourier domain, to the time-domain. This transition to the time domain can be facilitated easily by re-writing the frequency, $\omega$ as $\left(\omega-\omega_{0}\right)+\omega_{0}$. Denoting the transformation to the timedomain by the symbol $\Rightarrow$ leads to the time-domain outcomes 
Laboratory frame

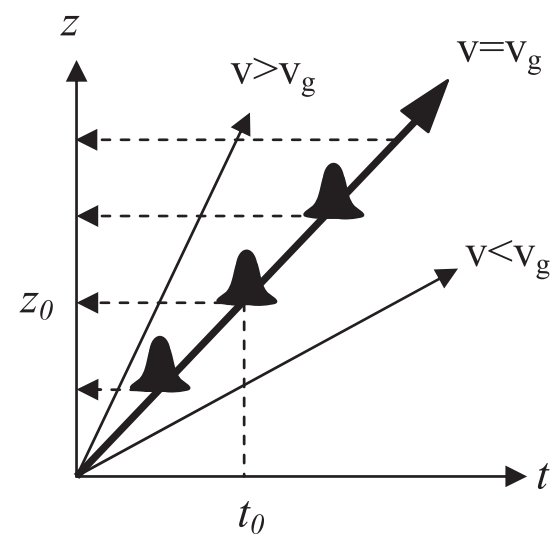

Global time
Frame moving at group velocity $\mathrm{v}_{\mathrm{g}}$

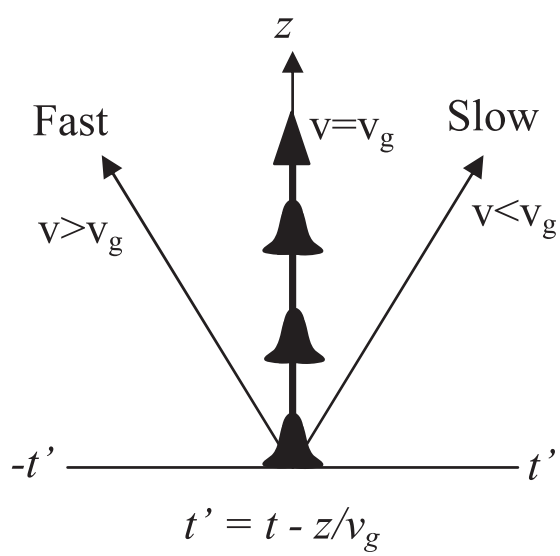

Local time

Figure 2. A sketch of the two frames of reference. (a) Illustrates a pulse in the laboratory frame, and (b) illustrates a pulse in the moving frame, that has a velocity $v_{g}$ relative to the laboratory frame.

$$
\begin{gathered}
\omega=\omega_{0}+\left(\omega-\omega_{0}\right) \Rightarrow \omega_{0}\left(1+\frac{\mathrm{i}}{\omega_{0}} \frac{\partial}{\partial t}\right), \\
\mu_{2}(\omega) \Rightarrow\left(\mu\left(\omega_{0}\right)+\left.\mathrm{i} \frac{\partial \mu}{\partial \omega}\right|_{\omega_{0}} \frac{\partial}{\partial t}\right) .
\end{gathered}
$$

Envelope equation, in the laboratory frame:

$$
\begin{aligned}
2 \mathrm{i} \beta_{0} & \frac{\partial A}{\partial z}+\frac{\partial^{2} A}{\partial z^{2}}+2 \mathrm{i} \beta_{0} \beta_{1} \frac{\partial A}{\partial z}-\left(\beta_{1}^{2}+\beta_{0} \beta_{2}\right) \frac{\partial^{2} A}{\partial t^{2}} \\
& -\mathrm{i}\left(\beta_{1} \beta_{2}+\frac{\beta_{0} \beta_{3}}{3}\right) \frac{\partial^{3} A}{\partial t^{3}}=-\frac{\omega_{0}^{2} \bar{\chi}^{(3)} \mu\left(\omega_{0}\right)}{c^{2}} \\
& \times\left(1+\frac{\mathrm{i}}{\omega_{0}} \frac{\partial}{\partial t}\right)^{2}\left(1+\left.\frac{\mathrm{i}}{\mu\left(\omega_{0}\right)} \frac{\partial \mu}{\partial \omega}\right|_{\omega_{0}} \frac{\partial}{\partial t}\right)|A|^{2} A .
\end{aligned}
$$

Here only terms up to the third derivative are retained and the origin of a self-steepening term can now be seen, along with third-order dispersion. Raman scattering is yet to appear. The next step is to transform this envelope equation to a moving frame that has a velocity $v_{g}$ relative to what will be called the laboratory frame. The merit of simulating the pulse in the moving frame is that the influence from short-pulse, or external, effects can be clearly observed, and this will become obvious in any computer simulations.

Figure 2 is intended to clarify the definition of moving and laboratory frames, and to illustrate show how a temporal soliton will propagate in them. Events in the laboratory frame are designated as being measured in global time, $t$. In the moving frame, the time, $t^{\prime}$ is defined as being local to the pulse. These two times are connected by the group velocity of the pulse, where the relationship is $t^{\prime}=t-z / v_{g}$. The zero point of time is the same in both frames, and as its position is arbitrary, it is useful to put the pulse centre at a time equal to zero. This means that the time will range from negative to positive values as the pulse is traversed. Figure 2 also shows the trajectories of pulses that are launched with a velocity greater, or less than, $v_{g}$, in both frames. They are labelled, in the moving frame, as fast, or slow, respectively.

The form of the NLSE appropriate for the propagation of very short pulses in standard materials, in both the laboratory and moving frame, can readily be found in the literature. The form being sought here will show how a metamaterial affects the self-steepening term.

The transformation from the laboratory frame, associated with the coordinates $(z, t)$, to a moving frame associated with coordinates $\left(z^{\prime}, t^{\prime}\right)$ can now be performed, using the arrows $(\rightarrow)$ to label the process, in the following manner

$$
\begin{aligned}
z \rightarrow & z^{\prime}, \quad t \rightarrow t^{\prime}-\beta_{1} z, \quad \beta_{1}=\left.\frac{\partial k}{\partial \omega}\right|_{\omega_{0}}=\frac{1}{V_{g}}, \\
\frac{\partial}{\partial t} \rightarrow & \frac{\partial}{\partial t^{\prime}} \quad \frac{\partial}{\partial z} \rightarrow \frac{\partial}{\partial z^{\prime}}-\beta_{1} \frac{\partial}{\partial t^{\prime}} \quad \frac{\partial^{2}}{\partial z^{2}} \rightarrow \frac{\partial^{2}}{\partial z^{\prime 2}} \\
& -2 \beta_{1} \frac{\partial}{\partial z^{\prime} \partial t^{\prime}}+\beta_{1}^{2} \frac{\partial^{2}}{\partial t^{\prime 2}} .
\end{aligned}
$$

After these transformations are applied, the main envelope equation becomes

$$
\begin{aligned}
& 2 i \beta_{0}\left(1+i \frac{\beta_{1}}{\beta_{0}} \frac{\partial}{\partial t^{\prime}}\right) \frac{\partial A}{\partial z^{\prime}}+\frac{\partial^{2} A}{\partial z^{\prime 2}}-\beta_{0} \beta_{2} \frac{\partial^{2} A}{\partial t^{\prime 2}} \\
& -i\left(\beta_{1} \beta_{2}+\frac{\beta_{0} \beta_{3}}{3}\right) \frac{\partial^{3} A}{\partial t^{\prime 3}}=-\frac{\omega_{0}^{2} \bar{\chi}^{(3)} \mu\left(\omega_{0}\right)}{c^{2}} \\
& \times\left(1+\frac{i}{\omega_{0}} \frac{\partial}{\partial t^{\prime}}\right)^{2}\left(1+\left.\frac{i}{\mu\left(\omega_{0}\right)} \frac{\partial \mu}{\partial \omega}\right|_{\omega_{0}} \frac{\partial}{\partial t^{\prime}}\right)|A|^{2} A .
\end{aligned}
$$

Using the slowly varying amplitude approximation, it is common practice to neglect the $\frac{\partial^{2}}{\partial z^{\prime 2}}$ term. However, now that 
higher-order terms have been retained, it is necessary to reexamine this conclusion. Broadly speaking, the following order of magnitude conclusions can be employed $\frac{\partial}{\partial z^{\prime}} \sim \frac{\partial^{2}}{\partial t^{\prime 2}} \sim$ $\chi^{(3)}|A|^{2}$ and therefore $\frac{\partial^{2}}{\partial t^{\prime} \partial z^{\prime}} \sim \frac{\partial^{3}}{\partial t^{\prime 3}}$. On this basis, the $\frac{\partial^{2}}{\partial z^{\prime 2}}$ term can still be safely neglected.

$$
\begin{aligned}
& \text { Multiplying equation (2.20) by }\left(1-\frac{\mathrm{i} \beta_{1}}{\beta_{0}} \frac{\partial}{\partial t^{\prime}}\right) \text { yields }
\end{aligned}
$$

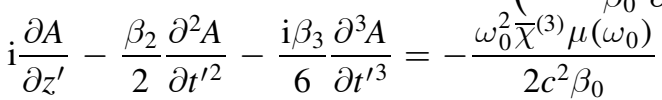

$$
\begin{aligned}
& \times\left(1+\frac{\mathrm{i}}{\omega_{0}} \frac{\partial}{\partial t^{\prime}}\right)^{2}\left(1-\frac{\mathrm{i} \beta_{1}}{\beta_{0}} \frac{\partial}{\partial t^{\prime}}\right)\left(1+\left.\frac{\mathrm{i}}{\mu\left(\omega_{0}\right)} \frac{\partial \mu}{\partial \omega}\right|_{\omega_{0}}\right. \\
& \left.\times \frac{\partial}{\partial t^{\prime}}\right)|A|^{2} A \text {. }
\end{aligned}
$$

This can be simplified to give

$$
\begin{aligned}
& \mathrm{i} \frac{\partial A}{\partial z^{\prime}}-\frac{\beta_{2}}{2} \frac{\partial^{2} A}{\partial t^{\prime 2}}-\frac{\mathrm{i} \beta_{3}}{6} \frac{\partial^{3} A}{\partial t^{\prime 3}}+\frac{\omega_{0}^{2} \bar{\chi}^{(3)} \mu\left(\omega_{0}\right)}{2 c^{2} \beta_{0}} \\
& \quad \times\left(|A|^{2} A+\mathrm{i} S \frac{\partial}{\partial t^{\prime}}\left(|A|^{2} A\right)\right)=0 .
\end{aligned}
$$

Equation (2.19) is now the final version of the dimensional Schrödinger equation, and includes the standard nonlinear, and self-steepening terms, along with both second- and thirdorder dispersion. The equation involves the self-steepening term $S=\left(1+\frac{\mathrm{i}}{\omega_{0}} \frac{\partial}{\partial t^{\prime}}\right)^{2}\left(1-\frac{\mathrm{i} \beta_{1}}{\beta_{0}} \frac{\partial}{\partial t^{\prime}}\right)\left(1+\left.\frac{\mathrm{i}}{\mu\left(\omega_{0}\right)} \frac{\partial \mu}{\partial \omega}\right|_{\omega_{0}} \frac{\partial}{\partial t^{\prime}}\right)$. After these operators are expanded, the coefficient of selfsteepening takes the form

$$
\begin{aligned}
S & =\frac{1}{\omega_{0}}\left[2-\frac{\beta_{1} \omega_{0}}{\beta_{0}}+\left(\frac{\omega}{\mu} \frac{\partial \mu}{\partial \omega}\right)_{\omega_{0}}\right] \\
& =\frac{1}{\omega_{0}}\left[2+\frac{\left|V_{p}\right|}{V_{g}}+\left(\frac{\omega}{\mu} \frac{\partial \mu}{\partial \omega}\right)_{\omega_{0}}\right] .
\end{aligned}
$$

In which it is recognised that the excitation is a backward wave for which the phase velocity is $v_{p}<0$ and the group velocity is $v_{g}>0$. The physical consequence of the term $\frac{\partial}{\partial t^{\prime}}\left(|A|^{2} A\right)$, is to steepen one edge of the pulse. This effect is documented well in the literature, but the influence that the metamaterial imposes is to change, radically, the form of the coefficient of self-steepening, $S$. The form of the self-steepening coefficient in a standard positive phase medium is simply $1 / \omega_{0}$. If the limit is taken, where no metamaterial properties are present, $S$ goes back to the standard form only for a dispersion-free medium with $v_{p}=v_{g}$.

Equation (2.19) is the dimensional form of the NLSE required. It can be cast into a dimensionless form through the following definitions

$$
z^{\prime}=L_{D} Z, \quad t^{\prime}=T_{0} T, \quad A=\psi / \sqrt{\frac{\omega_{0}^{2}}{2\left|\beta_{0}\right| c^{2}}\left|\mu\left(\omega_{0}\right)\right| \bar{\chi}^{(3)}},
$$

where the dispersion length is $L_{D}=\frac{T_{0}^{2}}{\left|\beta_{2}\right|}$ and $T_{0}$ is interpreted as the pulse width. At this point in the derivation it is appropriate to include Raman scattering and is introduced through the parameter $\tau_{R}$, defined below. The form of the dimensionless NLSE that includes self-steepening and Raman scattering is now

$$
\begin{aligned}
& \mathrm{i} \frac{\partial \psi}{\partial Z}-\frac{1}{2} \operatorname{sgn}\left(\beta_{2}\right) \frac{\partial^{2} \psi}{\partial T^{2}}-\mathrm{i} \delta_{3} \frac{\partial^{3} \psi}{\partial T^{3}}+\operatorname{sgn}\left(\bar{\chi}^{(3)}\right) \\
& \quad \times\left(|\psi|^{2} \psi+\mathrm{i} S \frac{\partial}{\partial T}\left(|\psi|^{2} \psi\right)-\tau_{R} \psi \frac{\partial}{\partial T}\left(|\psi|^{2}\right)\right)=0
\end{aligned}
$$

where the third-order dispersion is quantified by $\delta_{3}=$ $\frac{\beta_{3}}{6\left|\beta_{2}\right| T_{0}}$, the self-steepening parameter is $S=\frac{1}{\omega_{0} T_{0}}$ $\left[2-\frac{\beta_{1} \omega_{0}}{\beta_{0}}+\left(\frac{\omega}{\mu} \frac{\partial \mu}{\partial \omega}\right)_{\omega_{0}}+\left(\frac{\omega}{\chi^{(3)}} \frac{\partial \chi^{(3)}}{\partial \omega}\right)_{\omega_{0}}\right]$ and Raman scattering is represented by $\tau_{R}=\frac{\int_{-\infty}^{\infty} t R(t) \mathrm{d} t}{T_{0}}=\frac{T_{R}}{T_{0}}$, using a Raman response function $R(t)$. This equation exhibits a specific form for the self-steepening coefficient. The Raman scattering coefficient, $\tau_{R}=\frac{T_{R}}{T_{0}}$ is inversely proportional to the pulse duration and the form of $T_{R}$ can easily be found in the literature. A typical value for a nonlinear material is the order of $10^{-15} \mathrm{~s}$, or a femtosecond. This means that for a pulse with a duration of $21 \mathrm{fs}$, the Raman scattering coefficient would take a value of $\tau_{R} \approx 0.033$. Once again, it should be noted that this coefficient is independent of frequency and any metamaterial properties.

Equation (2.22), the dimensionless form of the NLSE, can be conveniently used to generate some illustrative computer simulations. In addition, it can then be used, in section 3, to generate rogue waves and their dependence upon the influence of metamaterials.

\subsection{Magnetooptic influence}

If the guide shown in figure 1 is modified, so that it contains some magnetooptic material, it will be possible to obtain control over the temporal solitons by the means of an externally applied magnetic field. Classically, there are three standard options for the type of magnetooptic control that can be achieved. Very elegantly, the orientation of the magnetic field with respect to the propagation direction leads to fascinating outcomes for the pulse behaviour. They are known as the Voigt, Faraday, and polar configurations. Here, it is the Voigt effect that is adopted. This selection does not induce any polarisation coupling and enables a very transparent form of control to be developed. In this configuration, the external magnetic field is applied in a direction that is perpendicular to the propagation direction: in this case, along the $X$-axis. Once again, it should be noted that the waveguide must be asymmetric, with the magnetooptic material only being included in either the cladding, or the substrate, for any effect to be present. The waveguide in question will be of the form given in figure 1, but with the substrate replaced with a magnetooptic material and the externally applied magnetic field being orientated along the $X$-direction.

External magnetic fields actually induce off-diagonal elements in the relative permittivity tensor of a magnetooptic 
material. The form of this tensor, appropriate for the Voigt configuration, with an external magnetic field located along the $X$-axis, is

$$
\varepsilon_{m}=\left(\begin{array}{ccc}
\left(n_{m}^{2}\right) & 0 & 0 \\
0 & \left(n_{m}^{2}\right) & \left(-\mathrm{i} Q n_{m}^{2}\right) \\
0 & \left(\mathrm{i} Q n_{m}^{2}\right) & \left(n_{m}^{2}\right)
\end{array}\right)=\left(\begin{array}{ccc}
\varepsilon & 0 & 0 \\
0 & \varepsilon & \varepsilon_{y z} \\
0 & -\varepsilon_{y z} & \varepsilon
\end{array}\right),
$$

where $n_{m}=\sqrt{\varepsilon}$ and is the refractive index of the magnetooptic material in the absence of a magnetic field, and $Q$ is the magnetooptic parameter specific to the material. For convenience, $\varepsilon_{y z}$ is also defined here as the off-diagonal permittivity element $\varepsilon_{y z}=\mathrm{i} n_{m}^{2} Q$. The value of $Q$ for standard magnetooptic materials is the order of $10^{-4}$, but modern magnetooptic materials have values that can be as large as the order of $10^{-2}$. Again, it is emphasised that it is only the TM mode that will be influenced by the magnetooptic effect: the TE mode remains unaltered.

The magnetooptic presence perturbs the slowly varying amplitude solution of the Schrödinger equation. The contribution to $\frac{\partial A}{\partial Z}$ can be developed for either a TE, or TM, polarised wave. The slowly varying amplitudes adopted are $A^{\mathrm{TE}}$, or $A$, respectively. The outcome is very simple in form, and for each polarisation, the rate of change of the slowly varying amplitude is

$$
\begin{aligned}
& \text { TE-polarised: } \mathrm{i} \frac{\partial A^{\mathrm{TE}}}{\partial z}=0, \\
& \text { TM-polarised: } \mathrm{i} \frac{\partial A}{\partial z}=\frac{\omega}{c}\left\langle\varepsilon_{y z}\right\rangle A,
\end{aligned}
$$

where $\left\langle\varepsilon_{y z}\right\rangle$ is the effective averaged magnetooptic coefficient over the waveguide structure. It is confirmed in equation (2.24), that it is only the TM mode that is affected by this form of magnetooptics. $\left\langle\varepsilon_{y z}\right\rangle$ contains an average over the modal field of the waveguide structure, and an integration along $y$ is involved. Clearly, this will be zero if the waveguide structure is symmetric. However, if the structure is asymmetric, it is possible to create a Voigt effect of order $Q$.

The perturbative method used to arrive at equation (2.22) allows this term to simply be added to the NLSE using $G \psi$ to account for all of the nonlinear terms. Therefore, the NLSE, in the presence of magnetooptics and in the moving frame of the pulse, is

$$
\mathrm{i} \frac{\partial \psi}{\partial Z}-\frac{1}{2} \operatorname{sgn}\left(\beta_{2}\right) \frac{\partial^{2} \psi}{\partial T^{2}}-\mathrm{i} \delta_{3} \frac{\partial^{3} \psi}{\partial T^{3}}+v \psi=G \psi
$$

The normalisations used to arrive at (2.25) give a magnetooptic coefficient of the form $v=\frac{\omega}{c} L_{D} n_{m}^{2}\langle Q\rangle$, where $\langle Q\rangle$ is the effective magnetooptic parameter over the waveguide structure.

If $v$ is chosen to be simply a constant, the only outcome would be to impart a phase shift to the soliton. In order to create a more significant effect, $v$ must be time-dependent. Considering this type of time-dependence creates a need to embrace the different frames of reference. The pulse is generally viewed in the moving frame, whereas the external magnetic field is clearly in the laboratory frame. This means that equation (2.25) needs to be considered carefully to ensure that the correct function is allocated to the magnetic field distribution. As equation (2.25) refers to the moving frame of the pulse, a transformation must also be applied to the magnetic field function to transform it to the moving frame before it is added to the final equation. This transformation involves the group velocity, $v_{g}$, of the pulse. Therefore, the magnetic field function, when expressed in the moving frame, and also in dimensionless coordinates, is of the form

$$
\begin{aligned}
v\left(T_{L}\right) & =v\left(T+\frac{Z}{V_{g}}\right)=\frac{\omega}{c} L_{D} n_{m}^{2} Q_{\text {sat }} f\left(T+\frac{Z}{V_{g}}\right) \\
& =v_{\max } f\left(T+\frac{Z}{V_{g}}\right),
\end{aligned}
$$

where $T_{L}$ is the dimensionless time in the laboratory frame, and is normalised via the equation $t=T_{0} T_{L} \cdot f$ is a function that describes the magnetic field dependence on time, and involves a maximum value $v_{\max }$. How the introduction of this type of function will change the propagation characteristics of a soliton can be shown in the simulations.

2.2.1. Simulation of soliton behaviour. The NLSE, complete with the short-pulse and magnetooptic terms, will yield, numerically, a number of great scenarios. The terms in the NLSE introduce metamaterial influences upon the soliton behaviour. The simulations will show how the effects can be combined to give interesting conclusions. These impacts can also be addressed in a discussion of rogue waves, through special input conditions.

Because the coefficient of self-steepening for a metamaterial is strongly frequency dependent, it can be either greater, or less, than zero. The effect of this metamaterial-driven outcome on soliton behaviour can then be readily determined with carefully selected simulations. In addition, although, Raman scattering is not metamaterial dependent, for short pulses where the self-steepening coefficient is significant, the Raman term will also be important, so this also needs investigation.

Simulations can be performed that show how all of these effects combine to give metamaterial-specific outcomes, and also how they can be controlled by a magnetooptic environment. For the magnetooptic term to have an effect, other than a change to the phase of the soliton, the external magnetic field must be a function of time. Hence, the waveguide can be demagnetised at different times, with respect to the pulse creation. This can all be described by the function below, where $t_{v}$ is the parameter being altered.

$$
v=\frac{v_{\max }}{2}\left(1-\tanh \left(\frac{t-t_{v}+Z / V_{g}}{\Delta t}\right)\right) .
$$

It is imperative to include the group velocity into this function, otherwise the outcome would be a change in amplitude, rather than a time-shift of the pulse.

All-optical computing will require the ability to manipulate pulses into switching formats. It can be seen in 


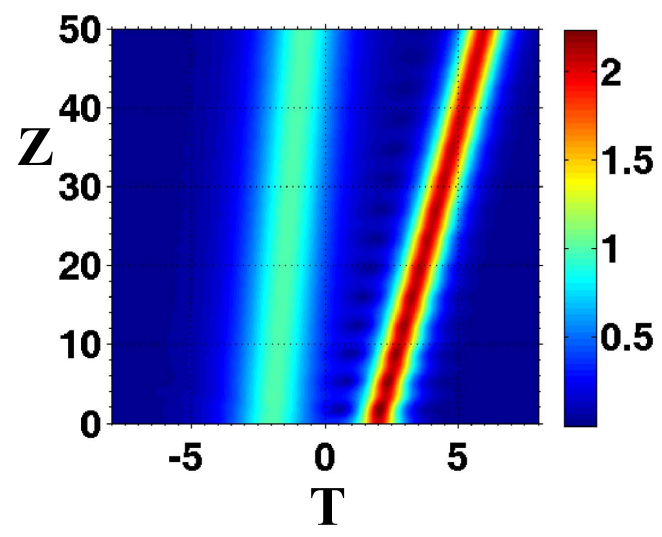

(a)

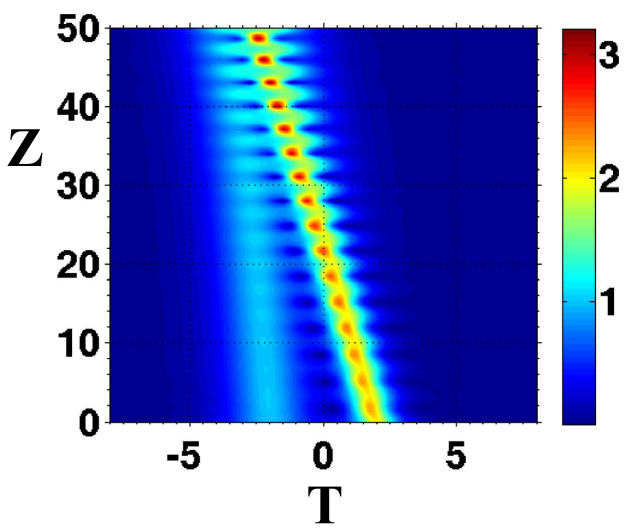

(b)

Figure 3. Inputs: $\psi=\operatorname{sech}(T+2)+2 \operatorname{sech}(2(T-2))$. (a) $S=0.02$, and (b) $S=-0.02$.

figure 3(a), that in the positive self-steepening case, the pulses have a reduced velocity and therefore arrive at the output plane $(Z=50)$ at a later time than expected, and in the negative self-steepening case the two pulses have an increased velocity, and arrive at the output plane at an earlier time than expected. It can also be seen that in both cases of the two different pulses, it is the shorter pulse that is more strongly affected by the self-steepening. Figure 3(b) shows an interesting interaction, causing a cross-over of the arrival times at the receiving port. This is a very impressive outcome arising from the use of a metamaterial.

The NLSE, derived above, provides an excellent model for pulse behaviour in metamaterial guides. The type of nonlinearity used here is assumed to be Kerr-like. It is emphasised that self-steepening is an important inclusion, but that other effects, such as Raman scattering and third-order dispersion should also be considered when very short pulses are being investigated. It is emphasised that the selfsteepening coefficient is highly metamaterial-driven, and the form derived here is consistent with the literature. The coefficient of Raman scattering is immune to the frequency dependence of the metamaterial, and is of the same form whether a normal positive phase, or a metamaterial waveguide is the vehicle for soliton propagation.

Finally, as can be seen in figure 4, there is an attractive interplay between self-steepening and magnetooptics so the influence of magnetooptics provides a beautiful and important control mechanism, that alters the speed of a pulse. It is anticipated that this work will have a significant impact upon the design of data control systems for optical computing.

\section{Asymmetric hyperbolic metamaterials}

\subsection{Asymmetry in EM devices and hyperbolic media with graphene}

The objective of this section is to examine interesting properties of hyperbolic media which exhibit asymmetry of supported modes due to the tilt of optical axes. Such a topic deserves elaboration since structural and optical asymmetries in components manipulating electromagnetic waves is a feature characterising the operation of some of the most successful devices in photonics and electronics during the last decades. From asymmetric sub-wavelength unit cells of microwave transmission lines which make exotic and controllable metamaterials [22], to photonic crystals employing asymmetries to steer and accumulate light [23], structures supporting unequal modes of processing the flow of electromagnetic energy is of prime usefulness. Ultra-effective asymmetric light-trapping photovoltaic devices have been designed [24], while it has been recently shown [25] that by introducing tailored optical asymmetry in clusters of particles manipulating light, efficient beam guiding is achieved, controllable by the operational frequency. Rudimentary designs like split ring resonators [26] led to backward-wave effective media with numerous applications are working with a structural asymmetry creating the necessary sharp resonances. Even the concept of non-reciprocity which is utilised in any signal manipulation except for filtering, is based on emulating asymmetries in the macroscopic description [27] mainly through external bias in a variety of oscillating wavelengths from mid-infrared [28], to radio [29] and acoustical [30].

Hyperbolic media [31,32] which are uniaxial materials behaving as ordinary dielectrics along one direction and as metals along the other, constitute a preferential class of media in building wave-manipulating structures with asymmetries. Such materials have borrowed their name from the shape of their dispersion curve which is a hyperbola [33]. The basic difference between hyperbola and ellipse (ordinary uniaxial dielectric dispersion relation) is that the former extends to infinity; therefore, the hyperbolic material can support propagating wavenumbers with infinite magnitude. Such a shrink in the effective wavelength can offer unique packaging opportunities in optical devices, infinite density of states [34] and lead to designs of ultra-efficient absorbers [35], radiation enhancers [36-38], nano-resonators [39], diffraction-free focusing and imaging [40]. The most direct way to fabricate a structure emulating hyperbolic media properties is to stack parallel layers or sheets of plasmonic materials or even perfect metals [41] into an ordinary host of positive permittivity. If that lattice is very densely populated and deeply subwavelength, then effective-medium approximation [42, 43] is 


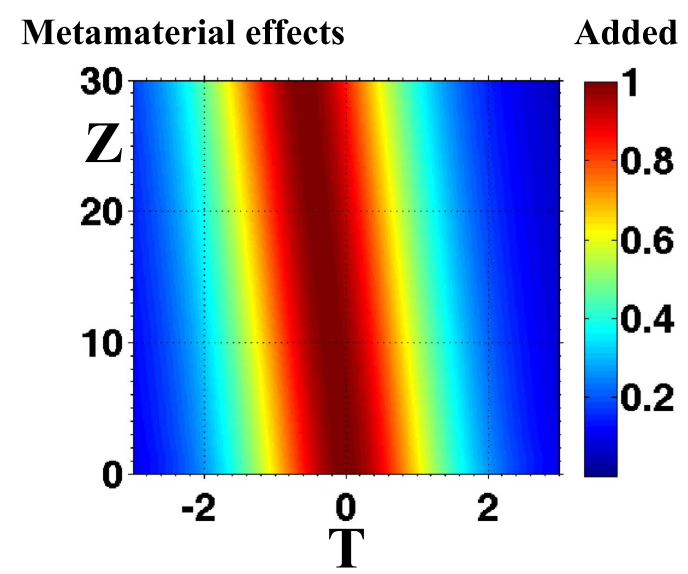

(a)
Added magnetooptic control

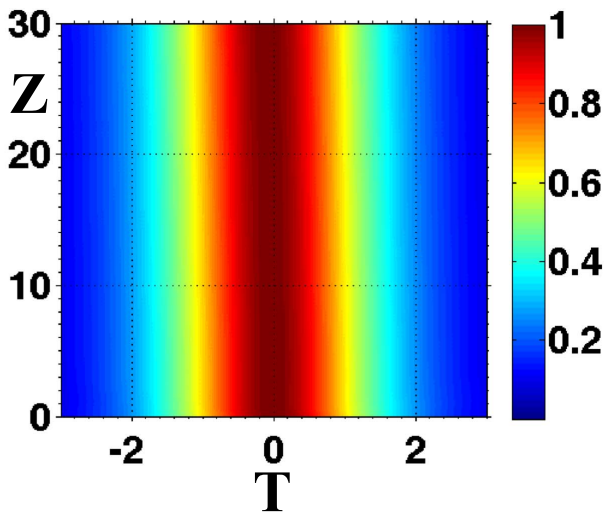

(b)

Figure 4. Example of the interplay between self-steepening and magnetooptic control. $\psi=\operatorname{sech}(T)$. (a) $S=-0.02, v_{\max }=0$, (b) $S=-0.02, v_{\max }=-0.5$.

applicable: the permittivity along the direction parallel to the layers would be close to that of the host, namely positive and the permittivity along its normal axis would be plasmonic.

One of the most famous plasmonic media with very low losses is graphene [44] which additionally possesses a number of advantages. First of all, it is a two-dimensional material which offers wave manipulation infinitely concentrated in space (oneatom-thick) and makes the implementation of effective-medium approximation more feasible. Furthermore, the properties [45] of graphene hardly resemble the ones of the bulk graphite from which it has been exfoliated; most characteristically, it has very high surface conductivity [46] which admits the process of waves passing through it. A significant advantage of graphene is its extreme durability and flexibility which accompanies its characteristic of being easily isolated in stable form. It has been reported [47] that spatially inhomogeneous and non-uniform conductivity patterns across a flake of graphene, can produce an one-atom-thick platform for infrared metamaterials and transformation optics devices. Graphene is also very commonly used to inherit exotic effective properties into common backgrounds like epsilon-near-zero response [48] or hyperbolic dispersion [49]; therefore, graphene-based asymmetric hyperbolic media would be the major objective of our research.

As indicated above, asymmetry is in the nature of hyperbolic medium due to the totally different permittivity along its two major axes; however, if we are interested in asymmetry of excited modes, we should tilt the optical axes. The new effective properties appearing in a hyperbolic slab due to the asymmetry induced when arbitrarily tilting the optical axis are analysed in [50] and the asymmetric modes supported by such a structure are obtained in [51]. In such asymmetric hyperbolic structures, interesting phenomena have been identified giving rise to applications like superPlanckian far-zone thermal emission [52] and nonlinear signal manipulation employing backward-propagating waves [53-55]. Furthermore, similar hyperbolic layers are proven to exhibit extremely efficient attenuation [56] and absorption performance for specific incidence angles [57], which is wideband [58] and realistic in terms of construction [59].
In this section, we are initially considering an arbitrary uniaxial medium with tilted optical axis and determining the propagation constants of the permitted waves into its volume. We find the condition for maximum asymmetry between the modes and we realise that is fulfilled only for hyperbolic media (with suitable tilt). Most importantly, the propagation constants differ dramatically each other and, in the lossless limit, their difference become infinite (one finite, one infinite). For a certain class of hyperbolic permittivities, the aforementioned extreme asymmetry can be combined with perfect impedance matching with free-space leading to zero reflections for a specific angle of incidence. We propose a realistic design of suitable doped, free-standing graphene multilayers and determine the period of the lattice and the characteristics of graphene to possess the aforementioned combination of unique features (extreme asymmetry and perfect matching) for specific bands. For these values of input parameters, we examine a finite-thickness slab of such a tilted graphene multilayered structure in terms of its absorbing efficiency. We realise that both wideband and wide-angle performance is achieved and huge asymmetry between the modes is recorded in the vicinity of Lorentz resonances. To this end, we regard the corresponding waveguiding structure which has been additionally grounded. The waveguidance condition is derived and the dispersion curves are sketched revealing the existence of: backward propagating, forward propagating and evanescent modes into the slab depending on the operational frequency.

\subsection{Asymmetry of modes in uniaxial media}

We consider a uniaxial medium characterised by two relative permittivities: $\varepsilon_{\|}$and $\varepsilon_{\perp}$ while being magnetically inert $\left(\mu=\mu_{0}\right)$. We use the Cartesian coordinate system $(x, y, z)$ defined at figure 5, where a slab of thickness $h$ of that material is shown; the optical axis of the medium forms a tilt angle $\theta$ with the $z$ axis (normal to the interfaces). The incident plane wave propagates into free space and possesses a sole $y$ magnetic component (TM wave), so that electric field lies on 


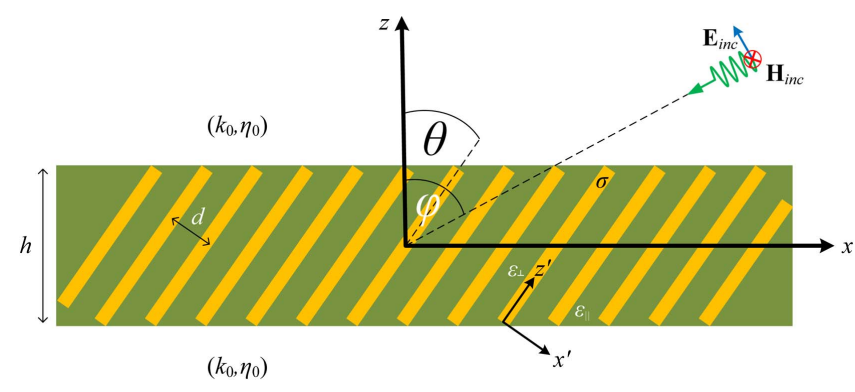

Figure 5. The schematic of our structure. A uniaxial $\left(\varepsilon_{\|}, \varepsilon_{\perp}\right)$ slab of thickness $h$ and tilted optical axis by angle $\theta$, gets excited by a TM obliquely incident plane wave along direction forming an angle $\phi$ with the normal axis. The uniaxial permittivities may be the macroscopic averaging of the response from graphene multilayers with surface conductivity $\sigma$.

the $z x$ plane and both permittivities get activated; furthermore, its direction of propagation makes an angle $\phi$ with the $z$ axis of our Cartesian coordinate system (oblique incidence). For $\theta=0$, the permittivity tensor of the medium is diagonal and for $\theta \neq 0$ is full as shown below:

$$
\begin{aligned}
{[\varepsilon(\theta=0)] } & =\left[\begin{array}{ccc}
\varepsilon_{\|} & 0 & 0 \\
0 & \varepsilon_{\perp} & 0 \\
0 & 0 & \varepsilon_{\perp}
\end{array}\right], \\
{[\varepsilon] } & =\left[\begin{array}{ccc}
\varepsilon_{x x} & 0 & \varepsilon_{z x} \\
0 & \varepsilon_{\perp} & 0 \\
\varepsilon_{x z} & 0 & \varepsilon_{z z}
\end{array}\right],
\end{aligned}
$$

where: $\quad \varepsilon_{x z}=\varepsilon_{z x}=\left(\varepsilon_{\|}-\varepsilon_{\perp}\right) \quad \cos \theta \quad \sin \theta, \quad \varepsilon_{x x}=\varepsilon_{\perp}$ $\sin ^{2} \theta+\varepsilon_{\|} \cos ^{2} \theta$ and $\varepsilon_{z z}=\varepsilon_{\perp} \cos ^{2} \theta+\varepsilon_{\|} \sin ^{2} \theta$. The time dependence of type exp $(-\mathrm{i} \omega t)$ with circular frequency $\omega$ is suppressed throughout the analysis. The tilt angle is kept within the interval: $0<\theta<90^{\circ}$, while the incidence angle covers all the directions across the upper half space: $90^{\circ}<\phi<90^{\circ}$.

If we seek for waves with magnetic field $\mathbf{H}=$ $\hat{\mathbf{y}} \exp \left(-\mathrm{i} k_{x} x-\mathrm{i} k_{z} z\right)$ into the aforementioned medium and take into account that $k_{x}=k_{0} \sin \phi$, as imposed by the incident illumination $\left(k_{0}=\omega \sqrt{\varepsilon_{0} \mu_{0}}\right.$ is the free-space wavenumber), we obtain the following two possible values (superscripts 1 and 2) for the propagation constant $k_{z}$ :

$k_{z}^{(1,2)} / k_{0}$

$=\frac{\left(\varepsilon_{\perp}-\varepsilon_{\|}\right) \sin (2 \theta) \sin \phi \mp \sqrt{2} \sqrt{\varepsilon_{\perp} \varepsilon_{\|}\left[\left(\varepsilon_{\perp}+\varepsilon_{\|}\right)+\left(\varepsilon_{\perp}-\varepsilon_{\|}\right) \cos (2 \theta)-2 \sin ^{2} \phi\right]}}{\varepsilon_{\perp}+\varepsilon_{\|}+\left(\varepsilon_{\perp}-\varepsilon_{\|}\right) \cos (2 \theta)}$.

Our objective is to examine the conditions under which the two waves with propagation constants (3.2), supported by the tilted uniaxial medium are as different as possible each other (maximal asymmetry). For the sake of brevity and without loss of generality, we can consider that one of the two permittivities of our material is equal to that of vacuum: $\varepsilon_{\|}=1$. Furthermore, we are initially regarding lossless media $\left(\varepsilon_{\perp} \in \mathbb{R}\right)$.

By inspection of (3.2), one clearly sees that the common denominator gets nullified when the tilt angle takes the following special value $\theta_{0}$ :

$$
\theta=\theta_{0}=\frac{1}{2} \arccos \left(\frac{1+\varepsilon_{\perp}}{1-\varepsilon_{\perp}}\right),
$$

which is real only if $\varepsilon_{\perp}$ is negative. Indeed, for a fixed angle $\theta$, the value of the transversal permittivity which makes the denominator of (3.2) vanish is: $\varepsilon_{\perp}=\frac{\cos (2 \theta)-1}{\cos (2 \theta)+1}<0$. Such a conclusion means that our uniaxial medium has a hyperbolic dispersion relation $k_{\perp}^{2} / \varepsilon_{\|}+k_{\|}^{2} / \varepsilon_{\perp}=k_{0}^{2}$ [31]; it behaves as a normal dielectric along the one direction (vacuum, $\varepsilon_{\|}=1$ ) and as a metal along the other $\left(\varepsilon_{\perp}<0\right)$.

It is remarkable that when evaluating (3.2) at the specific angle (3.3), one understands that only one of the wavenumbers tends to infinity; the other remains finite. In particular:

$$
\begin{aligned}
& \frac{k_{z}^{(1)}}{k_{0}} \sim \begin{cases}\frac{\varepsilon_{\perp} \cos \phi \cot \phi-\sin \phi}{2 \sqrt{-\varepsilon_{\perp}}}, & \phi<0, \\
\frac{|\sin \phi|}{\theta_{0}-\theta}, & \phi>0,\end{cases} \\
& \frac{k_{z}^{(2)}}{k_{0}} \sim \begin{cases}\frac{|\sin \phi|}{\theta-\theta_{0}}, & \phi<0, \\
\frac{\varepsilon_{\perp} \cos \phi \cot \phi-\sin \phi}{2 \sqrt{-\varepsilon_{\perp}}}, & \phi>0\end{cases}
\end{aligned}
$$

for $\theta \rightarrow \theta_{0}$. It is clear that in proportion to which side we illuminate the structure, the same wave can have infinite or finite propagation constant. Furthermore, when one works close to $\theta=\theta_{0}$, only one of the two waves exhibits $k_{z} \rightarrow \infty$; the other one has a limiting propagation constant $k_{z}=\frac{\varepsilon_{\perp} \cos \phi \cot \phi-\sin \phi}{2 \sqrt{-\varepsilon_{\perp}}}$; when the side of excitation changes, the two waves switch position. Therefore, we have just introduced a regime $\left(\theta=\theta_{0}\right)$ at which the two modes supported by the same medium are infinitely different each other. Hereinafter, all the wave characteristics and the considered examples will mainly get examined in the parametric vicinity of that extreme asymmetry state: $\theta \rightarrow \theta_{0}$.

The waves can have nonzero imaginary part of their propagation constant only if the quantity under the square root of (3.2) is negative. It is straightforward to obtain:

$$
\operatorname{Im}\left[k_{z}\right] \neq 0 \Rightarrow \begin{cases}\theta>\arccos \left(\frac{\cos \phi}{\sqrt{1-\varepsilon_{\perp}}}\right), & \varepsilon_{\perp}<0 \\ \theta<\arccos \left(\frac{\cos \phi}{\sqrt{1-\varepsilon_{\perp}}}\right), & 0<\varepsilon_{\perp}<1\end{cases}
$$

which means that even with a lossless uniaxial medium, one can excite waves into it which have changing magnitude along $z$ by tilting properly the anisotropy axis (and selecting $\varepsilon_{\perp}<1$ ). Note that in case $\operatorname{Im}\left[k_{z}\right] \neq 0$, the imaginary parts of the two waves $k_{z}^{(1)}, k_{z}^{(2)}$ are opposite each other. It is also noteworthy that $\operatorname{Im}\left[k_{z}\right]$ is always zero in the vicinity of $\theta=\theta_{0}$ because $\theta_{0}<\arccos \left(\frac{\cos \phi}{\sqrt{1-\varepsilon_{\perp}}}\right)$. The same conclusion can be reached if we consider the ranges of $\varepsilon_{\perp}$ leading to nonzero $\operatorname{Im}\left[k_{z}\right]$. More specifically, if $\cos (2 \theta)>\cos (2 \phi)$ we have complex $k_{z}$ for $0<\varepsilon_{\perp}<\frac{\cos (2 \theta)-\cos (2 \phi)}{2 \cos ^{2} \theta}$; on the other hand, if $\cos (2 \theta)<$ $\cos (2 \phi)$, one obtains $\operatorname{Im}\left[k_{z}\right] \neq 0$ when $\frac{\cos (2 \theta)-\cos (2 \phi)}{2 \cos ^{2} \theta}<$ $\varepsilon_{\perp}<0$. However, the permittivity $\varepsilon_{\perp}=\frac{\cos (2 \theta)-1}{\cos (2 \theta)+1}<0$, leading 


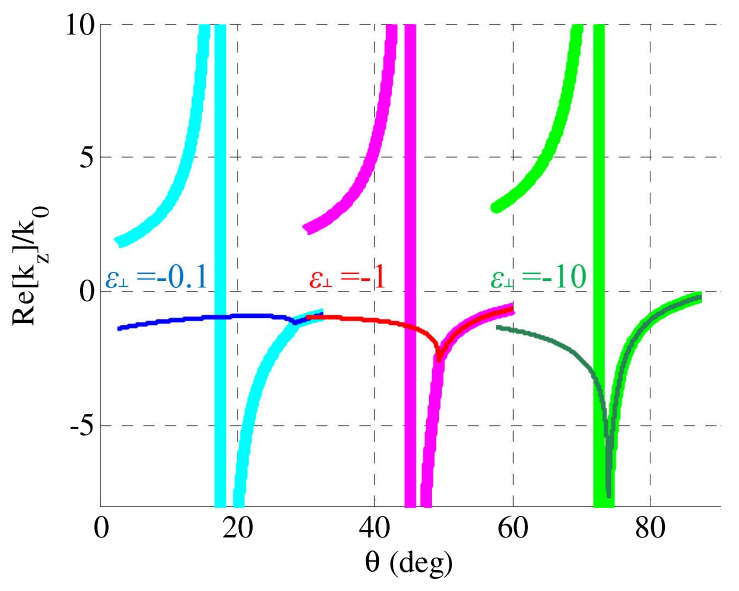

(a)

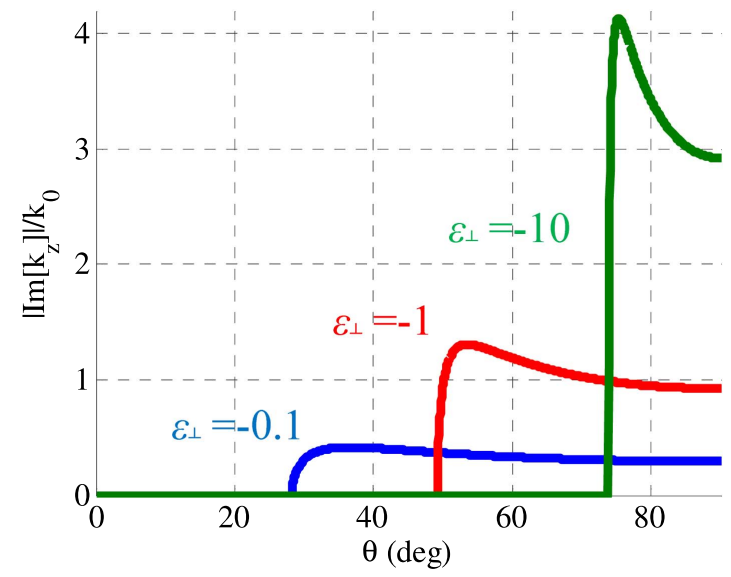

(b)

Figure 6 (a) The real part of the normalised wavenumbers $\operatorname{Re}\left[k_{z}\right] / k_{0}$ of the two waves (light and dark colours) and (b) the common absolute value of their imaginary part $\left|\operatorname{Im}\left[k_{z}\right]\right| / k_{0}$, as functions of the tilt angle $\theta$ for various negative transversal permittivities $\varepsilon_{\perp}=-0.1,-1,-10$. Plot parameter: $\phi=22.5^{\circ}$.

to the extreme-asymmetry regime, does not fall within the aforementioned ranges; it is always negative and smaller than $\frac{\cos (2 \theta)-\cos (2 \phi)}{2 \cos ^{2} \theta}$. If we had a half-space filled with the uniaxial medium instead of the finite slab of figure 5 , the selection of the correct solution would be made on the basis of the sign of the imaginary parts. Furthermore, in the case of $\operatorname{Im}\left[k_{z}\right]=0$, one can consider negligible but nonzero losses for $\varepsilon_{\perp}$. Finally, in all the following numerical results, graphs and contour plots of this section, the derived formulas are evaluated in MATLAB computing environment with use of standard built-in functions and in-house developed source code.

In figure 6(a), we demonstrate the asymmetry between the two modes described above. The real parts of the two wavenumbers $\operatorname{Re}\left[k_{z}^{(1,2)}\right] / k_{0}$ from (3.2) are shown as function of tilt angle $\theta$ for three different transversal permittivities $\varepsilon_{\perp}$. The first one $\left(\operatorname{Re}\left[k_{z}^{(1)}\right] / k_{0}\right)$ is represented with light thick line and the second one $\left(\operatorname{Re}\left[k_{z}^{(2)}\right] / k_{0}\right)$ with dark thin line. Since we have selected $\phi=22.5^{\circ}>0$, it is always the first wave that can possess infinite wavenumber as indicated by (3.4); this happens at different $\theta=\theta_{0}$ for various $\varepsilon_{\perp}$ according to (3.3). We notice that the two wavenumbers have a highly dissimilar $\theta$-variation especially in the vicinity of $\theta=\theta_{0}$ as totally anticipated. However, when $\theta$ goes above a threshold, the real parts of the two wavenumbers are identical which means that nonzero imaginary parts are appeared; such an effect is indicated by (3.5). Indeed in figure 6(b), where the (common) absolute value of imaginary part of the two $k_{z}$ is depicted as function of $\theta$, one observes that it has non-trivial variation for the same interval of $\theta$ that the couples of curves in figure 5 coincide each other. This feature is attributed to the lossless $\varepsilon_{\perp} \in \mathbb{R}$ and the presence of square root in (3.2) for $\varepsilon_{\|}=1$.

\subsection{Wave impedances and perfect matching regime}

It worths mentioning that the impedances of the two waves defined by the supported wavenumbers (3.2) are either equal or opposite each other. If one evaluates the ratio between the transversal (with respect to the $z$ propagation axis) electric component ( $x$ direction) over the transversal (and unique) magnetic component ( $y$ direction), one obtains:

$$
Z= \pm \eta_{0} \frac{\sqrt{\varepsilon_{\perp}\left[\varepsilon_{\perp}+\left(\varepsilon_{\perp}-1\right) \cos (2 \theta)+\cos (2 \phi)\right]}}{\varepsilon_{\perp} \sqrt{2}}
$$

where $\eta_{0}=\sqrt{\mu_{0} / \varepsilon_{0}}$ is the free-space wave impedance. Note that $Z$ is either purely real or purely imaginary for a lossless medium $\left(\varepsilon_{\perp} \in \mathbb{R}\right)$. If $\varepsilon_{\perp}=1$, we obtain $Z=Z_{0}= \pm \eta_{0} \cos \phi$, namely the well-known TM impedances of waves travelling into vacuum either along one $(+z)$ or the opposite $(-z)$ direction. This is not the case when $\varepsilon_{\perp} \neq 1$; the two waves (with superscripts 1 or 2 ) do not necessarily retain the same sign for their impedance in (3.6) regardless of the variations in angles $(\theta, \phi)$ and permittivity $\varepsilon_{\perp}$. As indicated by (3.4), the two waves usually change positions in proportion to the signs of the real and imaginary part of their $k_{z}$, which in turn are determined by the sign of angle $\phi$ and the ranges of other parameters.

If one evaluates the wave impedances (3.6) at the angle $\theta=\theta_{0}$, where the extremal asymmetry is occurred, one obtains the following limiting expression: $Z=\mp \eta_{0}|\sin \phi| / \sqrt{-\varepsilon_{\perp}}$ for $\theta=\theta_{0}$ (where $\varepsilon_{\perp}<0$ ). By comparing it with the formula for the TM wave impedance $Z_{0}$, we can directly find the condition for perfect matching between our medium and free-space:

$\lim _{\theta \rightarrow \theta_{0}} Z=Z_{0} \Rightarrow \eta_{0} \frac{|\sin \phi|}{\sqrt{-\varepsilon_{\perp}}}=\eta_{0} \cos \phi \Rightarrow \varepsilon_{\perp}=-\tan ^{2} \phi$.

This set of parameters combines two unique features for the medium under investigation: (i) extreme asymmetry (one $k_{z}$ finite, the other infinite) of the supported modes $\left(\theta=\theta_{0}\right)$ and (ii) perfect matching with free-space $\left(Z=Z_{0}\right)$. Note that such a case corresponds to $\phi= \pm \theta_{0}$, and to $\varepsilon_{z z}=0$, while the off diagonal permittivities $\varepsilon_{x z}$ are non-zero. 


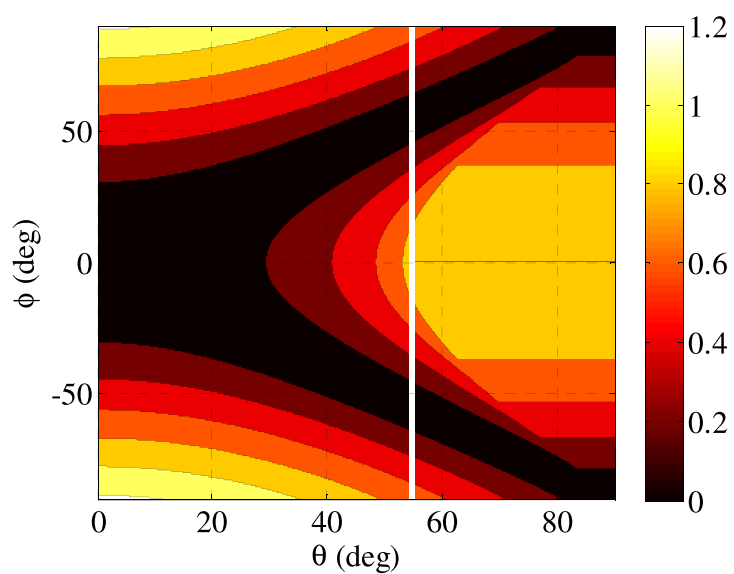

(a)

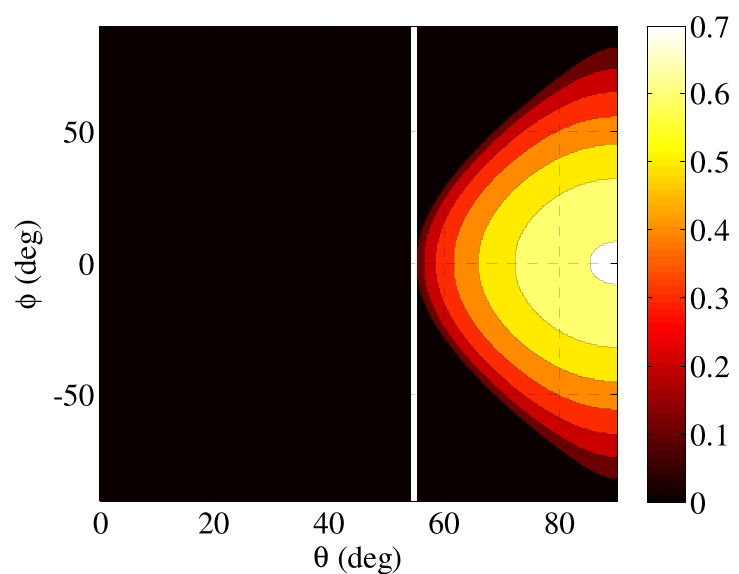

(b)

Figure 7. (a) The normalised difference $\left|Z / \eta_{0}-\cos \phi\right|$ between the real part of the common wave impedance $Z$ and the TM wave impedance $Z_{0}=\eta_{0} \cos \phi$. (b) The imaginary part of the normalised common wave impedance $Z / \eta_{0}$ of the two waves, represented as function of the tilt angle $\theta$ and the incidence angle $\phi$. The white lines correspond to the tilt angle $\theta=\theta_{0}$ leading to the extreme asymmetry regime. Plot parameter: $\varepsilon_{\perp}=-2$.

In figure 7(a), we represent the difference between the wave impedance $Z$ and the TM impedance into free space $Z_{0}=\eta_{0} \cos \phi$ showing how far we are from the matching regime. The variation of the quantity $\left|Z / \eta_{0}-\cos \phi\right|$ is shown with respect to the tilt and incidence angles $(\theta, \phi)$. With the white line we mark the angle $\theta_{0}$ for extremal mode asymmetry and we see that perfect matching at $\theta=\theta_{0}$ is only achieved when the direction of the incident field becomes parallel $\left(\phi=\theta_{0}\right)$ or symmetric $\left(\phi=-\theta_{0}\right)$ to the optical axis of the uniaxial medium. As far as $\operatorname{Im}\left[\varepsilon_{\perp}\right]$ is concerned (figure 7(b)), it has small values for most combinations $(\theta, \phi)$ including the $\theta=\theta_{0}$ case (white line) and increases abruptly for a quasi-normal illumination and very large tilt angles $\theta$. That means that for $\phi= \pm \theta_{0}$ the matching $Z=Z_{0}$ is not hindered by larger $\operatorname{Im}[Z]$.

In the following, let us focus on a characteristic case: $\tan \phi= \pm 1 \Rightarrow \phi= \pm 45^{\circ}$ which leads to $\varepsilon_{\perp}=-1$; similar results are reported for $\varepsilon_{\|} \neq 1$ and $\varepsilon_{\perp} \neq-1$ but we confined ourselves mainly to this combination of permittivities for brevity and better understanding of the effect. In that case, we obtain: $\varepsilon_{x x}=\varepsilon_{z z}=0$ and $\varepsilon_{x z}=\varepsilon_{z x}= \pm 1$. Such a regime $\left(\theta=\phi= \pm 45^{\circ}\right)$ will be extensively analysed but let us first examine in the next subsection how the desired hyperbolic properties $\left(\varepsilon_{\|}>0\right.$ and $\left.\varepsilon_{\perp}<0\right)$ are emulated by actual materials.

\subsection{Graphene multilayers as hyperbolic medium}

One of the most common way to build a medium that is characterised by effective hyperbolic properties is to use thin layers of plasmonic materials alternated by slabs of ordinary host (vacuum in the simplest case) [41]. Graphene can be isolated and constitute a very flexible and durable $2 \mathrm{D}$ medium [44] which if it gets suitably doped with electric charge carriers, exhibits remarkable plasmonic properties by supporting both high- and low-energy plasmons [60]. For this reason, we are going to achieve plasmonic $\varepsilon_{\perp}$ by stacking numerous graphene multilayers to form a lattice with a small spatial period.

The crucial quantity characterising the operation of graphene is the complex surface conductivity $\sigma$ (measured in $1 / \Omega$ ) which expresses how easily the carriers of electric charge (electrons) are moving on the surface of the medium. It is comprised of two terms: one corresponding to the transition of electrons within the same energy level (intraband transitions, low-energy plasmons, $\sigma_{\text {intra }}$ ) and another describing the hop of charges to neighbouring energy levels (interband transitions, high-energy plasmons, $\left.\sigma_{\text {inter }}\right)$. The related formulas are extracted with use of Kubo model [46] for conductivity and their approximate versions are given below:

$$
\begin{gathered}
\sigma_{\text {intra }} \cong \mathrm{i} \frac{e^{2} k_{B} T}{\pi \hbar^{2}\left(\omega+\mathrm{i} \tau^{-1}\right)}\left(\frac{\mu_{c}}{k_{B} T}+2 \ln \left(\mathrm{e}^{-\mu_{c} / k_{B} T}+1\right)\right), \\
\sigma_{\text {inter }} \cong \mathrm{i} \frac{e^{2}}{4 \pi \hbar} \ln \left(\frac{2 \mu_{c}-\left(\omega+\mathrm{i} \tau^{-1}\right)}{2 \mu_{c}+\left(\omega+\mathrm{i} \tau^{-1}\right)}\right),
\end{gathered}
$$

where $\mu_{c}$ is the chemical potential expressing the energy transferred to the graphene flake during the doping with electrons (of charge $e$ ) and $\tau$ is the relaxation time of charges. The symbols $k_{B}$ and $\hbar$ are used for the Boltzmann constant and the reduced Planck constant respectively, while $T$ is the local temperature. The total surface conductivity of graphene is the sum of the aforementioned two terms: $\sigma=\sigma_{\text {intra }}+\sigma_{\text {inter }}$.

We are planning to use graphene to mimic hyperbolic properties in operational wavelengths $\lambda=2 \pi c / \omega \quad(c=$ $1 / \sqrt{\varepsilon_{0} \mu_{0}}$ is the speed of light into vacuum) covering the lower part of visible spectrum and higher part of mid-infrared: $0.5 \mu \mathrm{m}<\lambda<3 \mu \mathrm{m}$. Feasible values for the chemical potential are between 0.2 and $1.2 \mathrm{eV}$, while the relaxation time can vary substantially within the limits: $0.01 \mathrm{ps}<\tau<1 \mathrm{ps}$. The temperature is kept fixed throughout the considered examples: $T=300 \mathrm{~K}$.

Back into our configuration of figure 5 , we assume that the slab of thickness $h$ is comprised of a dense lattice of parallel graphene flakes which are tilted with respect to $z$ axis 


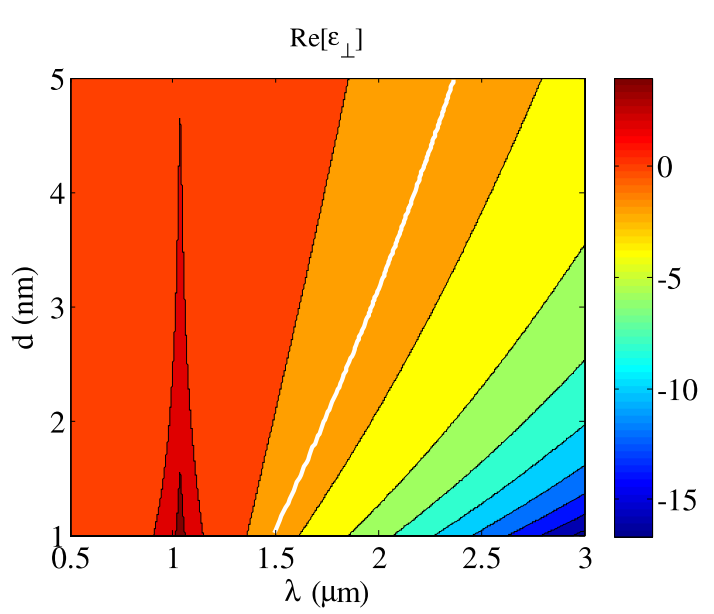

(a)

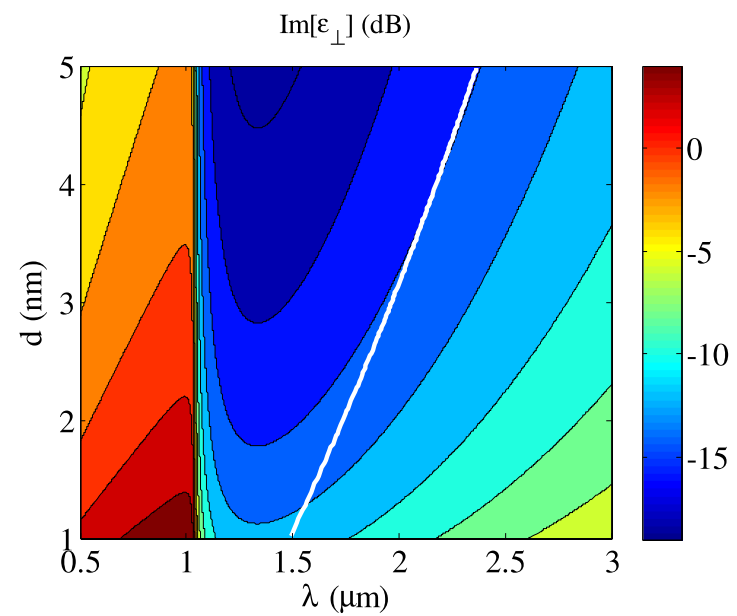

(b)

Figure 8. The: (a) real part $\operatorname{Re}\left[\varepsilon_{\perp}\right]$ and (b) imaginary part $10 \log \left(\operatorname{Im}\left[\varepsilon_{\perp}\right]\right)$ in $\mathrm{dB}$ of the effective transversal permittivity $\varepsilon_{\perp}$ emulated by a freestanding cluster of parallel graphene flakes, represented on the map of the operational wavelength $\lambda$ and the lattice period $d$. The white lines correspond to $\operatorname{Re}\left[\varepsilon_{\perp}\right]=-1$. Plot parameters: $\mu_{c}=0.6 \mathrm{eV}, \tau=0.1 \mathrm{ps}$.

by angle $\theta$. The distance between two neighbouring graphene sheets is $d$ and they are infinite along $y$ axis and finite on $z x$ plane. The period $d$ of the structure is much smaller than the oscillating wavelength $\lambda$ so that the whole structure behaves effectively as one medium. The background material into which we locate the graphene flakes, is vacuum $\left(\varepsilon_{\|}=1\right)$ as indicated above, for brevity. Under the assumption that $d \ll \lambda$, one can treat the cluster of graphene macroscopically as a homogeneous medium. Obviously $\varepsilon_{\|}=1$, equal to the host material's (vacuum), because the $2 \mathrm{D}$ media cannot interact with electric fields normal to their surface. As far as the effective transversal permittivity of the free-standing graphene lattice is concerned, it is given by $[59,60]$ :

$$
\varepsilon_{\perp} \cong \varepsilon_{\|}+\mathrm{i} \frac{\sigma \eta_{0}}{k_{0} d}=1+\mathrm{i} \frac{\sigma \eta_{0}}{k_{0} d} .
$$

The expression (3.10) is derived by evaluating ordinary mixing formulas when one of the two materials gets infinitely concentrated on a surface (2D medium graphene).

It would be interesting to examine the possibility of achieving through (3.10), a macroscopic perpendicular permittivity $\varepsilon_{\perp} \cong-1$ which leads to perfect matching with free space as dictated by (3.7) for $\phi= \pm 45^{\circ}$. One could point out that when $\theta=45^{\circ}$, the matching (zero reflection) for $\phi=45^{\circ}$ becomes trivial because the incident field does not 'see' the two-dimensional graphene and thus the only case one should examine only the case for $\phi=-45^{\circ}$. This objection is only partially correct: we are interested not only for zero reflection but for perfect absorption with slightly lossy plasmonic media which is not trivial at all; therefore we investigate both the incident angles $\phi= \pm 45^{\circ}$ whose behaviour is very similar in terms of absorption. We are considering a lattice of graphene flakes with period $1 \mathrm{~nm}<d<5 \mathrm{~nm}$ and typical value $d=3 \mathrm{~nm}$; all the other parameters are varying within the ranges defined above. In figure 8 , we show the variation of the real and the imaginary part of $\varepsilon_{\perp}$ according to (3.10) with respect to the time oscillation wavelength $\lambda$ and the distance $d$ between two successive graphene flakes. In figure 8(a), one clearly notices that very negative values of effective $\varepsilon_{\perp}$ are obtained for larger wavelengths. Therefore, the regime $\operatorname{Re}\left[\varepsilon_{\perp}\right]=-1$ can be achieved for all the considered lattice periods $d$ if one chooses properly the operating wavelength, as indicated by the white line giving the suitable combinations of $(\lambda, d)$. By observing figure $8(\mathrm{a})$, one can clearly spot the large change in $\operatorname{Re}\left[\varepsilon_{\perp}\right]$ from lower to higher $\lambda$ which is owed to the vanishing interband transitions as frequency decreases. A similar and more obvious switch is appeared for the same reason in figure $8(\mathrm{~b})$, where the imaginary part of $\varepsilon_{\perp}$ is represented in $\mathrm{dB}: 10 \log \left|\operatorname{Im}\left[\varepsilon_{\perp}\right]\right|$. It should be also stressed that the losses along the white line of $\operatorname{Re}\left[\varepsilon_{\perp}\right]=-1$ are very low which means that the perfect matching purpose of (3.7) can be served well by the tilted stack of graphene sheets into vacuum.

In figure 9, we fix both the operational wavelength $\lambda$ and the distance $d$ and we represent $\varepsilon_{\perp}$ as function of relaxation time $\tau$ and chemical potential $\mu_{c}$; the combinations of $\left(\tau, \mu_{c}\right)$ giving $\operatorname{Re}\left[\varepsilon_{\perp}\right]=-1$ are again indicated by the white solid line. One clearly remarks that in order to emulate a negative $\operatorname{Re}\left[\varepsilon_{\perp}\right]$ (figure 8), highly doped (increased $\mu_{c}$ ) graphene is required which is sensible because the strong plasmons [60] excited across the flakes are essential. The dependence from $\tau$ is weak for $\operatorname{Re}\left[\varepsilon_{\perp}\right]<0$ but scattering time plays a role for low doping. In figure 9(b), we show the variation of $\operatorname{Im}\left[\varepsilon_{\perp}\right]$ on the same map and again we record negligible losses for the interesting state indicated by (3.7).

\subsection{Perfectly absorbing configuration}

The regime described by the conditions (3.7) which combines two interesting features (extremal mode asymmetry, perfect matching with free space) can be of high practical importance and is the objective of this subsection. When the incident illumination meets the front surface of the slab at $z=h / 2$ if (3.7) is valid, no reflections will occur due to perfect 


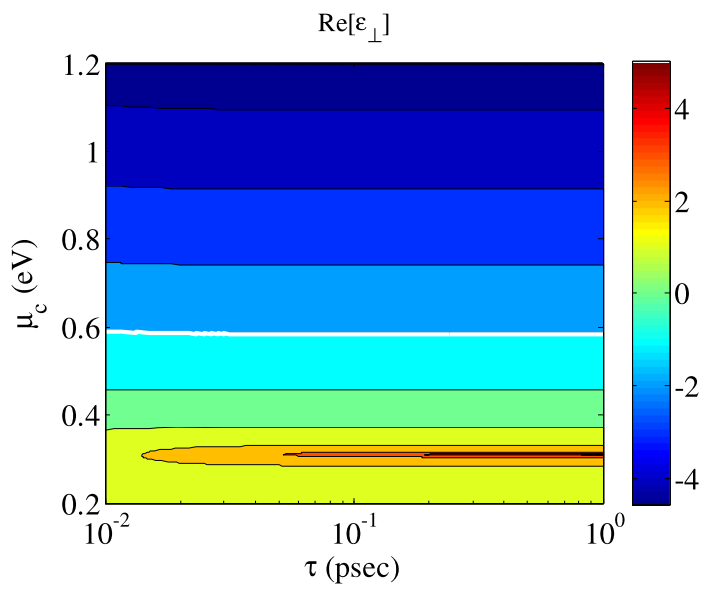

(a)

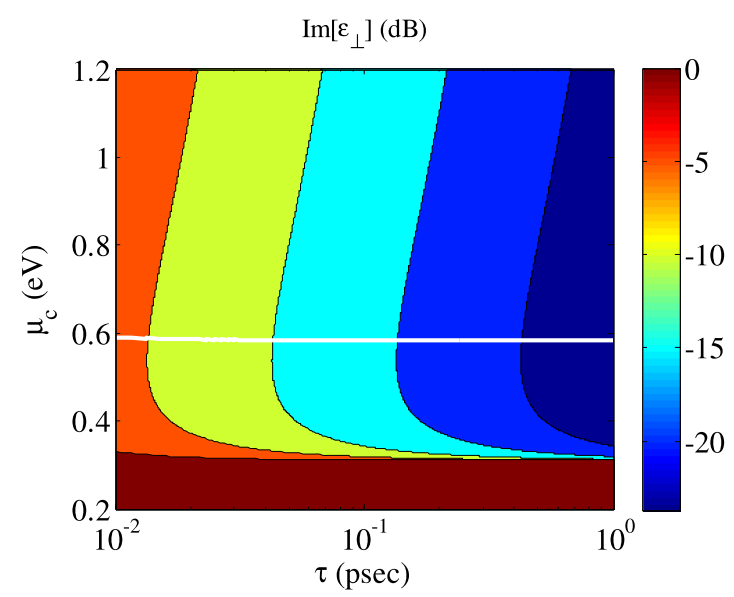

(b)

Figure 9. The: (a) real part $\operatorname{Re}\left[\varepsilon_{\perp}\right]$ and (b) imaginary part $10 \log \left(\operatorname{Im}\left[\varepsilon_{\perp}\right]\right)$ in $\mathrm{dB}$ of the effective transversal permittivity $\varepsilon_{\perp}$ emulated by a freestanding cluster of parallel graphene flakes, represented as functions of electrons transport scattering time $\tau$ and the chemical potential of doped graphene $\mu_{c}$. The white lines correspond to $\operatorname{Re}\left[\varepsilon_{\perp}\right]=-1$. Plot parameters: $\lambda=2 \mu \mathrm{m}, d=3 \mathrm{~nm}$.

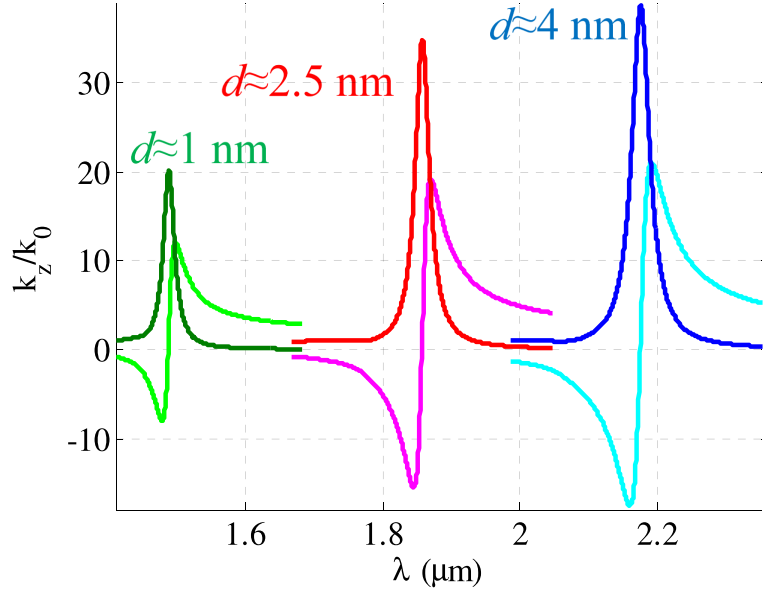

Figure 10. The change of the real (light colours) and the imaginary (dark colours) parts of the wavenumber $k_{z} / k_{0}$ with respect to operational wavelength $\lambda$ for three different lattice periods $d \cong 1,2.5,4 \mathrm{~nm}$. Plot parameters: $\phi=22.5^{\circ}, \theta=45^{\circ}$, $\mu_{c}=0.6 \mathrm{eV}, \tau=0.1 \mathrm{ps}$.

matching; therefore, all the power of the input will enter the slab. Within the slab, the input wave will excite the mode with huge $\operatorname{Re}\left[k_{z}\right]$ and due to the inevitable losses of the device (in the case of graphene, they are hidden in the real part of conductivity $\sigma$ from (3.8) and (3.9)), will lose the vast portion of its carrying power before reaching the rear interface $z=-h / 2$. That is because even the slightest $\operatorname{Im}\left[\varepsilon_{\perp}\right]=\operatorname{Re}[\sigma] \eta_{0} / k_{0} d$ (from (3.10)) will lead to a sizable $\operatorname{Im}\left[k_{z}\right]$ due to the huge $\operatorname{Re}\left[k_{z}\right]$. Accordingly, the quantity $\operatorname{Im}\left[k_{z}\right] h$ would be fairly large even for tiny thicknesses $h$ to fully absorb the travelling wave. This characteristic is not apparent in figure 6 because a lossless host medium $\left(\operatorname{Im}\left[\varepsilon_{\perp}\right]=0\right)$ is considered.

For this reason, we show in figure 10 the variation of $k_{z} / k_{0}$ for the realistic (and thus slightly lossy) case of graphene multilayers. In particular, we consider three different lattice thicknesses $d \cong 1,2.5,4 \mathrm{~nm}$ and we examine the change of the real and the imaginary part of that wavenumber which takes theoretically unbounded values (according to (3.4) referring to the lossless scenario), in the neighbourhood of the operational frequency at which $\operatorname{Re}\left[\varepsilon_{\perp}\right] \cong-1$ according to figure 8 . The tilt of the optical axis is of course $\theta=45^{\circ}$ as mentioned earlier. It is clear that $k_{z}$, in all cases, experiences Lorentz resonance around wavelength $\lambda$ at which vicinity the equality $\operatorname{Re}\left[\varepsilon_{\perp}\right]=-1$ occurs. Even with the very small imaginary part of the perpendicular permittivity $\operatorname{Im}\left[\varepsilon_{\perp}\right]$ as appears along the white line of figure $8(b)$, we obtain extremely high imaginary part for the supported propagation constants $\operatorname{Im}\left[k_{z}\right]$ (tens of times of the free-space wavenumber $k_{0}$ ) which, combined with perfect matching, guarantees extremely high absorptions. Finally, the denser is the lattice, the higher is the damping factor $\exp \left(-\operatorname{Im}\left[k_{z}\right] h\right)$ of the hyperbolic medium and thus the absorbing effect is expected to be stronger.

Let us verify the conjectures for the significant absorbing potential of a thin layer populated by tilted graphene multilayers under the conditions (3.7). The slab of figure 5 is illuminated by an obliquely incident TM wave with magnetic field: $\mathbf{H}_{\text {inc }}=$ $\hat{\mathbf{y}} H_{0} \exp \left[-\mathrm{i} k_{0}(x \sin \phi+z \cos \phi)\right]$ and the corresponding magnetic fields for the reflected and transmitted waves would be given by: $\mathbf{H}_{\mathrm{ref}}=\hat{\mathbf{y}} R H_{0} \exp \left[-\mathrm{i} k_{0}(x \sin \phi-z \cos \phi)\right]$ and $\mathbf{H}_{\text {trans }}=\hat{\mathbf{y}} T H_{0} \exp \left[-\mathrm{i} k_{0}(x \sin \phi+z \cos \phi)\right]$. The complex reflection and transmission coefficients are derived after imposing the essential boundary conditions at $z= \pm h / 2$ but are not shown for brevity since their formulas are quite lengthy. Obviously, the quantity $|R|^{2}$ gives the portion of the incident power that is reflected, the quantity $|T|^{2}$ corresponds to the portion that is transmitted and $A=1-|R|^{2}-|T|^{2}$ is the absorbed power by the thin graphene-based hyperbolic slab.

In figure 11 , we consider the three cases of $d \cong 1,2.5$, $4 \mathrm{~nm}$ examined in figure 10 for incident illumination $\phi=45^{\circ}$ as dictated by (3.7). In the same graph we represent the absorption $A$ and the transmitted power $|T|^{2}$ as function of the oscillating wavelength $\lambda$. First of all we have perfect absorption of the incident field not only at the frequency that $\operatorname{Re}\left[\varepsilon_{\perp}\right]=-1$ is achieved (from the map of figure 8(a)) but for a range of 


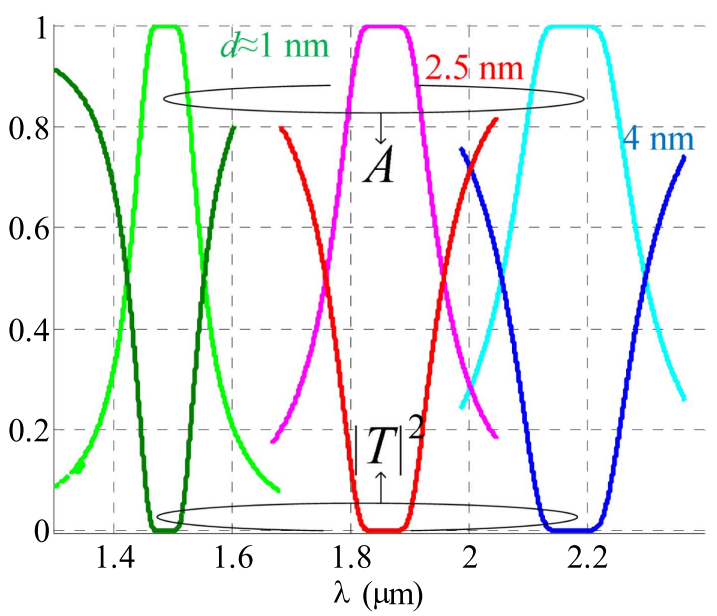

(a)

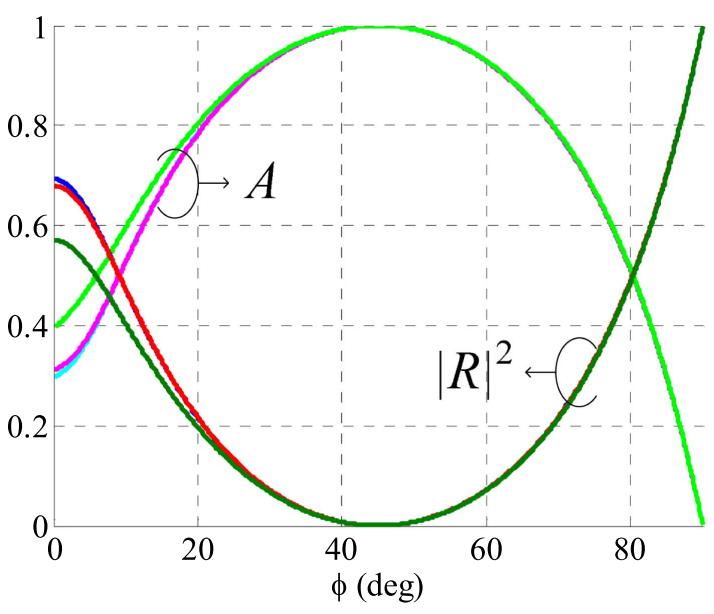

(b)

Figure 11. (a) The absorbing $A$ (light colours) and the transmitted $|T|^{2}$ (dark colours) power from the slab as functions of the operating wavelength $\lambda$ for three different lattice periods $d \cong 1,2.5,4 \mathrm{~nm}$ and $\phi=45^{\circ}$. (a) The absorbing $A$ (light colours) and the reflected $|R|^{2}$ (dark colours) power from the slab as functions of the incidence angle $\phi$ for the same thicknesses $d$ working at their optimal wavelengths $\lambda$. Common plot parameters: $\theta=45^{\circ}, \mu_{c}=0.6 \mathrm{eV}, \tau=0.1 \mathrm{ps}, h=100 \mathrm{~nm}$.

wavelengths around it. Therefore, we report wideband perfect absorption and the width of the fully absorbing zone becomes smaller as $d$ decreases. The transmission explodes far from the optimal frequency and is complementary to $A$ because the reflection coefficient is trivially zero $\left(\phi=\theta=45^{\circ}\right)$. In figure 11(b), we regard the same configurations as in figure 11(a) but now they are working at different frequencies: each one at its optimal wavelength extracted from figure 8(a); the behaviour is similar regardless of the choice of $d$. In this graph, reflection is not zero because the incidence angle $\phi$ changes; thus, we show the variation of $A$ and $|R|^{2}$ which are almost complimentary each other (negligible transmission). It is noteworthy that the absorbing performance of our device exhibits substantial endurance with respect to $\phi$, which additionally leads to wideangle absorption. As far as the reflection coefficient is concerned, it becomes larger when moving towards normal incidence and of course we take $|R|^{2}=1$ for the grazing angle $\left(\phi=90^{\circ}\right)$ illumination.

In figure 12, we pick one of the cases examined in figure 11 (the one with $d=4 \mathrm{~nm}$ ) and show in contour plot the variation of the absorption $A$ on the map of the wavelength spectrum $\lambda$ and the incidence spectrum $\phi$. Obviously a 'plateau' of perfect absorption is created around the optimal frequency and $\phi=45^{\circ}$, which is quite extensive leading to both wideband and wide-angle behaviour (as indicated in figure 11).

In figure 13, we examine the effect of the graphene characteristics $\left(\tau, \mu_{c}\right)$ and the thickness of the slab $h$ on the absorbing performance $A$ of our system for a wavelengths range. In figure 13(a), we notice that larger transport scattering time of the electrons leads to more narrow absorbing bands around the same optimal wavelength $\lambda$. This could be anticipated from figure 9(b), where it is shown that higher $\tau$ leads to less lossy $\varepsilon_{\perp}$ and thus to sharper resonances. On the contrary, the maximal $A$ when $\mu_{c}$ is varying is exhibited at different frequencies (figure 13(b)). In particular, the

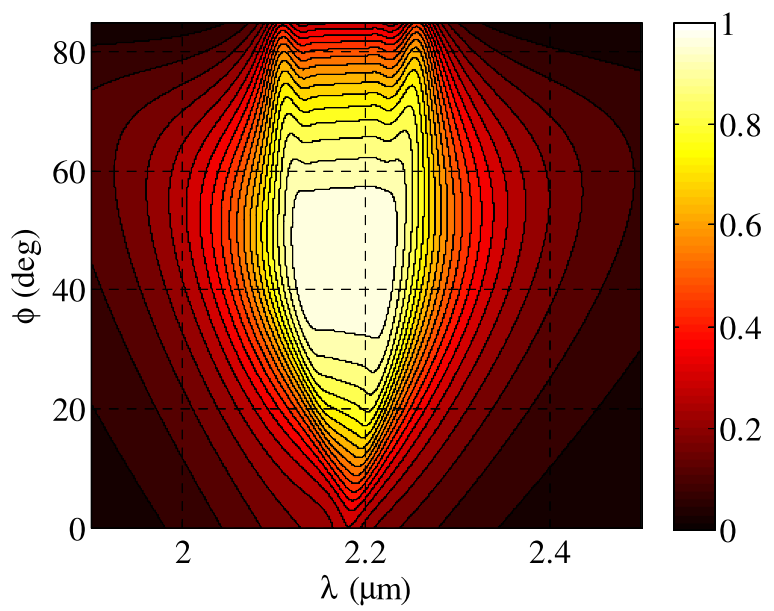

Figure 12. The absorbed power $A$ by the slab on the spectra plane of the wavelength $\lambda$ and incidence angle $\phi$. Plot parameters: $\theta=45^{\circ}$, $\mu_{c}=0.6 \mathrm{eV}, \tau=0.1 \mathrm{ps}, h=100 \mathrm{~nm}, d=4 \mathrm{~nm}$.

frequency responses of $A=A(\lambda)$ are equally sharp regardless of $\mu_{c}$, while the optimal wavelength becomes smaller as the chemical potential of the doped graphene gets larger. In figure 13(c), we remark that a thicker device (larger $h$ ) gives the absorption a wideband character since increases the frequency range of the perfect absorption. It is noteworthy that the thickness resonance is absent due to the difference in propagation constants for upward and downward waves within the slab.

\subsection{Waveguiding configuration}

In this subsection, we examine the waves that can be guided into an asymmetric hyperbolic slab similar to that of figure 5 with the difference that is PEC backed from the bottom side $(z=-h / 2)$. Again the uniaxial medium with permittivities $\left(\varepsilon_{\|}, \varepsilon_{\perp}\right)$ is emulated with use of tilted graphene flakes 
(a)

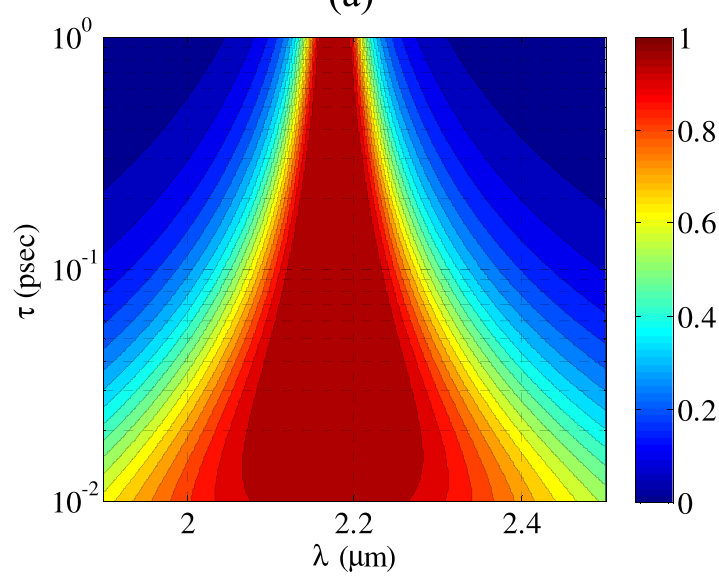

(b)

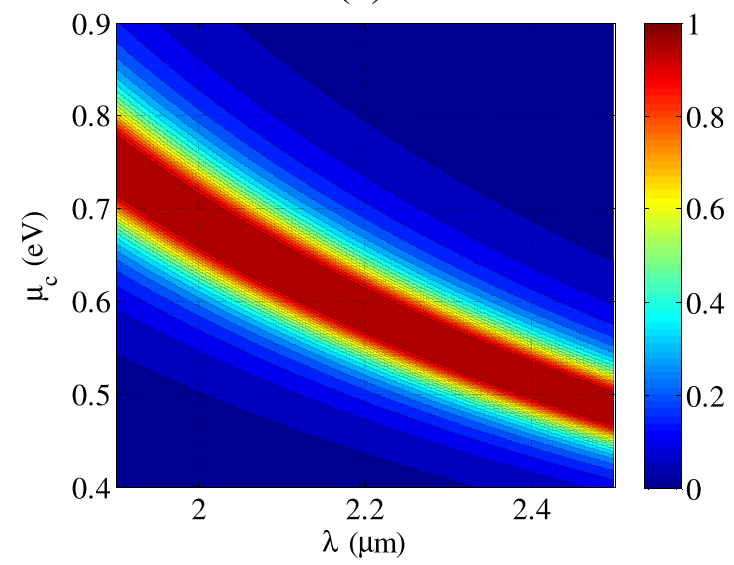

(c)

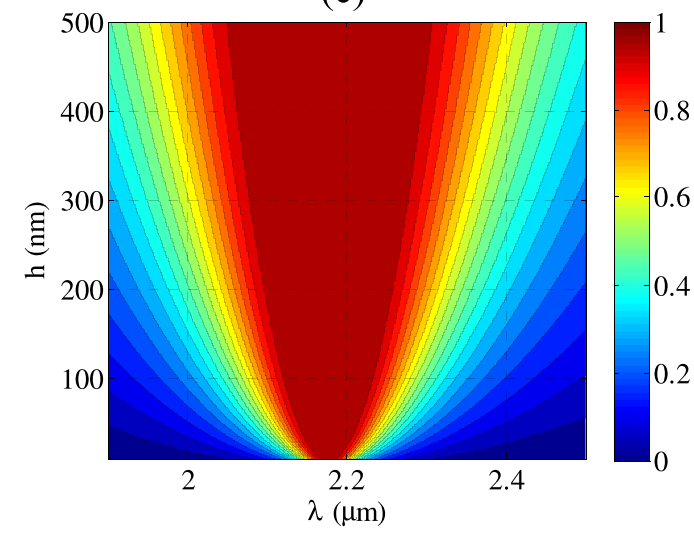

Figure 13. The absorbed power $A$ in contour plot as function of the operating wavelength $\lambda$ and: (a) the scattering time $\tau(h=100 \mathrm{~nm}$, $\left.\mu_{c}=0.6 \mathrm{eV}\right)$, (b) the chemical potential of the doped graphene $\mu_{c}(h=100 \mathrm{~nm}, \tau=0.1 \mathrm{ps})$ and (c) layer thickness $h(\tau=0.1 \mathrm{ps}$, $\left.\mu_{c}=0.6 \mathrm{eV}\right)$. Common plot parameters: $\theta=45^{\circ}, d=4 \mathrm{~nm}$.

characterised by the surface conductivity $\sigma$ as evaluated from (3.8) and (3.9). Obviously, the perpendicular permittivity $\varepsilon_{\perp}$ is dependent on the operating frequency $\omega=2 \pi f$, the chemical potential $\mu_{c}$ and the scattering time $\tau$. For simplicity, we regard the two-dimensional problem with fields independent from $y$ variable and consider waves travelling along $x$ axis with propagation constant $k_{x}$. The magnetic field is again assumed of the TM form: $\mathbf{H}=\hat{\mathbf{y}} \exp \left(\mathrm{i} k_{x} x\right) S(z)$, where $S(z)$ is a suitable function determined by the boundary conditions, and the waveguiding condition takes the form:

$$
\begin{aligned}
& \tan \left(\sqrt{\left(k_{0}^{2} \varepsilon_{z z}-k_{x}^{2}\right)\left(\varepsilon_{x z}^{2}-\varepsilon_{x x} \varepsilon_{z z}\right)} h / \varepsilon_{z z}\right) \\
& \quad=\frac{\left(\varepsilon_{x z}^{2}-\varepsilon_{x x} \varepsilon_{z z}\right) \sqrt{k_{x}^{2}-k_{0}^{2}}}{\sqrt{\left(k_{0}^{2} \varepsilon_{z z}-k_{x}^{2}\right)\left(\varepsilon_{x z}^{2}-\varepsilon_{x x} \varepsilon_{z z}\right)}} .
\end{aligned}
$$

The transcendental equation (3.11) can be numerically solved with respect to complex $k_{x}$ for a range of different frequencies $f=\omega / 2 \pi=c / \lambda$; in this way, the dispersion diagrams are obtained. Suitable built-in FORTRAN libraries containing efficient algorithms for finding roots of complex functions like (3.11) on the complex plane $\left(\operatorname{Re}\left[k_{x}\right], \operatorname{Im}\left[k_{x}\right]\right)$ have been used. Such equations possess a countable number of (families of) solutions, only few of which are indicatively represented in the following dispersion graphs.

In figure 14 , we show the real parts $\operatorname{Re}\left[k_{x}\right]$ of complex wavenumbers $k_{x}$ which satisfy (3.11) for a continuous spectrum of linear frequencies $f$ and three different tilt angles $\theta=0^{\circ}, 15^{\circ}, 25^{\circ}$ (different colour: green, blue, red); two families of solutions are represented for each $\theta$ (different markers: circles and squares). The cut-off frequency $f_{0} \cong 40.3 \mathrm{THz}$ separates the considered frequency band in two parts: for $f<f_{0}$, propagating modes $\left(\operatorname{Im}\left[k_{x}\right] \cong 0\right)$ having either positive or negative inverse group velocity $\operatorname{Re}\left[k_{x}^{\prime}(\omega)\right]$ (derivative of $\operatorname{Re}\left[k_{x}\right]$ with respect to circular frequency $\omega=2 \pi f$ ) are guided. On the contrary, for $f>f_{0}$ the regarded medium loses its hyperbolic nature and therefore leaky modes (with $\operatorname{Im}\left[k_{x}\right] \neq 0$ ) are appeared, which are very similar each other regardless of angle $\theta$. It is noteworthy that for $\theta=0$, namely when graphene sheets are orthogonal to waveguide boundaries, the propagating waves are characterised by $\operatorname{Re}\left[k_{x}^{\prime}(\omega)\right]<0$ which means that they are all backwarddirected. As far as the other tilt angles are concerned, the supported waves are backward for frequencies below a limit (which is lower for larger $\theta$ ) and forward-propagating above it. In particular, these frequency limits are $f \cong 38.8 \mathrm{THz}$ for $\theta=15^{\circ}$ and $f \cong 38 \mathrm{THz}$ for $\theta=25^{\circ}$ which play also the role of accumulation frequency points. Indeed, in the vicinity of each of them, all the propagating modes (for fixed $\theta$ ) get almost vanished $\left(\operatorname{Re}\left[k_{x}\right] \rightarrow 0\right)$ passing through the almost same point. When it comes to the cutoff frequency $f_{0}$, it is not dependent on $\theta$ but it acts also as accumulation point where 


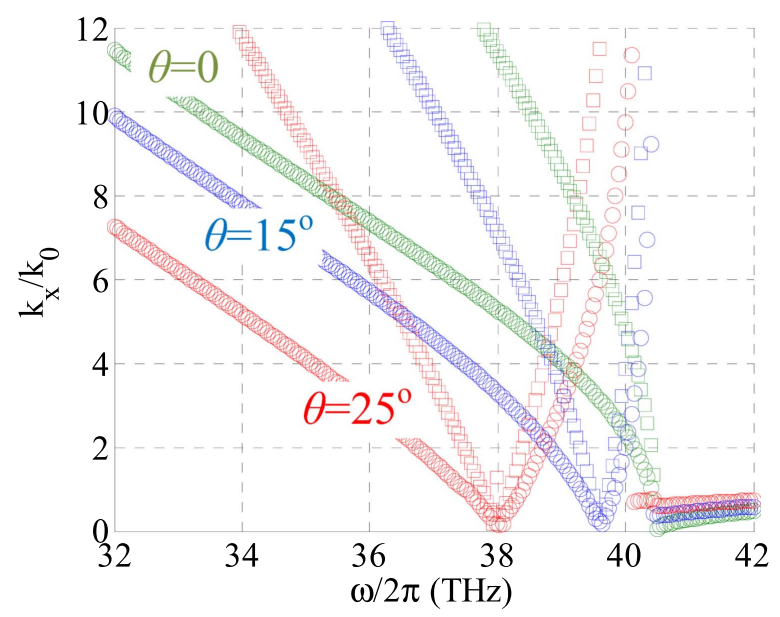

Figure 14. Representation of the solutions of (3.11) on the map of frequency $f$ and the real part of the normalised supported wavenumber along $x$ axis $\operatorname{Re}\left[k_{x}\right] / k_{0}$ for various tilt angles $\theta$. Plot parameters: $\mu_{c}=0.15 \mathrm{eV}, d=20 \mathrm{~nm}, h=300 \mathrm{~nm}, \tau=10 \mathrm{ps}$.

the waves change their behaviour (from propagating to leaky). Note that in the neighbourhoods of accumulation points, the wavevector component which is normal to slab boundaries $\left(k_{z}\right)$ becomes huge because close to these frequencies the Lorentz resonances of figure 10 are occurred.

From figure 14, it is obvious that the orientation of graphene sheets can engineer waveguides with required spectra of modes; characteristically, if graphene sheets are parallel to the waveguide boundaries, only plasmonic modes will get supported propagating along the flakes [60]. However, one can control the waves existing into such a component without changing its structural characteristics such as the doping of graphene. In figure 15, we show the dispersion curves of two families of modes obeying (3.11) for three different chemical potentials $\mu_{c}$. We notice again the presence of accumulation points which are being shifted towards larger frequencies for slight increase of doping energy $\mu_{c}$.

The guidance of backward and forward waves at the same frequency can offer the opportunity of matching their phases (acquiring the same $k_{x}$ ) as has been reported in [53-55]. In particular, we notice from figures 14 and 15 that backward modes are supported for smaller frequencies than the forward modes for a specific selection of $\theta$ and $\mu_{c}$. If one assumes nonlinearity, then the same types of modes appeared on $\left(f, k_{x}\right)$ plane around accumulation frequency $f=f_{0}$, will get reproduced at larger frequencies (higher-order harmonics). Therefore, the possibility of matching for a specific waveguide and at a certain frequency $f$ a forward-propagating wave with a backward-propagating mode of a higher harmonic becomes feasible. Such potential of phase matching in a slab of the asymmetric hyperbolic material is illustrated in figure 16, where the dispersion curves of three backward modes (green) and three forward modes (blue) are shown. The red curve represents the fictitious dispersion of one of the backward waves excited at a higher harmonic: in such a conditional scenario, the matching will be achieved at their common $k_{x}$ (marked by black dashed line).

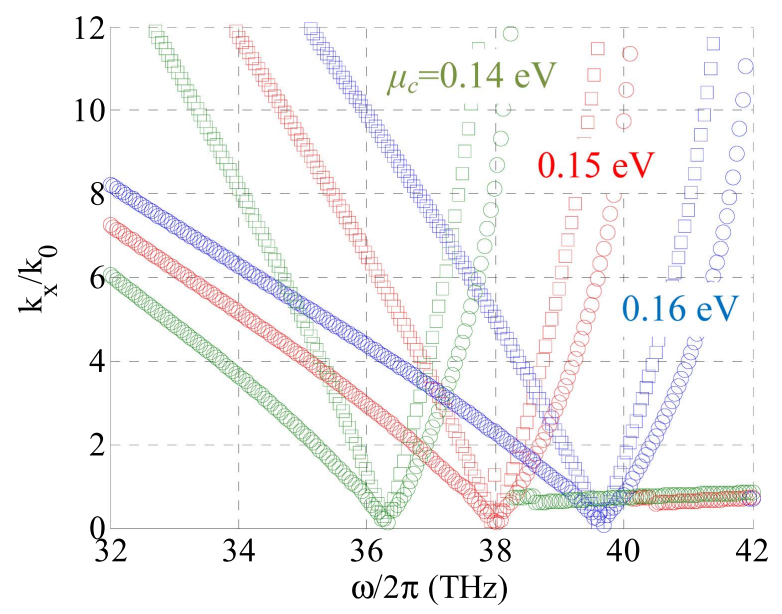

Figure 15. Representation of the solutions of (3.11) on the map of frequency $f$ and the real part of the normalised supported wavenumber along $x$ axis $\operatorname{Re}\left[k_{x}\right] / k_{0}$ for various doping of graphene $\mu_{c}$. Plot parameters: $\theta=25^{\circ}, d=20 \mathrm{~nm}, h=300 \mathrm{~nm}, \tau=10$ ps.

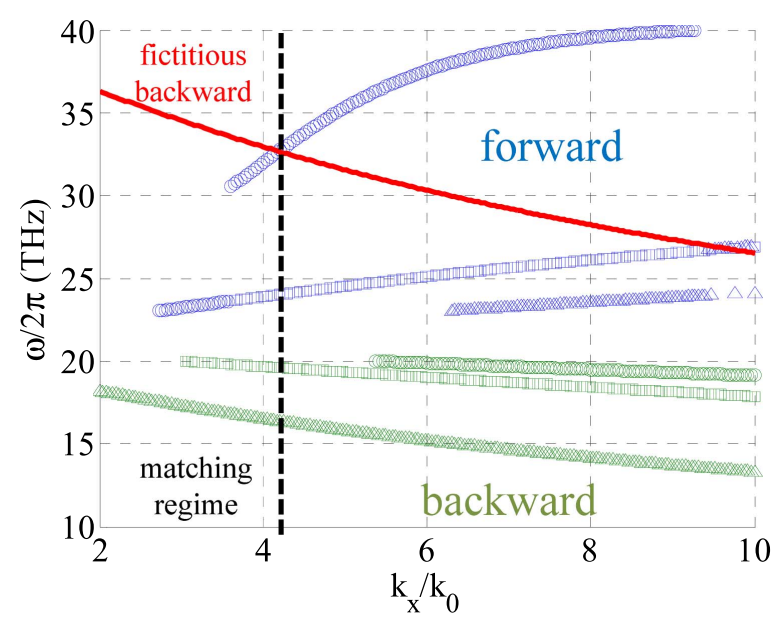

Figure 16. Representation of dispersion curves corresponding to three backward modes (green) and three forward modes (blue). The red line gives the curve of a (fictitious) backward wave dispersion relation. The black dashed line gives the wavenumber for matching

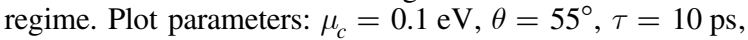
$d=20 \mathrm{~nm}$, and $h=300 \mathrm{~nm}$.

\section{Nonlinear stationary and non-stationary diffraction in active planar anisotropic hyperbolic metamaterial}

\subsection{Introduction}

In the present section, we consider nonlinear non-stationary diffraction in active planar anisotropic hyperbolic metamaterials [61-66]. Such a metamaterial is definitely periodical and multilayered. In the present section each a layer from which a metamaterial consists of ('elementary layers'), is considered to be as such an isotropic. All an anisotropy of a metamaterial as a whole will follow from the multilayered structure only and not from a properties of the medium 
included in separate layers. In this section, we will emphasise the following questions. (i) Rather general nonlinear evolution equation where only temporal dependence of an amplitude is supposed to be slow, and not spatial dependence. (ii) Hyperbolic metamaterial is considered as periodical media with repeating elementary cell with three layers inside each. In the coordinate frame $O Z$ axis is aligned perpendicularly to the boundaries of layers, $O X$ one is along the boundaries. Therefore, the tensor of the effective permittivity possesses only diagonal components. One of these layers (namely the third) is supposed to be active, and for the simplification, the approximation of the total compensation between the losses and gain is used in the present paper. (iii) We compare two approaches. Namely, one of them is based on the averaging over an unit cell, while the other one does not include sic an averaging. The first one is, in fact, metamaterial approach. The second one is more accurate than the metamaterial approach and is applicable for the mesoscale medium with the scales intermediate between metamaterials and photonic crystals. We would like to emphasise that these two approaches are rather complimentary, than contradictive to each other! In fact the more accurate approach without an averaging will be used, first of all, to justify the possibility of an application of the method of the averaging for the nonlinear active hyperbolic (metamaterial) media. Again, we emphasise that such a possibility is not evident as such for a nonlinear hyperbolic media even in the case when the metamaterial approximation is valid in the linear limit (or in the case of relatively small amplitude). It is very important in the context, that the metamaterial is nonlinear and active. In this paper we will consider only the case of total net gain/losses, in other words their exact mutual compensation. There is also another aspect of the comparison between the results of the above mentioned two approaches to the modelling of the nonlinear wave processes in a (layered) hyperbolic active metamaterial media: with and without an averaging. The point is that, in the active nonlinear media, stationary solution could not exist at all, at least for some specific set of parameters. Therefore, generally speaking, even an existence of a stationary solution (s) for some specific conditions specified below, will be investigated. To provide a possibility of such an investigation, the creation of the corresponding algorithms will be necessary. (iv) In the moderately nonlinear regime, a possibility of a formation of hot spots is investigated. This is exactly the case, when the investigation included a possibility of an existence of a stationary regime. To provide this, a proper algorithm have been developed, basing on the method of establishing stationary solution (asymptotically, as an evolution of the corresponding non-stationary solution) [67]. If we consider a propagation of the electromagnetic waves through the layer of a hyperbolic active nonlinear metamaterial with the finite width, such a propagation includes the reflections of two types. The first one is the reflections on the boundaries between the elementary layers. The second one is the reflection on the (two) boundaries between the metamaterial and surrounded medium (media), and the last will be considered as to be the semi-infinite and homogeneous, for the simplification. The following physical and methodological

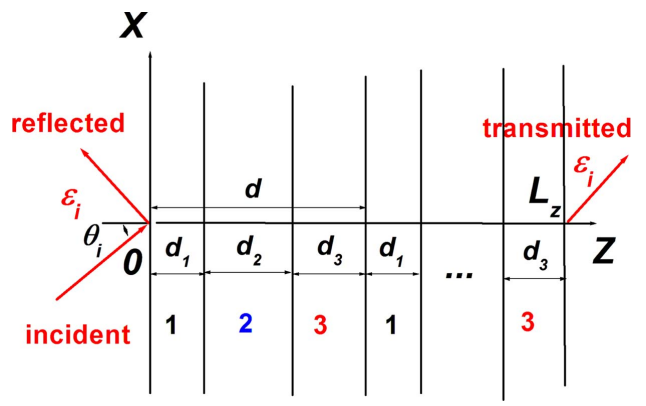

Figure 17. Geometry of the problem. The elementary cell includes 3 layers. Layer 1 possesses $\varepsilon_{L 1}<0$, layers 2,3 possess $\varepsilon_{L 2,3}>0$.

questions are addressed. (1) What should be the physical approximations and the form of the corresponding equations, necessary to describe such a wave propagation and search the corresponding physical effects? (2) Which interesting nonlinear effects and under which conditions are possible in a real active nonlinear metamaterial under a condition of the moderate nonlinearity (corresponding criteria of the moderate character of the nonlinearity will be clarified later)? The last question in the most general formulation is very non-trivial. The thicknesses of the elementary layers should be specified, when the results coincide, which are obtained from two approaches pointed above. Any real metamaterial includes finite losses or gain. In the present paper we will only start the consideration of these problems for active nonlinear hyperbolic medium, and use an approximation of totally compensation between gain and losses: the case of a medium with net zero losses/gain.

\subsection{Basic equations. Two approaches: with and without an everaging}

Consider the nonlinear propagation of electromagnetic waves in a bounded layered medium. There are 3 alternating elementary layers of thicknesses $d_{1}, d_{2}, d_{3}$, as seen in figure 17 .

Thus, the thickness of the elementary cell is $d=d_{1}+d_{2}+d_{3}$. Along $O Y$ axis the system is uniform. The propagation of nonlinear modulated waves with the components $E_{x}, H_{y}, E_{z}$ is investigated. The positive frequency components are $\sim \exp (-\mathrm{i} \omega t)$, where $t$ is time, $\omega>0$ is a circular frequency. Within each (isotropic) layer the dependence of the permittivity of the electric field is [68]:

$$
\begin{aligned}
& \varepsilon(z, x)=\varepsilon_{L}(z)+\frac{\alpha(z)|E|^{2}}{1+\gamma(z)|E|^{2}} ; \\
& |E|^{2} \equiv\left|E_{x}\right|^{2}+\left|E_{z}\right|^{2} .
\end{aligned}
$$

The model of the local saturating nonlinearity is used here [68]. Each elementary layer is assumed as uniform, so $\varepsilon_{L}, \alpha, \gamma$ are step-like functions of $z$. Generally $\varepsilon_{L}$ and $\alpha$ are complex, because the cases of possible dissipation and active media are investigated. The coefficient of the saturation of the nonlinearity is assumed as real and positive: $\gamma>0$. 
The Maxwell equations are:

$$
\nabla \times \vec{H}=\frac{1}{c} \frac{\partial \vec{D}}{\partial t} ; \nabla \times \vec{E}=-\frac{1}{c} \frac{\partial \vec{H}}{\partial t} .
$$

Below the lengths are normalised to $l_{n}=1 \mu \mathrm{m}$, time is normalised to $t_{n}=l_{n} / c$. The relations between the components of the electromagnetic field are:

$E_{x}=-\frac{\mathrm{i} \beta}{\omega} \frac{\partial H}{\partial z} \mathrm{e}^{-\mathrm{i} \omega t}, H_{y}=H \mathrm{e}^{-\mathrm{i} \omega t}, E_{z}=\frac{\mathrm{i} \beta}{\omega} \frac{\partial H}{\partial x} \mathrm{e}^{-\mathrm{i} \omega t} ;$

(1) Let us consider the first approach. The equation for the slowly varying amplitude of the magnetic field $H$ is:

$\frac{\partial H}{\partial t}-\frac{\mathrm{i}}{2 \omega} \frac{\partial}{\partial x}\left(\beta \frac{\partial H}{\partial x}\right)-\frac{\mathrm{i}}{2 \omega} \frac{\partial}{\partial z}\left(\beta \frac{\partial H}{\partial z}\right)-\frac{\mathrm{i} \omega}{2} H=0$.

Note that equation (4.4) is written for each elementary layer, and each of them is isotropic as such for the used model of a hyperbolic metamaterial. Standard boundary conditions of the continuity of $E_{x}, H_{y}$ at the boundaries $z=0$ and $z=L$ between the layer of the metamaterial and surrounding medium and between all elementary layers included into the metamaterial (figure 17) are applied. Generally $H(z, x$, $t$ ) is slowly varying in time only, whereas the dependencies on $z$ and $x$ are arbitrary. Both direct and reflected waves in each layer are taken into account here. In equation (4.4) the inverse permittivities $\beta(z, x)$ depend on both coordinates, especially on $x$ due to nonlinearity, and are step-like functions on $z$.

The thickness of layered medium is $L_{z}$, so it is localised within the interval $0<z<L_{z}$. At $z<0$ and $z>L_{z}$ there are linear media with the real permittivity $\varepsilon_{i}$. At $z<0$ there are an incident wave with the incidence angle $\theta_{i}$ and reflected one. At $z>L_{z}$ there exists the transmitted wave only.

At $z<0$ the magnetic field is the sum of the incident wave and reflected one:

$$
\begin{aligned}
H= & A_{i}(z, x, t) \exp \left(\mathrm{i} k_{i x} x+\mathrm{i} k_{i z} z\right) \\
& +A_{r}(x) \exp \left(-\mathrm{i} k_{i x} x+\mathrm{i} k_{i z} z\right) \\
k_{i x}= & k_{i} \sin \theta_{i} ; \quad k_{i z}=k_{i} \cos \theta_{i} ; k_{i} \equiv \omega \varepsilon_{i}^{1 / 2} .
\end{aligned}
$$

The slowly varying amplitudes $A_{i, r}$ satisfy the following equations:

$$
\begin{aligned}
\frac{\partial A_{i}}{\partial t}+v_{z} \frac{\partial A_{i}}{\partial z}+v_{x} \frac{\partial A_{i}}{\partial x} \approx 0 ; \frac{\partial A_{r}}{\partial t}-v_{z} \frac{\partial A_{r}}{\partial z} \\
+v_{x} \frac{\partial A_{r}}{\partial x} \approx 0 ; v_{x} \equiv \beta_{i} \sin \theta_{i} ; v_{x} \equiv \beta_{i} \cos \theta_{i} \\
\beta_{i} \equiv \frac{1}{\varepsilon_{i}} .
\end{aligned}
$$

The boundary conditions at $z=0$ can be written down as:

$$
\begin{aligned}
& \left.\left(A_{i}+A_{r}\right)\right|_{(z=-0)} \exp \left(\mathrm{i} k_{i x} x\right)=\left.H\right|_{(z=+0)} ; \\
& \left.\beta_{i}\left(\mathrm{i} k_{i z}\left(A_{i}-A_{r}\right)+\frac{\partial A_{i}}{\partial z}+\frac{\partial A_{r}}{\partial z}\right)\right|_{(z=-0)} \exp \left(\mathrm{i} k_{i x} x\right) \\
& \quad=\left.\beta_{1} \frac{\partial H}{\partial z}\right|_{(z=+0)} .
\end{aligned}
$$

After expression of the derivatives $\partial A_{i} / \partial z, \partial A_{r} / \partial z$ from (4.6), one can get the following approximate boundary condition for $H$ at $z=0$ :

$$
\begin{aligned}
& \left(1+\frac{k_{i x} v_{x}}{k_{i z} v_{z}}\right) \cdot H-\frac{\mathrm{i} \beta_{1}}{\beta_{i} k_{i z}} \frac{\partial H}{\partial z}+\left.\frac{\mathrm{i}}{k_{i z} v_{z}}\left(\frac{\partial H}{\partial t}+v_{x} \frac{\partial H}{\partial x}\right)\right|_{(z=+0)} \\
& \left.\approx 2\left[A_{i}+\frac{\mathrm{i}}{k_{i z} v_{z}}\left(\frac{\partial A_{i}}{\partial t}+v_{x} \frac{\partial A_{i}}{\partial x}\right)\right]\right|_{(z=-0)} \exp \left(\mathrm{i} k_{i x} x\right)
\end{aligned}
$$

Because at $z>L_{z}$ the outgoing wave exists only, at $z=L_{z}$ the corresponding boundary condition is

$$
\begin{aligned}
\left(1+\frac{k_{i x} v_{x}}{k_{i z} v_{z}}\right) \cdot H+\frac{\mathrm{i} \beta_{3}}{\beta_{i} k_{i z}} \frac{\partial H}{\partial z} \\
+\left.\frac{\mathrm{i}}{k_{i z} v_{z}}\left(\frac{\partial H}{\partial t}+v_{x} \frac{\partial H}{\partial x}\right)\right|_{\left(z=L_{z}-0\right)} \approx 0
\end{aligned}
$$

(2) Also the second approach is applied where the averaged values of the permittivity and therefore a consideration of the hyperbolic metamaterial as effectively continuous medium, are used. Accurately speaking, this approach is applicable, when the typical width of the layers is much less than the wavelength in the medium. Nevertheless we will compare the results of both approaches for the values of the widths of the layers lying in the range from 0.1 to 0.25 of the wavelength. It is assumed that the elementary layers 2, 3, are nonlinear, whereas the 1st one is linear. The non-dimensional units are used with $c=1$. Within the averaged medium we have in each point of it:

$$
E_{x}=-\frac{\mathrm{i} \beta_{x}}{\omega} \frac{\partial H}{\partial z} ; E_{z}=\frac{\mathrm{i} \beta_{z}}{\omega} \frac{\partial H}{\partial x} .
$$

Here $E_{x}, E_{z}$ are averaged values of the components of the electric field: $E_{x} \equiv\left\langle E_{x}\right\rangle, E_{z} \equiv\left\langle E_{z}\right\rangle$. In this approach the equation for the slowly varying amplitude $H(z, x, t)$ is:

$$
\frac{\partial H}{\partial t}-\frac{\mathrm{i}}{2 \omega} \frac{\partial}{\partial x}\left(\beta_{z} \frac{\partial H}{\partial x}\right)-\frac{\mathrm{i}}{2 \omega} \frac{\partial}{\partial z}\left(\beta_{x} \frac{\partial H}{\partial z}\right)-\frac{\mathrm{i} \omega}{2} H=0 .
$$

The amplitude $H(z, x, t)$ is slowly varying with respect to time $t$ only, as in equation (4.4). The formulas for the averaged 
components of the inverse permittivity are:

$$
\begin{aligned}
& \beta_{z}=\beta_{1} \frac{d_{1}}{d}+\beta_{2} \frac{d_{2}}{d}+\beta_{3} \frac{d_{3}}{d}, \beta_{1,2,3}=\frac{1}{\varepsilon_{1,2,3}} ; \\
& \varepsilon_{x}=\varepsilon_{1} \frac{d_{1}}{d}+\varepsilon_{2} \frac{d_{2}}{d}+\varepsilon_{3} \frac{d_{3}}{d}, \beta_{x}=\frac{1}{\varepsilon_{x}} .
\end{aligned}
$$

These formulas are applied in each point of the averaged medium, where the values of $E_{x}, E_{z}$ are computed. Here $d_{1,2,3}$ are the thicknesses of the elementary layers, $d=d_{1}+$ $d_{2}+d_{3}$. In (2) only the ratios $d_{1,2,3} / d$ are important but not their absolute values. Respectively, it is supposed that the change of the field components $E_{x, z}, H_{y}$, as well as of the values $\beta_{x, z}$ along the layers (in the direction $x$ ) happen on the lengths, much larger than the thicknesses of the layers $d_{1,2,3}$. The approximation of this layers is used here. The nonlinear permittivities of the elementary layers 2, 3 are calculated as:

$$
\begin{aligned}
& \varepsilon_{2}=\varepsilon_{2 L}+\frac{\alpha_{2} \cdot\left(\left|E_{2 x}\right|^{2}+\left|E_{2 z}\right|^{2}\right)}{1+\gamma_{2} \cdot\left(\left|E_{2 x}\right|^{2}+\left|E_{2 z}\right|^{2}\right)} ; \\
& \varepsilon_{3}=\varepsilon_{3 L}+\frac{\alpha_{3} \cdot\left(\left|E_{3 x}\right|^{2}+\left|E_{3 z}\right|^{2}\right)}{1+\gamma_{3} \cdot\left(\left|E_{3 x}\right|^{2}+\left|E_{3 z}\right|^{2}\right)} .
\end{aligned}
$$

Here $E_{2 x}$ etc are the components of the electric field in the corresponding elementary layers near the specific point within the averaged medium. There is the problem of the correspondence between the averaged values $E_{x}, E_{z}$ and $E_{2 x}$, $E_{3 x}, E_{2 z}, E_{3 z}$ within the elementary layers. Because the tangential component of the electric field is continuous in the layered medium, one can write down:

$$
E_{2 x}=E_{3 x}=E_{x} .
$$

The normal component of the electric induction is also continuous:

$$
D_{2 z}=D_{3 z}=\left\langle D_{z}\right\rangle \equiv \frac{E_{z}}{\beta_{z}} .
$$

It is follows from (5) that

$$
E_{2 z}=\beta_{2} D_{2 z}=\frac{\beta_{2}}{\beta_{z}} E_{z} ; E_{3 z}=\beta_{3} D_{3 z}=\frac{\beta_{3}}{\beta_{z}} E_{z} .
$$

The formulas (4.10)-(4.15) are used jointly, so several iterations should be applied. Note that in distinction to equation (4) used in the first considered approach, equation (4.11) describes the whole metamaterial as a continuous medium. Therefore the boundary conditions between the elementary layers included in the metamaterial (figure 17) are not applied for the equation (4.4). Nevertheless the boundary conditions at the boundaries $z=0$ and $z=L$ between the layer of the metamaterial and surrounding medium are still necessary and applied. The last boundary conditions have the form similar to (4.9).

Note the following concerning the physical sense of the averaging, formulas (4.10)-(4.15). The presence in the metamaterial, consisted of the periodically alternation isotropic layers, of the anisotropy, which is evident from the formulas (4.11), has a clear physical sense. Let us consider first a linear media. In this case formulas (4.11) have been derived accurately, (this derivation is not presented here) using the consideration of the periodical media with the farther approximation of thin layers. Qualitatively, the description of the anisotropy of the hyperbolic metamaterial may be drawn from the equivalent transmission line, describing a planar multilayered metamaterial $[69$, chapter 7 , paragraph 2 , formulas (7a) and (7b)]. In accordance with this approach, an averaged transverse field components $\left(E_{x}, H_{y}\right.$ in our case) would be determined through an average value of the effective impedance, which is proportional to $\left\langle\varepsilon_{x x}\right\rangle$, where the brackets $\langle\ldots\rangle$ mean a proper averaging (in our case by the period of the structure) of the value placed inside the brackets. From the other hand, as it follows from [69, chapter 7 , paragraph 2, formula (4)], an averaged longitudinal field component, in our case $E_{z}$, would be determined through the value proportional to $\left\langle\varepsilon_{z z}^{-1}\right\rangle$. For the hyperbolic metamaterial, based on the isotropic alternating layers, in each, $\varepsilon_{x x}=\varepsilon_{z z}$, while the signs of $\varepsilon_{x x}$ alternate from layer to layer belonging to each cell of the structure. Providing that $\left\langle\varepsilon_{x x}\right\rangle>0$, it is easy to see that $\left\langle\varepsilon_{x x}^{-1}\right\rangle=\left\langle\varepsilon_{z z}^{-1}\right\rangle<0$. Therefore we get hyperbolic uniaxial metamaterial with the opposite signs of the diagonal tensors of the dielectric permittivity. In the nonlinear case, of course, the periodicity of the medium, which is the basis of an averaging, disappears. Nevertheless we still use the relations (4.11) in the same form, as that for the linear case, phenomenologically, accounting for a contribution of the nonlinearity into the values $\varepsilon_{2,3}$ (formulas (4.12)) and, respectively to $\beta_{1,2,3}, \varepsilon_{x, z}$ (formulas (4.11)), as it is described above. This is one of the reasons why the comparison between the accurate approach without an averaging (see equation (4.4)) with the metamaterial approximation of the continuous media (equation $(4.10 b)$ ) is really necessary and important.

Equation (4.4) added by boundary conditions has been solved by the finite differences method where the operator factorisation, or the method of Douglas-Rachford [67], has been applied namely equation (4.4) is rewritten symbolically as:

$$
\begin{aligned}
& \frac{\partial H}{\partial t}+\hat{L}_{1} H+\hat{L}_{2} H=0, \\
& \text { where } \hat{L}_{1} H \equiv-\frac{\mathrm{i}}{2 \omega} \frac{\partial}{\partial z}\left(\beta \frac{\partial H}{\partial z}\right)-\frac{\mathrm{i} \omega}{2} H, \\
& \hat{L}_{2} H \equiv-\frac{\mathrm{i}}{2 \omega} \frac{\partial}{\partial x}\left(\beta \frac{\partial H}{\partial x}\right) .
\end{aligned}
$$

The following notation is used:

$$
\chi^{p+1} \equiv \frac{H^{p+1}-H^{p}}{\tau}
$$

Here $\tau$ is the step for time $t, t^{p} \equiv p \cdot \tau, p=0,1,2 \ldots ; H^{p} \equiv$ $H\left(t^{p}\right)$. And equations (4.16)-(4.13) can be represented as:

$$
\begin{aligned}
& \left(1+\tau \hat{L}_{2}\right) \chi^{p+1 / 2}=-\left(\hat{L}_{1}+\hat{L}_{2}\right) H^{p} \\
& \left(1+\tau \hat{L}_{1}\right) \chi^{p+1}=\chi^{p+1 / 2} ; \\
& H^{p+1}=H^{p}+\tau \chi^{p+1} .
\end{aligned}
$$


The boundary conditions (4.8) and (4.9) are also rewritten in terms of $\chi^{p+1}$. The operators $\hat{L}_{1,2}$ are approximated by finite differences $[67,70]$. The inverse permittivity $\beta$ is a piecewisecontinuous function of $z$, so the values of $\beta$ are approximated between the nodes for $H$ : if the values of $H$ in the nodes are $H_{j} \equiv H\left(z_{j}\right) \equiv H\left(j \cdot h_{z}\right), j=0,1,2 \ldots$, then $\beta_{j+1 / 2} \equiv$ $\beta\left((j+1 / 2) \cdot h_{z}\right)$. Each distance $d_{1,2,3}$ includes integer numbers of spatial steps $h_{z}$. To take into account the nonlinearity, the iterations have been applied. This method is unconditionally stable.

The system is limited in $x$-direction, $0 \leqslant x \leqslant L_{x}$, so the boundary conditions at $x=0$ and $x=L_{x}$ are $H=0$.

The boundary conditions (4.8) and (4.9) have been approximated by finite differences too. They are applied at the second fractional step in (4.18), to compute $\chi^{p+1}$. The derivatives $\partial \chi^{p+1} / \partial x$ are calculated in the positive direction of $x$, because $v_{x}>0$. In the point $x_{l} \equiv l \cdot h_{x}$ the approximation is $\partial \chi^{p+1} / \partial x \approx\left(\chi_{l}^{p+1}-\chi_{-1}^{p+1}\right) / h_{x}$. This approximation makes possible to calculate $\chi^{p+1}$ from smaller values of $x$ to higher ones: $l=1,2,3, \ldots$, and to use for computing $\chi_{l}^{p+1}$ the value of $\chi_{l-1}^{p+1}$, which has been just calculated.

The implicit-explicit methods, like Peaceman-Rachford one [67], do not provide good stability in our nonlinear case, as our simulations demonstrated. The schemes like splitting with respect to physical factors, or the summatory approximation, require small temporal steps and therefore practically unusable here.

The incident wave is assumed as a beam bounded in $x$ direction:

$$
A_{i}(z=0, x, t)=A_{0} \exp \left(-\left(\frac{x-x_{1}}{x_{0}}\right)^{2}\right) \tanh \left(\frac{t}{t_{0}}\right)
$$

The temporal dependence is tanh-like and the maximum amplitude of the incident wave tends to $A_{0}$ at the boundary $z=0$. Below the established values of the electromagnetic field are presented. As it will be shown below, strongly nonlinear phenomena, namely hot spot formation is possible for the present system with the corresponding parameters. This is the case, when a possibility of stationary solution is not evident beforehand, and to prove such a possibility, the method of establishing (steady-state solution) and, respectively, the initial-boundary condition (4.19) are quite adequate.

\subsection{Details of the structure and requirements for materials}

4.3.1. Details of the structure. The simulations have been done for hyperbolic media. For linear EM waves the parameters of the elementary layers are chosen to get $\varepsilon_{x}^{\prime}>0, \varepsilon_{x}^{\prime}<0$, where $\varepsilon^{\prime} \equiv \operatorname{Re}(\varepsilon)$. The medium 1 is with $\varepsilon_{L 1}^{\prime}<0$, ones 2 and 3 are with $\varepsilon_{L 1}^{\prime}>0$. The hyperbolic media possess the properties, which are important both for theoretical and practical views. Below it is assumed that the real parts of the media 2, 3 are equal: $\varepsilon_{L 2}^{\prime}=\varepsilon_{L 3}^{\prime}$. The anisotropy is neglected.

4.3.2. Requirements for materials. The materials for the elementary layers should satisfy the following requirements: $\left|\varepsilon_{L 1}^{\prime}\right|<\varepsilon_{L 2}^{\prime}$; the dissipation within each layer should be smaller as possible.

In the near-infrared and visible optical range (wavelengths $\lambda_{0}=0.5-2 \mu \mathrm{m}, \omega=5 \times 10^{14}-4 \times 10^{15} \mathrm{~s}^{-1}$ ) the medium 1 can be metallic of high conductivity or semimetallic, like $\mathrm{Ag}, \mathrm{Au}, \mathrm{Cu}, \mathrm{Bi}$. The linear effective permittivity is:

$$
\varepsilon_{l 1}=\varepsilon_{\text {lattice }}-\frac{\omega_{p}^{2}}{\omega(\omega+\mathrm{i} \nu)} ; \omega_{p}^{2} \equiv \frac{4 \pi e^{2} n_{0}}{m^{*}} .
$$

Here $\omega_{p}$ is the plasma frequency, $n_{0}$ is the electron concentration, $m^{*}$ is the effective electron mass, $\nu$ is the electron collision frequency.

The critical parameters are the electron concentration $n_{O}$ and the collision frequency. It should be preferably $n_{0}=10^{21}-2 \times 10^{22} \mathrm{~cm}^{-3}$, to provide the negative effective permittivity in the corresponding layer(s), as it is required for the hyperbolic metamaterial, in the optical range of moderate absolute values $\varepsilon_{L 1}^{\prime}=-3$ to -10 . Namely, the effective permittivity is negative due to the electron plasma, thus, $0.2 \omega_{p}$ $<\omega<0.5 \omega_{p}$. The collision frequency should be relatively small $\nu \leqslant 10^{13} \mathrm{~s}^{-1}$.

The layers 2, 3 should be dielectrics with high (with a positive real part) permittivity in the optical range and low losses (or even active, i.e. to provide amplification), like $\mathrm{Hf}_{2} \mathrm{O}_{5}, \mathrm{Ta}_{2} \mathrm{O}_{5}, \mathrm{Al}_{2} \mathrm{O}_{3}, \varepsilon_{L 2}^{\prime} \geqslant 10$.

In the $\mathrm{THz}$ range (wavelengths $\lambda_{0}=0.5-0.03 \mathrm{~mm}$, or $\left.\omega=10^{12}-6 \times 10^{13} \mathrm{~s}^{-1}\right)$ the narrow-gap semiconductors $n$ InSb, $n$-InAs, $n-\mathrm{Cd}_{1-x} \mathrm{Hg}_{x} \mathrm{Te}$ are perspective. The frequency of EM wave should satisfy the inequality $\omega<E_{g} / \hbar$, where $E_{g}=0.1-0.4 \mathrm{eV}$ is the forbidden gap for narrow-gap semiconductors. These semiconductors possess small effective electron masses $m^{*}=(0.002-0.02) m_{e}$ and low collision frequencies $\nu=\left(10^{11}-3 \times 10^{11}\right) \mathrm{s}^{-1}$ at moderate doping levels $n_{0}=\left(10^{15}-10^{16}\right) \mathrm{cm}^{-3}$. As the layers 2, 3, the dielectrics like $\mathrm{TiO}_{2}, \mathrm{MoO}_{2}, \mathrm{SrTiO}_{3}, \mathrm{LiNbO}_{3}$ with high permittivities $\varepsilon_{L 2}^{\prime} \geqslant 20$ can be used. Also $\mathrm{SrTiO}_{3}$ and $\mathrm{LiNbO}_{3}$ possess high dielectric cubic nonlinearity of the negative signs.

In the optical range the unity of unidimensional amplitude of the electric field can be estimated as $1 \mathrm{GW} \mathrm{cm}^{-2}$ for the intensity. In $\mathrm{THz}$ range the unity is estimated as $10 \mathrm{MW} \mathrm{cm}^{-2}$ for dielectrics of high nonlinearity.

It is demonstrated below that the nonlinear behaviour of EM waves within the hyperbolic medium depends essentially on the sign of cubic nonlinearity.

The surrounding media at $z<0$ and $z>L_{z}$ possess the permittivity $\varepsilon_{i}$ matched to the hyperbolic medium in the linear case. Namely, the value of $\varepsilon_{i}$ has been chosen to provide zero reflection coefficient at $z=0$ in the case of the incidence of the plane wave at the incidence angle $\theta_{i}$. 
In the experimental realisation of hyperbolic media the essential problem is the linear dissipation, because usually the elementary layer 1 is metallic or semimetallic. Therefore, to observe various wave phenomena there it is rather better to provide a compensation of this dissipation, and the elementary layer 3 should be active, where $\epsilon_{L 3}^{\prime \prime}<0$. Below namely the case of the compensated dissipation is considered, where all linear permititvities $\varepsilon_{L}$ and nonlinear coefficients $\alpha$ are assumed as real. The results of simulations are tolerant to changes of the lengths and the widths of the hyperbolic medium.

\subsection{Results of simulations}

All of the computations under this heading have been performed with original programs created with the use of Lahey/Fujitsu Fortran 95, licenced as CIICAp, UAEM in Mexico. In the linear case, i.e. absence of nonlinearity, the main attention is given to comparison of the simulations of wave beam propagation within the framework of direct simulations without averaging and for averaged media (figure 18). During the simulations without an averaging, it is used that the values in the brackets in the third terms in equations (4.4) and (4.10b) is continuous on the interface between the layers of the hyperbolic medium (because they are proportional to the tangential components of the corresponding electric fields, in accordance with equations (4.3) and $(4.10 b)$ ). The results of simulations have been practically the same when the thicknesses $d_{j}$ of the elementary layers should satisfy the inequalities $d_{j} \cdot \omega / c\left(\left|\varepsilon_{L j}\right|\right)^{1 / 2} \leqslant 0.05$. When, for instance, the frequency of EM wave is $\omega=2 \times 10^{15} \mathrm{~s}^{-1}$, the permittivities are $\varepsilon_{L 1}=-10, \varepsilon_{L 2,3}=20$, the thicknesses of the elementary layers should be $d_{2,3} \leqslant 10 \mathrm{~nm}, d_{1} \leqslant 20 \mathrm{~nm}$.

The used parameters are $\varepsilon_{L 1}=-10, \varepsilon_{L 2,3}=20, L_{z}$ $=50 \mu \mathrm{m}, L_{x}=20 \mu \mathrm{m}$, half-widths of incidents beam are $x_{0}=1-3 \mu \mathrm{m}, \omega=(1-30) \times 10^{14} \mathrm{~s}^{-1}$, incidence angles $\theta_{i}$ $=(0-30)^{\circ}$. The thicknesses of the elementary layers are chosen as $d_{1,2,3}=5-20 \mathrm{~nm}$. The typical results are given in figure 18. It is seen that the results of non-averaged simulations, equation (4.4), and within the framework of the averaged medium, equation $(4.10 b)$, coincide. In figure 18 the incident beam possesses the incidence angle $\theta_{i}=30^{\circ}, x$ component of the group velocity of the incident beam is positive and directed upwards. The $x$-component of the group velocity of the refracted wave within the hyperbolic medium at $x>0$ is negative and is directed downwards. Thus, the hyperbolic medium possesses the negative refraction.

In the nonlinear case, the layers 2, 3 are assumed to be nonlinear, whereas 1 is linear. Two cases are considered: $\alpha>0$ (figure 19) and $\alpha<0$ (figures 20 and 21). In the first case the input beam is subject to essential nonlinear diffraction. The second case is much more interesting. Within the hyperbolic media the hotspots are formed (near $x \approx 13 \mu \mathrm{m}, z \approx 27 \mu \mathrm{m}$ figure 20 and $x \approx 12 \mu \mathrm{m}, z \approx 35 \mu \mathrm{m}$, figure 21) where the EM energy concentrates.

Note that (1) while beam width increases in two times, the intensity in the peak of hot spot increases in $\sim 1.5$ times (compare figure 21 with figure 20), and (2) for the input beam width equal to $2 \mu \mathrm{m}$, the area of the hotspots are of order of $2 \mu \mathrm{m}^{2}$ (figure 21).
From figures 19-21 one can see that the nonlinearity is moderate there and the results of simulations are practically the same both in the direct simulations, equation (4.4), and with the averaged permittivities, equation (4.10b). The sizes of the hotspots in figures 20 and 21 are $2-3 \mu \mathrm{m}$, i.e. 3-5 wavelengths calculated for the averaged permittivity $\lambda=\lambda_{0} /\left(\varepsilon_{x}^{\prime}\right)^{1 / 2} \approx 0.4 \mu \mathrm{m}$. The moderate character of the nonlinearity is also determined by the following extra requirement, if the hotspots are under consideration. Namely, a typical size of the hotspots should be of the order of at least few, say five thicknesses of the elementary layers included into the metamaterial. Such a condition is satisfied for the present simulations.

\subsection{Limiting case of the stationary NSE}

Equation (4.11) can be reduced to the stationary NLSE [67] in the case of the moderate nonlinearity. NSE has the structure:

$$
K_{z} \frac{\partial U}{\partial \tilde{z}}+K_{x} \frac{\partial U}{\partial \tilde{x}}+\mathrm{i} G \frac{\partial^{2} U}{\partial \tilde{x}^{2}}+\mathrm{i} N F\left(|U|^{2}\right) U=0 .
$$

The wave amplitude $U(\tilde{z}, \tilde{x})$ is slowly varying with respect to $\tilde{z}$. The coefficients $K_{x, z}, N$, and the function $F$ can be expressed through the parameters of the hyperbolic medium and the frequency. Equation (4.21) has been written down in the rotated coordinate frame $\tilde{X} O \tilde{Z}$, where $O \tilde{Z}$ axis is aligned along the group velocity within the hyperbolic medium. The group velocity $\boldsymbol{V}_{\boldsymbol{g}}$ is obtained from the linear dispersion equation:

$$
\begin{aligned}
& D\left(k_{z}, k_{x}, \omega\right)=k_{z}{ }^{2} \beta_{x}+k_{x}{ }^{2} \beta_{z}-\omega^{2}=0, \\
& V_{g x} \equiv-\left(\frac{\partial D}{\partial k_{x}}\right) \cdot\left(\frac{\partial D}{\partial \omega}\right)^{-1}=\frac{k_{x} \beta_{z}}{\omega}<0, V_{g z} \\
& \quad \equiv-\left(\frac{\partial D}{\partial k_{z}}\right) \cdot\left(\frac{\partial D}{\partial \omega}\right)^{-1}=\frac{k_{z} \beta_{x}}{\omega}>0, \\
& k_{x}, k_{z}, \omega, \beta_{x}>0, \beta_{z}<0 .
\end{aligned}
$$

Here the frequency dispersion of the components of the inverse permittivity $\beta_{x, z}$ is neglected. When the frequency dispersion is taken into account, the signs of the components of the group velocity preserve.

Therefore, under enough small thicknesses of the elementary layers in the case of the moderate nonlinearity the dynamics of the nonlinear beams in the hyperbolic medium can be described by NSE, where one-directional propagation of EM wave is considered.

In the case of the propagation along $O Z$, i.e. perpendicularly to the boundaries of the layers, the structure of stationary NSE is standard [68]:

$$
\begin{aligned}
& \frac{\partial D}{\partial k_{z}} \frac{\partial U}{\partial z}-\mathrm{i} \frac{\partial D}{\partial\left(k_{x}^{2}\right)} \frac{\partial^{2} U}{\partial x^{2}}-\mathrm{i} \frac{d_{1}+d_{2}}{d} \omega^{2} \alpha|U|^{2} U=0 \\
& D\left(k_{z}, k_{x}, \omega\right)=k_{z}^{2} \beta_{x}+k_{x}^{2} \beta_{z}-\omega^{2}=0, \\
& \frac{\partial D}{\partial k_{z}}=2 k_{z} \beta_{x}>0, \frac{\partial D}{\partial\left(k_{x}^{2}\right)}=\beta_{z}<0 .
\end{aligned}
$$

Equation (4.23a) is written down in the case of moderate nonlinearity. Here $D=0$ is the linear dispersion equation for EM waves in the hyperbolic medium. Equation (4.23a) can be 


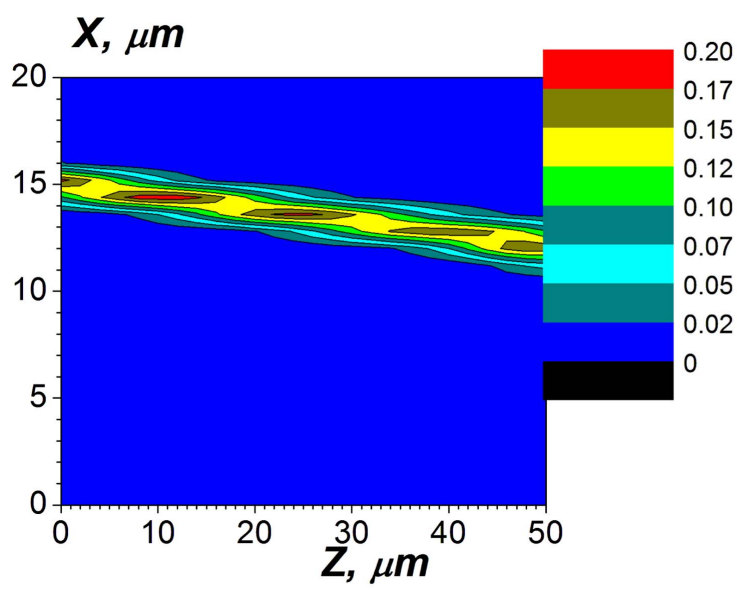

a)

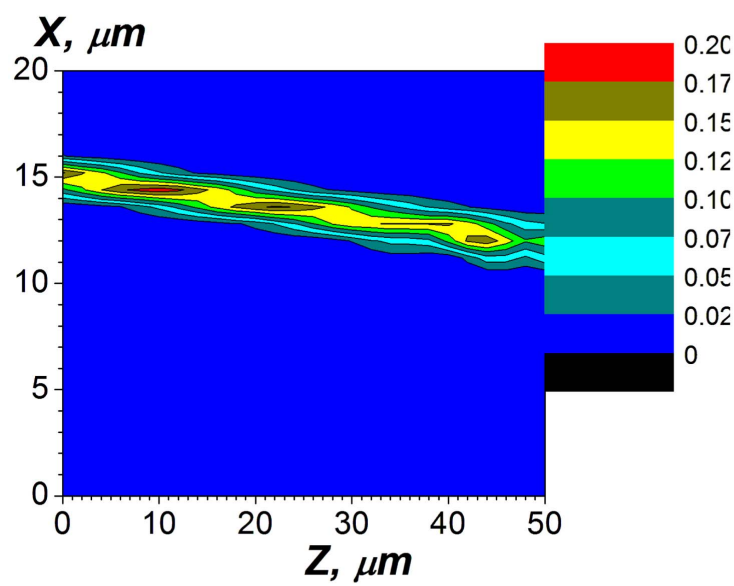

c)

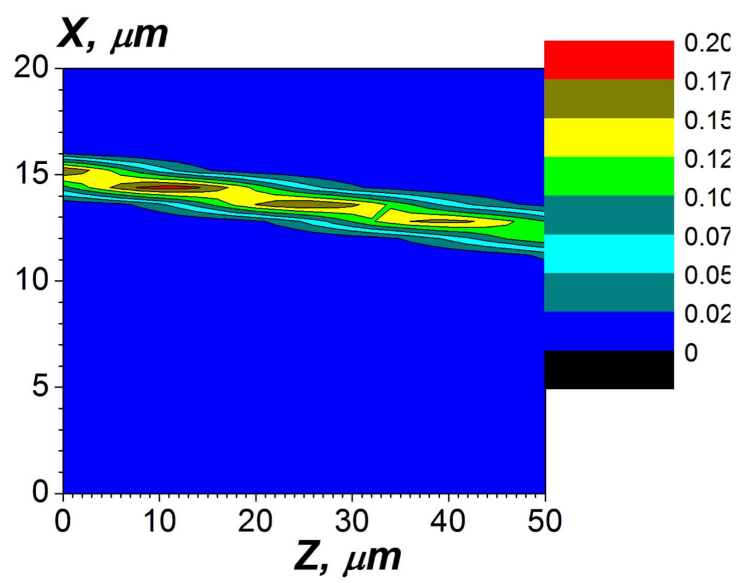

e)

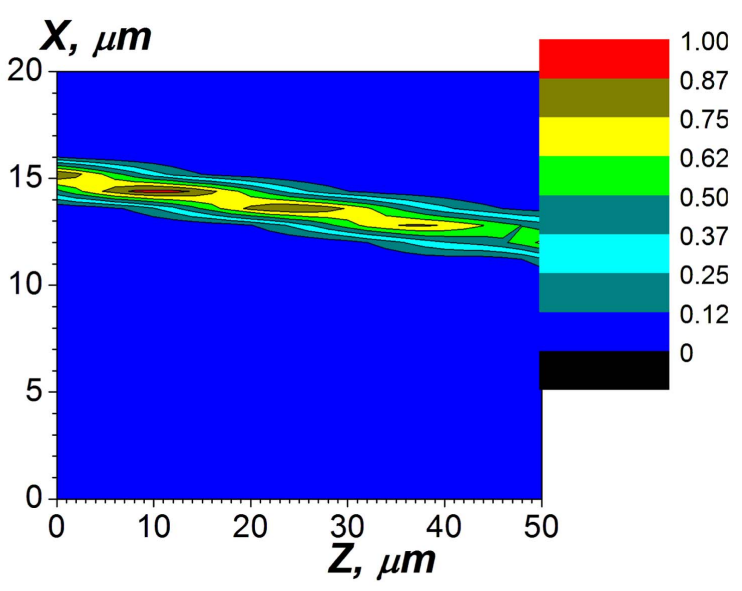

b)

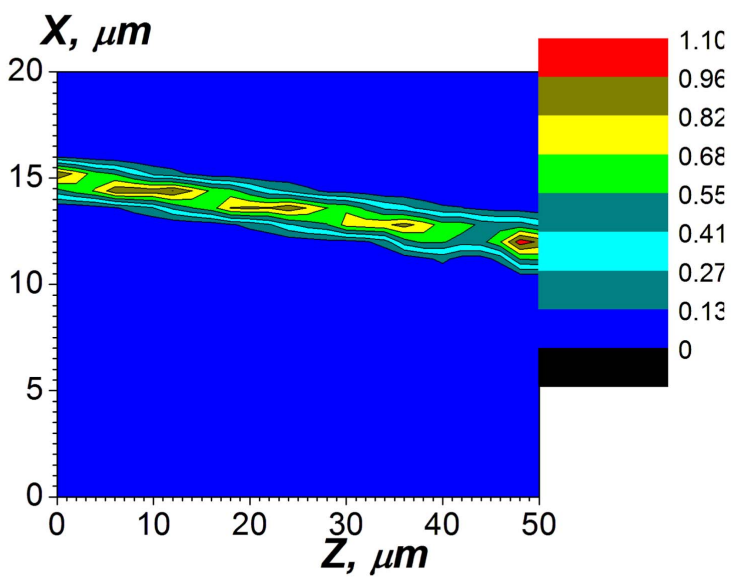

d)

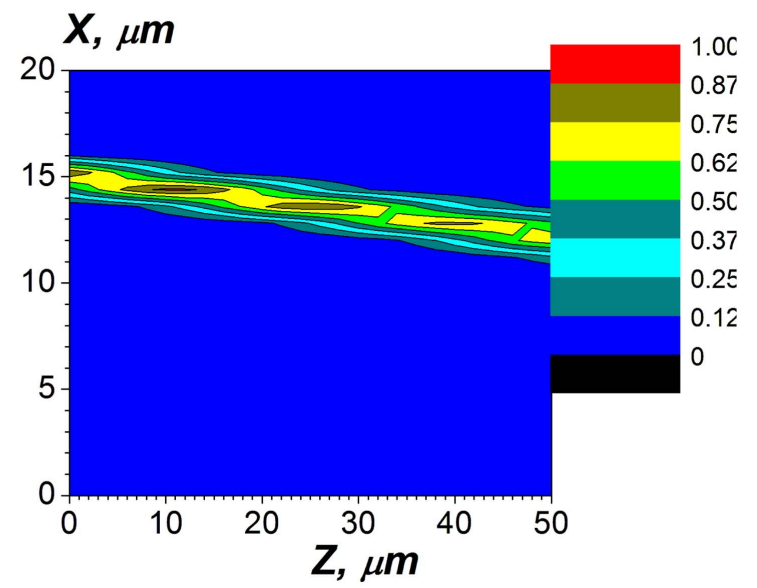

f)

Figure 18. Propagation of linear waves. The circular frequency is $\omega=6 \pi \times 10^{14} \mathrm{~s}^{-1}\left(\lambda_{0}=1 \mu \mathrm{m}\right)$. Parts (a), (b) are $|E|^{2}$ and $|H|^{2}$ for the thicknesses of elementary layers $d_{1}=7.5 \mathrm{~nm}, d_{2}=d_{3}=3.75 \mathrm{~nm}$. Parts (c), (d) are the same, but $d_{1}=15 \mathrm{~nm}, d_{2}=d_{3}=7.5 \mathrm{~nm}$. Parts (e), (f) are the same, but the simulations are within the framework of averaged permittivities. For the chosen parameters the averaged permittivities are $\varepsilon_{x}$ $=5, \varepsilon_{z}=-40$; the permittivity of the contacting medium is $\varepsilon_{I}=4.203$. The half-width of the incident beam is $x_{0}=1 \mu \mathrm{m}$.

rewritten in the equivalent manner:

$$
\frac{\partial U}{\partial z}-\mathrm{i} \frac{\beta_{z}}{2 k_{z} \beta_{x}} \frac{\partial^{2} U}{\partial x^{2}}-\mathrm{i} \frac{d_{1}+d_{2}}{d} \frac{\omega^{2} \alpha}{2 k_{z} \beta_{x}}|U|^{2} U=0 .
$$

One can see that in the case of the negative cubic nonlinearity $\alpha<0$ the signs of the diffraction and nonlinear coefficients coincide, and the bright spatial solitons can be formed. 


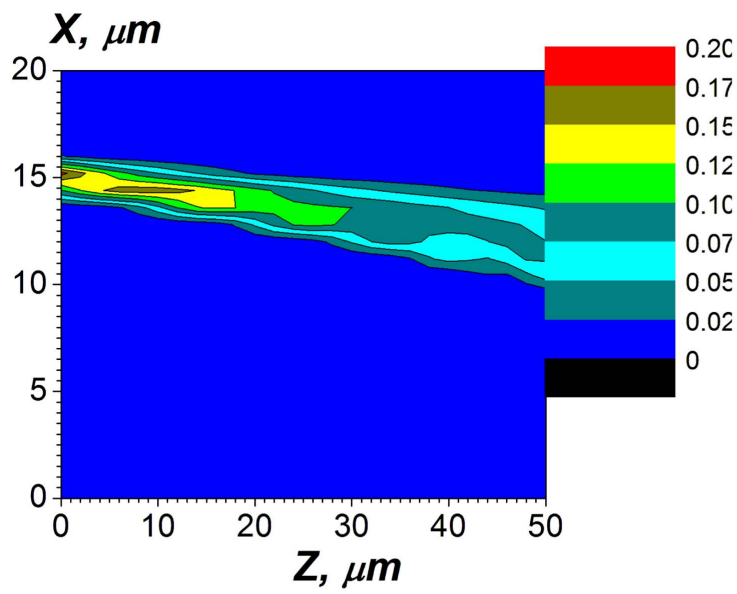

a)

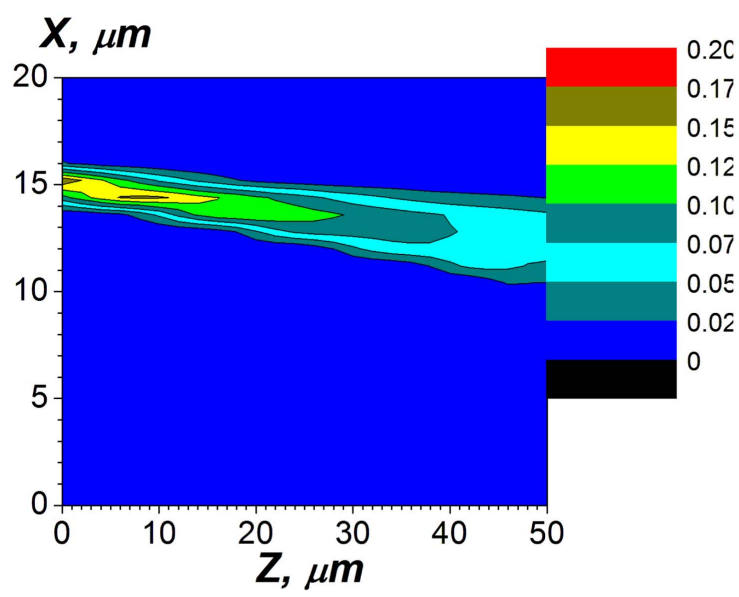

c)

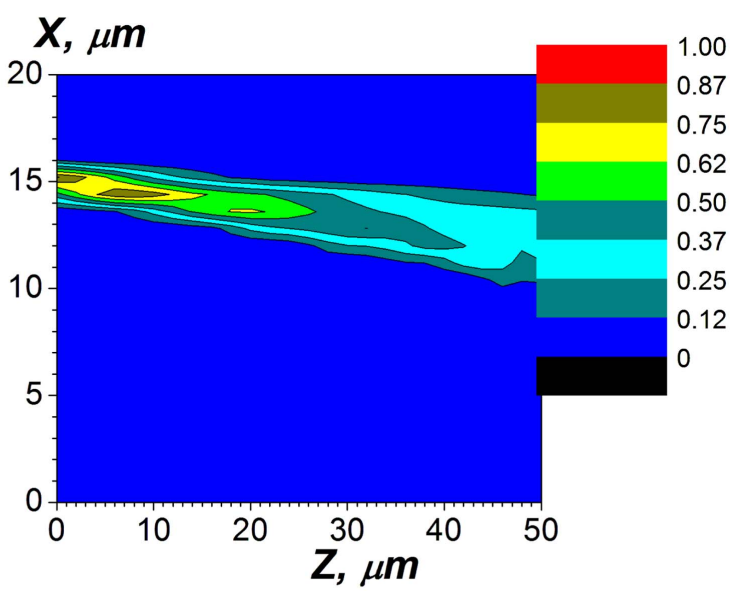

b)

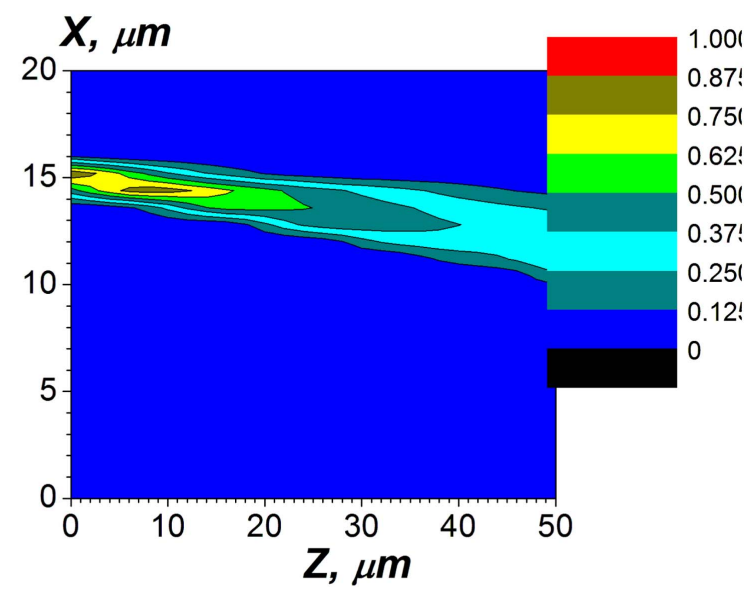

d)

Figure 19. Propagation of nonlinear waves. The circular frequency is $\omega=6 \pi \times 10^{14} \mathrm{~s}^{-1}\left(\lambda_{0}=1 \mu \mathrm{m}\right)$. The nonlinear coefficient for elementary layers 2, 3 is $\alpha=1, \gamma=0.5$. Parts (a), (b) are $|E|^{2}$ and $|H|^{2}$ for the thicknesses of elementary layers $d_{1}=7.5 \mathrm{~nm}$, $d_{2}=d_{3}=3.75 \mathrm{~nm}$. Parts (c), (d) are the same, but the simulations are within the framework of averaged permittivities. For the chosen parameters, the permittivity of the contacting medium is $\varepsilon_{I}=4.203$. The half-width of the incident beam is $x_{0}=1 \mu \mathrm{m}$.

\subsection{The main results and discussion}

The method of averaging can be applied when the thicknesses of elementary layers are small and the nonlinearity is moderate. When the following inequality is valid $d_{i} \leqslant 0.1 \lambda_{i}$ where $d_{i}$ is the thickness of each elementary layer, $\lambda_{i} \equiv \lambda_{0} \cdot \varepsilon_{i}^{-1 / 2}$ is the wavelength of the electromagnetic wave in this medium, the results of simulations are the same both within the direct consideration of the layered medium and within the averaging approach for the hyperbolic medium. In this case it is possible to reduce the nonlinear equation for EM wave propagation to the standard NLSE, where the wave amplitude is slowly varying with respect the longitudinal coordinate, i.e. along the direction of propagation. When the inequalities are valid $0.1 \lambda_{i}$ $\leqslant d_{i} \leqslant 0.25 \lambda_{i}$, there are some quantitative differences within two approaches pointed above but the results are qualitatively similar. At larger thicknesses of elementary layers the differences between two approaches are qualitative and the averaging approach is not valid. The averaging in the metamaterial approximation of the continuous media is based on the formulas (4.10b) and (4.11). These formulas have been derived, accounting for the periodicity of the structure and with the farther application of the approximation of the thin layers (continuous media) in the linear limiting case. For the nonlinear media, the application of the formulas (4.11) in the same form, as that for the linear case, is only phenomenological. This is why it was necessary and important to verify the metamaterial approximation for active nonlinear media (formulas (4.10b) and (4.11)) by means of the comparison between the results of the corresponding modelling with these obtained using more accurate approach without an averaging (based on the equation (4.4)). We would like to emphasise that the positive result of such a comparison is obtained in the present work only for the case of the nonlinear active media with net zero gain (total compensation between gain and losses). A possibility of the metamaterial approach to the hyperbolic nonlinear periodical active media with the nonzero net gain, as to the continuous media is questionable, even providing that the requirements of the metamaterial 


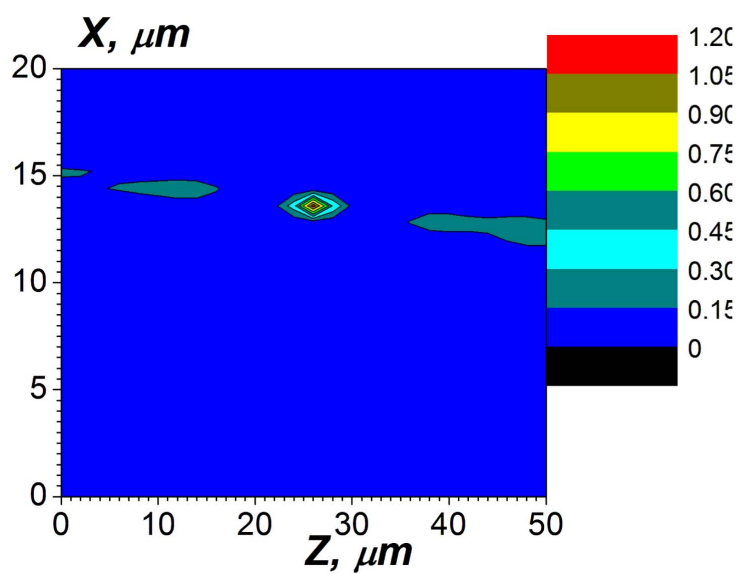

a)

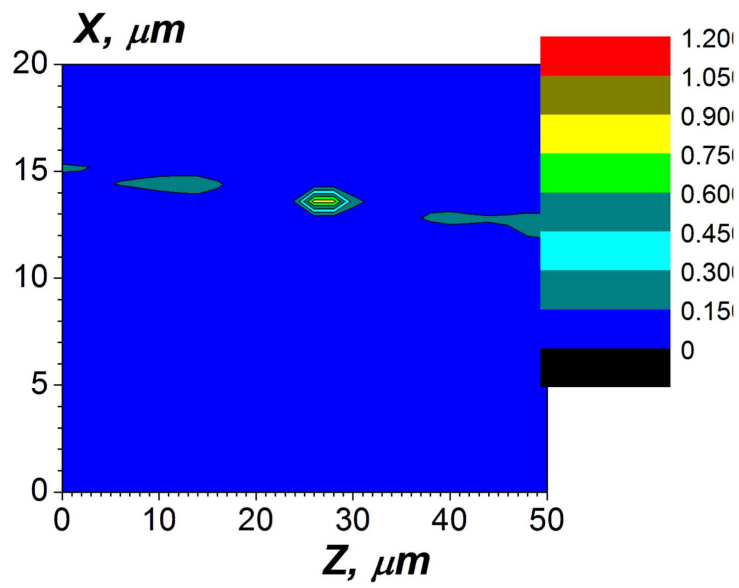

c)

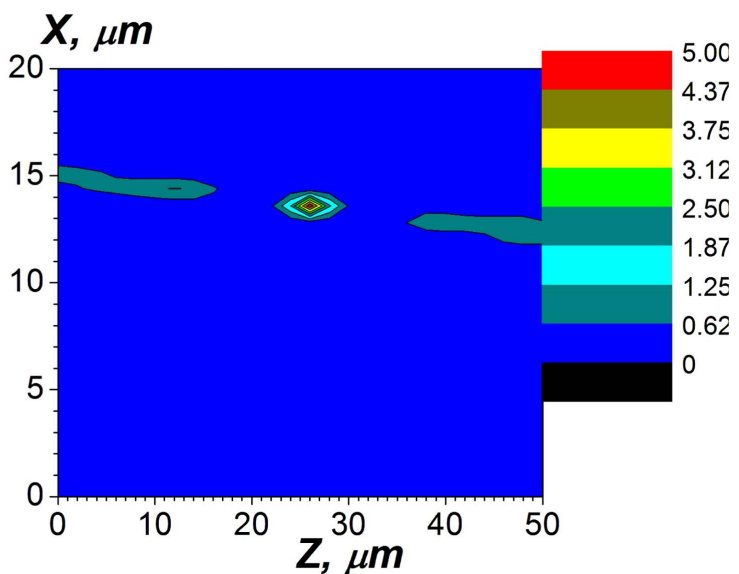

b)

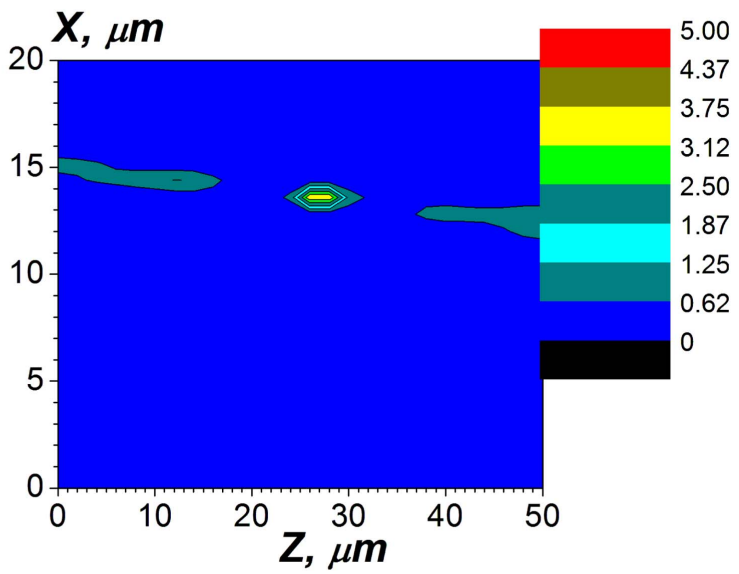

d)

Figure 20. Propagation of nonlinear waves. The circular frequency is $\omega=6 \pi \times 10^{14} \mathrm{~s}^{-1}\left(\lambda_{0}=1 \mu \mathrm{m}\right)$. The nonlinear coefficient for elementary layers 2, 3 is $\alpha=-1, \gamma=0.5$. Parts (a), (b) are $|E|^{2}$ and $|H|^{2}$ for the thicknesses of elementary layers $d_{1}=7.5 \mathrm{~nm}$, $d_{2}=d_{3}=3.75 \mathrm{~nm}$. Parts (c), (d) are the same, but the simulations are within the framework of averaged permittivities. For the chosen parameters, the permittivity of the contacting medium is $\varepsilon_{I}=4.203$. The half-width of the incident beam is $x_{0}=1 \mu \mathrm{m}$.

approximation applicability are satisfied in the linear limiting case. These problems will be a subject of the next paper.

For solving nonlinear problems various difference schemes have been applied. The implicit-explicit method of Peaceman-Rachford does not possess good stability. The method of the summatory approximation needs very small temporal steps and is practically not applicable. It is very interesting, that the method of the operator factorisation, or the method of Douglas-Rachford (known more in the hydrodynamics [67], than in the nonlinear optics), seems the most appropriate.

Nonlinear effects are different for different signs of nonlinearity. In the case of the negative nonlinearity of the layers with positive permittivity the hotspots can be formed within the hyperbolic medium. In the case of the positive nonlinearity of the layers with positive permittivity the nonlinear diffraction of the EM wave beams occurs.

In near-infrared and visible optical range the metallic (or semi-metallic) layers with high conductivity can be used as media with the negative permittivity, whereas the dielectrics with a high values of permittivity can be used as another layers. In the $\mathrm{THz}$ range the narrow forbidden gap semiconductors, like $n$-InSb, can be used as media with the negative permittivity. The metallic layers possess dissipation, even in the metals with high conductivity. Therefore, the dissipation should be compensated, to observe the nonlinear wave phenomena. A mechanism of compensation of dissipation can be a creation of active dielectric layers, for instance, by means of inserting quantum dots with the inversion of energetic levels. In this paper only the case of exact compensation is considered. The dynamics of nonlinear waves under moderate net amplification is of great interest, very non-trivial, in accordance with our preliminary evaluations, and is a subject of future work. The first approach described in the section 4.2 including equation (4.4) is the appropriate one and will be applied for a solution of such a problem(s). In particular, the question of a possibility of stationary regimes as such, while the hotspots are formed in the nonlinear media with a wave amplification is, again, very non-trivial and will be considered elsewhere. 


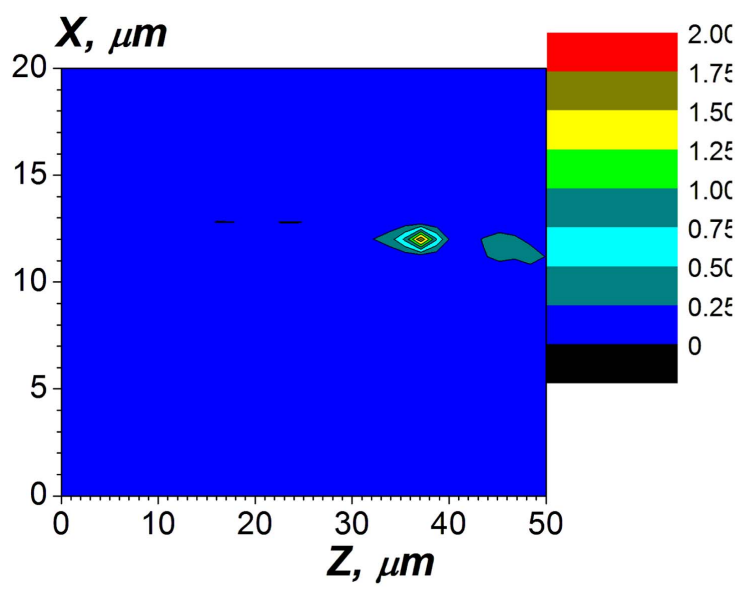

a)

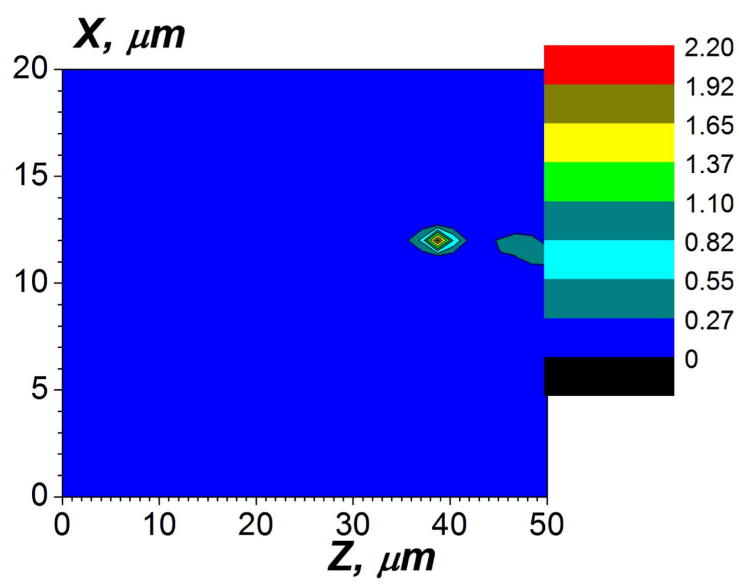

c)

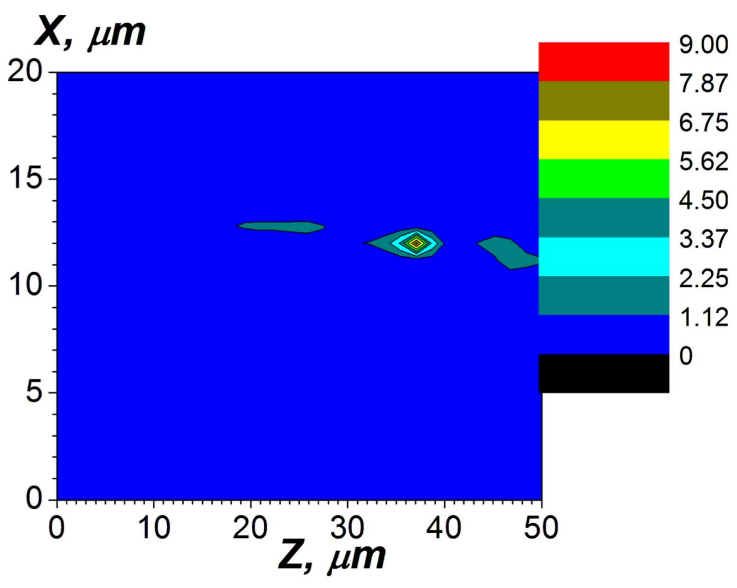

b)

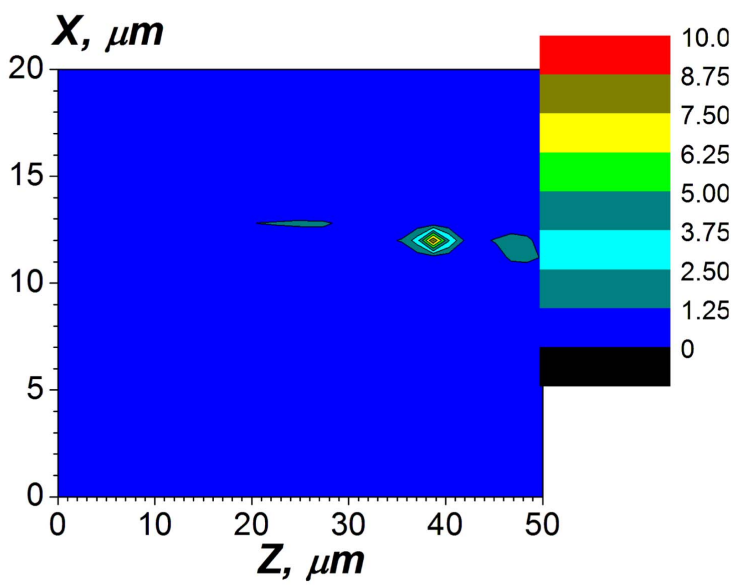

d)

Figure 21. Propagation of nonlinear waves. The circular frequency is $\omega=6 \pi \times 10^{14} \mathrm{~s}^{-1}\left(\lambda_{0}=1 \mu \mathrm{m}\right)$, the incidence angle is $\theta_{i}=30^{\circ}$, the amplitude of the incident beam is $A_{0}=1$. The nonlinear coefficient for elementary layers 2,3 is negative $\alpha=-1, \gamma=0.5$. Parts (a), (b) are $|E|^{2}$ and $|H|^{2}$ for the thicknesses of elementary layers $d_{1}=7.5 \mathrm{~nm}, d_{2}=d_{3}=3.75 \mathrm{~nm}$. Parts (c), (d) are the same, but the simulations are within the framework of averaged permittivities. For the chosen parameters, the permittivity of the contacting medium is $\varepsilon_{I}=4.203$. The half-width of the incident beam is $x_{0}=2 \mu \mathrm{m}$, the centre of the incident beam is $x_{1}=14 \mu \mathrm{m}$.

\section{Spatial solitons in hyperbolic metamaterials}

In this section we investigate the formation and propagation of optical spatial solitons in hyperbolic metamaterials. To model the response of hyperbolic metamaterials we use the homogenisation (or 'effective medium', see also section 4 [71]) theory, with a macroscopic dielectric tensor encompassing at least one negative eigenvalue. We first show that light propagating in the presence of hyperbolic dispersion undergoes negative (anomalous) diffraction. Then we discuss how the solitons features - width and trajectory - depend on the orientation of the optical axis $\hat{n}$, i.e., its angle with the wavevector.

Let us consider a non-magnetic uniaxial medium described by the dielectric permittivity tensor $\epsilon$. Uniaxiality implies the existence of just two independent eigenvalues for $\epsilon$, corresponding respectively to $\epsilon_{\perp}$ (double multiplicity, for electric fields normal to the optical axis) and $\epsilon_{\|}$(single multiplicity, for electric fields parallel to the optical axis). In hyperbolic metamaterials, one of two eigenvalues is negative [72]. The linear propagation of monochromatic light in homogeneous media is fully determined by the dispersion relationship $k_{z}=k_{z}\left(k_{y}\right)$. The isofrequency curves in the plane $k_{z} k_{y}$ undergo a topological transition according to the sign of the permittivity eigenvalues. For elliptic $\left(\epsilon_{\perp}>0, \epsilon_{\|}>0\right)$ and hyperbolic $\left(\epsilon_{\perp} \epsilon_{\|}<0\right)$ media the curves are ellipses and hyperbolae, respectively $[31,72]$. In the hyperbolic case the two possible conditions $\epsilon_{\|}<0$ and $\epsilon_{\|}>0$ define type I and type II materials, respectively.

In uniaxial crystals the elements of the dielectric tensor $\epsilon$ can be written as $\epsilon_{i j}=\epsilon_{\perp} \delta_{i j}+\epsilon_{a} n_{i} n_{j}(i, j=x, y, z)$ (see section 3.2), where $\delta_{i j}$ stands for the Kronecker's delta, $n_{i}$ are the Cartesian components of the optical axis $\hat{n}$, and $\epsilon_{a}=\epsilon_{\|}-\epsilon_{\perp}$ is the optical anisotropy. In order to avoid coupling between ordinary and extraordinary components, we assume exclusively in-plane rotation of $\hat{n}$ in the plane arbitrarily chosen as $y z$. Thus, the knowledge of the angle $\theta$ between the optical axis $\hat{n}$ and the axis $z$ suffices to univocally 
(a)

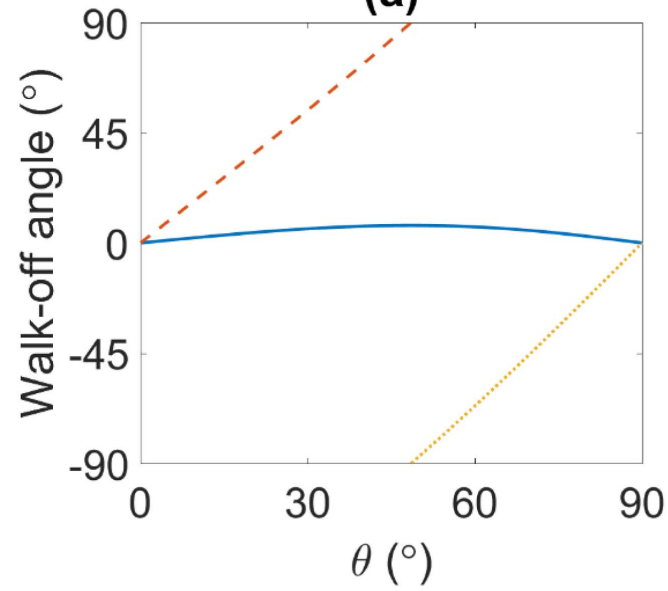

(b)

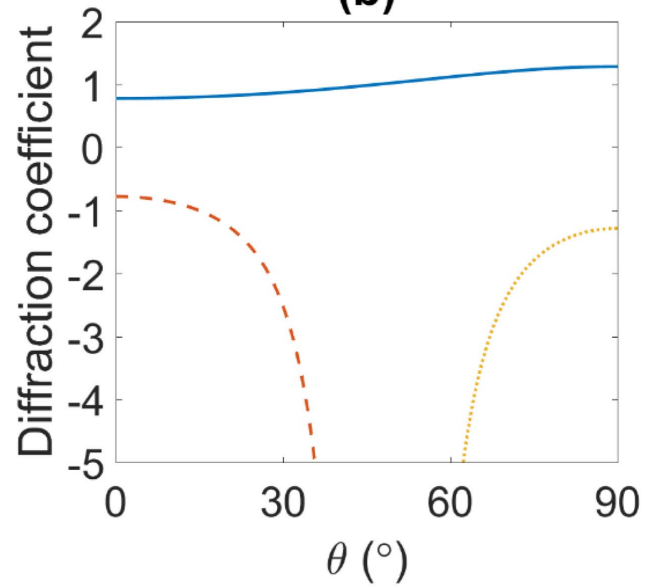

Figure 22. Walk-off angle $\delta$ (a) and diffraction coefficient $D_{y}$ (b) versus the angle $\theta$ between optical axis and wavevector for an elliptic medium with $\epsilon_{a}>0$ (blue solid line), a hyperbolic type I (dashed red line) and a hyperbolic type II (dotted orange line). Here the absolute values of the permittivity eigenvalues are $\left|\epsilon_{\perp}\right| / \epsilon_{0}=2.25$ and $\left|\epsilon_{\|}\right| / \epsilon_{0}=2.89$. The edge of the existence region for homogeneous plane waves is at $48.6^{\circ}$.

determine the optical response. The dielectric tensor $\epsilon$ then reads

$$
\boldsymbol{\epsilon}=\left(\begin{array}{ccc}
\epsilon_{x x} & 0 & 0 \\
0 & \epsilon_{y y} & \epsilon_{y z} \\
0 & \epsilon_{z y} & \epsilon_{z z}
\end{array}\right),
$$

where, in general, all the elements of $\epsilon$ can vary with position. Since we are interested in the extraordinary wave, we can assume that the electric field oscillates in the plane $y z$. For the sake of simplicity, we consider a two-dimensional structure by setting $\partial_{x}=0$ and account for the nonlinear response through a generic nonlinear polarisation $\boldsymbol{P}_{\mathrm{NL}}$. To avoid nonlinearity-induced coupling between the ordinary and the extraordinary waves, we set $\boldsymbol{P}_{\mathrm{NL}} \cdot \hat{x}=0$. If the spatial derivatives of $\epsilon_{i j}$ are neglected, Maxwell's equations can be recast in the form [64, 73]

$$
\begin{gathered}
E_{z}=-\frac{\mathrm{i}}{\omega \epsilon_{z z}} \frac{\partial H_{x}}{\partial y}-\frac{\epsilon_{z y}}{\epsilon_{z z}} E_{y}-\frac{1}{\epsilon_{z z}} P_{\mathrm{NL}, z} \\
\frac{\partial H_{x}}{\partial z}+\frac{\epsilon_{y z}}{\epsilon_{z z}} \frac{\partial H_{x}}{\partial y}=-\mathrm{i} \omega\left(\epsilon_{y y}-\frac{\epsilon_{y z} \epsilon_{z y}}{\epsilon_{z z}}\right) E_{y} \\
-\mathrm{i} \omega\left(P_{\mathrm{NL}, y}+\frac{\epsilon_{y z}}{\epsilon_{z z}} P_{\mathrm{NL}, z}\right), \\
\frac{\partial E_{y}}{\partial z}+\frac{\epsilon_{z y}}{\epsilon_{z z}} \frac{\partial E_{y}}{\partial y}=-\mathrm{i} \omega \mu_{0} H_{x}-\frac{\mathrm{i}}{\omega \epsilon_{z z}} \frac{\partial^{2} H_{x}}{\partial y^{2}}-\frac{1}{\epsilon_{z z}} \frac{\partial P_{\mathrm{NL}, z}}{\partial y} .
\end{gathered}
$$

Under the assumptions above, equations (5.1)-(5.3) govern light propagation in the presence of any type of nonlinearity, both in the paraxial and non-paraxial regimes, regardless of the nature of the elements $\epsilon_{i j}$ (which can be complex or real, either positive or negative).

Hereafter we exclude the presence of gain or losses by considering a Hermitian dielectric tensor (i.e., $\epsilon_{i j}=\epsilon_{i j}^{*}$ ). A general result on light propagation in the linear regime $\left(\boldsymbol{P}_{\mathrm{NL}}=0\right)$ can be derived from equations (5.1)-(5.3). Let us consider a beam propagating in the anisotropic medium with wavevector parallel to the axis $z$. The evolution operator on the lhs of equations (5.2) and (5.3) suggests that light propagation occurs as in isotropic materials, but in the frame $y^{\prime}=y-\frac{\epsilon_{y z}}{\epsilon_{z z}} z, x^{\prime}=x$ and $z^{\prime}=z$, analogously to a swimmer in a flowing river [64]. The latter transverse velocity is called walk-off and is well known in optics as linear birefringence [74]. Walk-off manifests itself as a non-vanishing angle $\tan \delta=\frac{\epsilon_{y z}}{\epsilon_{z z}}$ between the wavevector and the Poynting vector $S$, i.e., an energy flow (i.e., rays) non-orthogonal to the wavefronts, at variance with isotropic materials. We stress that, according to the model above, the walk-off angle does not depend on the spatial spectrum (or transverse size) of the beam, a result hardly achievable using plane wave expansions [75]. The calculated walk-off angle versus the optical axis angle $\theta$ is plotted in figure 22(a). In elliptic media with a small positive anisotropy, the walk-off is maximised for $\theta \approx 45^{\circ}$ [76]. Conversely, in hyperbolic media the walk-off increases monotonically with the angle $\theta$, reaching an absolute value of $90^{\circ}$ at the edge of the existence region, when surface waves are excited at the input interface of a hyperbolic sample [77].

We just ascertained the beam trajectory by investigating the role of walk-off. Another important figure is the beam width versus propagation. In order to determine the beam width, we need to rewrite equations (5.1)-(5.3) as a single Helmholtz equation. When $\frac{\epsilon_{y z}}{\epsilon_{z z}}$ i.e., the walk-off angle-, undergoes small variations in the transverse direction $(y)$ and adiabatic changes in the longitudinal direction $(z)$, we can rewrite equations (5.1)-(5.3) in the moving frame $x^{\prime} y^{\prime} z^{\prime}$ defined above. Then, optical propagation can be fully described by a magnetic field satisfying $[64,73]$

$$
\begin{aligned}
& \frac{\partial^{2} H_{x}}{\partial z^{\prime 2}}+D_{y} \frac{\partial^{2} H_{x}}{\partial y^{\prime 2}}+k_{0}^{2} n_{e}^{2} H_{x} \\
& =-\mathrm{i} \omega \frac{\partial\left(P_{\mathrm{NL}, y}+\tan \delta P_{\mathrm{NL}, z}\right)}{\partial z^{\prime}}+\frac{\mathrm{i} \omega n_{e}^{2} \epsilon_{0}}{\epsilon_{z z}} \frac{\partial P_{\mathrm{NL}, y}}{\partial y^{\prime}},
\end{aligned}
$$


where $n_{e}^{2}=\frac{\epsilon_{y y}}{\epsilon_{0}}-\frac{\epsilon_{y z}^{2}}{\epsilon_{0} \epsilon_{z z}}$ is the refractive index for extraordinary plane waves propagating along $z$, and $D_{y}=\frac{\epsilon_{0} n_{e}^{2}}{\epsilon_{z z}}$ is the diffraction coefficient. Since the existence of homogeneous propagating waves requires a real refractive index $n_{e}$, non-attenuated plane waves always exist in elliptic materials, whereas they exist in hyperbolic media only for some angles $\theta$, in agreement with the hyperbolic isofrequency curve [72]. The diffraction coefficient can also be expressed as

$$
D_{y}=\frac{\epsilon_{\perp} \epsilon_{\|}}{\epsilon_{z z}^{2}} .
$$

In hyperbolic media it is $\epsilon_{\perp} \epsilon_{\|}<0$, thus equation (5.5) predicts negative (or anomalous) diffraction [73]. In figure 22(b) the diffraction coefficient $D_{y}$ is plotted versus the optical axis orientation given by $\theta$. While in the elliptic case non-attenuated light propagation is always allowed and the diffraction coefficient slightly differs from unity for small anisotropies [76], the topological transition in the dispersion strongly affects the diffraction: in fact, a plane wave expansion provides $D_{y}=-\left.k_{0} n_{0} \frac{\partial^{2} k_{z}}{\partial k_{y}^{2}}\right|_{k_{y}=0}$ [78], corresponding to the concavity of the spatial dispersion $k_{z}\left(k_{y}\right)$. Figure 22(b) plots the values derived from equation (5.5). $D_{y}$ is always real and negative, with a singularity at the edge of the existence region.

The evolution of the beam width is determined by $D_{y}$. To see that, let us consider light propagation in a linear and homogeneous dielectric. Equation (5.4) then turns into a standard paraxial Helmholtz equation with respect to the normalised transverse coordinate $\eta=\frac{y^{\prime}}{\sqrt{ } D_{y}}$. For example, for a fundamental Gaussian beam of waist $w$ the Rayleigh length $L$ is $L=\frac{\pi n_{e}^{2} w^{2}}{D_{y} \lambda}$ [76]. The physical outcomes of $D_{y}<0$ can be grasped by writing equation (5.4) in the paraxial limit [73]

$$
\begin{aligned}
\mathrm{i} \frac{\partial A}{\partial z^{\prime}}= & -\frac{D_{y}}{2 k_{0} n_{0}} \frac{\partial^{2} A}{\partial y^{\prime 2}}-\frac{k_{0}}{2 n_{0}}\left(n_{e}^{2}-n_{0}^{2}\right) A-\frac{\mathrm{i} \omega}{2 k_{0} n_{0}} \\
& \times \frac{\partial\left(P_{\mathrm{NL}, y}+\tan \delta P_{\mathrm{NL}, z}\right)}{\partial z^{\prime}}+\frac{\mathrm{i} \omega n_{e}^{2} \epsilon_{0}}{2 k_{0} n_{0} \epsilon_{z z}} \frac{\partial P_{\mathrm{NL}, y}}{\partial y^{\prime}},
\end{aligned}
$$

where the slowly varying envelope $A$ is defined through $H_{x}=A\left(y^{\prime}, z^{\prime}\right) \mathrm{e}^{\mathrm{i} k_{0} n_{0} z^{\prime}}$. Equation (5.6) is a Schrödinger equation with effective time $z$ for a particle of mass $\frac{k_{0} n_{0}}{D_{y}}$ subject to a photonic potential $-k_{0}\left(n_{e}^{2}-n_{0}^{2}\right) /\left(2 n_{0}\right)$. Thus, in the presence of hyperbolic dispersions, a wavepacket behaves like a particle of negative mass [73]. The latter analogy paves the way to the prediction of interesting phenomena. First, optical propagation in homogeneous hyperbolic media can be modelled as a time-inverted propagation. As a direct consequence, a hyperbolic slab of appropriate length can compensate the diffraction occurred in an isotropic slab, thus permitting the recovery of the initial field distribution [73]. Second, if compared to elliptic dispersion, light beams react in the opposite way to gradients in the refractive index, with photons now being attracted (pulled) towards regions with lower refractive index [73].
Such exotic response to an index gradient has important consequences on the design and realisation of dielectric waveguides in hyperbolic metamaterials, with optical confinement achievable when the refractive index is lower in the core than in the periphery of the waveguide (cladding and substrate in a planar waveguide).

We are now able to discuss the main properties of optical spatial solitons in hyperbolic media. For the sake of simplicity, we assume a medium with a local Kerr-like nonlinear response and a homogeneous linear permittivity. Thus, the nonlinear polarisation can be modelled as a refractive index change dependent on the local intensity as [67]

$$
n_{e}^{2}-n_{0}^{2}=n_{2}|A|^{2}
$$

When equation (5.7) is valid, equation (5.6) becomes a standard NLSE, thus supporting solitons with a hyperbolic secant profile [67]. The detailed analysis of soliton properties for other types of nonlinearities can be carried out starting from equation (5.6) and considering the exact nonlinear dielectric tensor.

To address the role of negative diffraction, we first consider soliton propagation in the absence of walk-off. The main difference with respect to isotropic materials is the link between dark/bright solitons, and the sign of the Kerr coefficient $n_{2}[79,80]$. In full analogy with the case of temporal solitons, self-focusing (defocusing) corresponds to a negative (positive) Kerr coefficient [67, 81, 82]; hence, dark and bright solitons are supported by hyperbolic metamaterials when $n_{2}>0$ and $n_{2}<0$, respectively (see also equation (4.23b) and relate discussion in section 4.5) [73]. Such result can be intuitively understood from the properties of a linear waveguide in hyperbolic materials, considering that a (first-order) spatial soliton is the fundamental mode of the guide written by the soliton itself [83]. Examples of light propagation in a type I material with $\theta=0$ computed via a BPM code based on the Crank-Nicolson algorithm and operator splitting are plotted in figures 23(a)-(c). As predicted, self-focusing and the formation of bright solitons can be observed when the Kerr coefficient is negative (panel (a)). Conversely, beams undergo self-defocusing (i.e., diffractive spreading larger than in the linear regime) when the Kerr nonlinearity is positive. Moreover, both the nonlinear effects are enhanced when the input power is increased.

We now consider the formation of bright spatial solitons as the angle $\theta$ varies and the normalised excitation $n_{2} P$ is clamped to a negative value. The most apparent effect is a change in beam trajectory owing to the different walk-off angle $\delta$, see figures 23(d)-(i) [84]; in type I (II) materials, the walk-off angle spans from 0 to 90 ( -90 to 0 ) degrees (see figure 22(a)). For different $\theta$, the beam profile changes as well owing to the different amount of diffraction, even if the normalised power $n_{2} P$ is kept constant. Accordingly, the size of the self-focused wavepacket gets larger as the angle $\theta$ approaches the edge of the existence region, i.e., when diffraction diverges.

To conclude this section, let us mention a few forthcoming developments and generalisations of this work. The first is the solution of equation (5.4) employing the nonlinear 


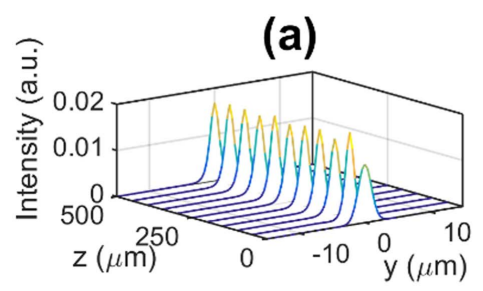

(d)

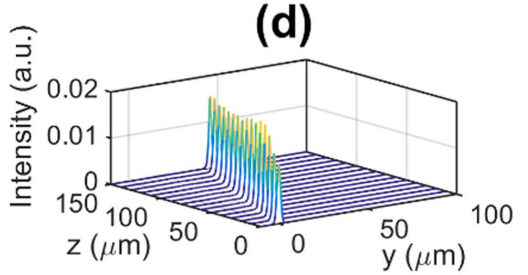

(g)

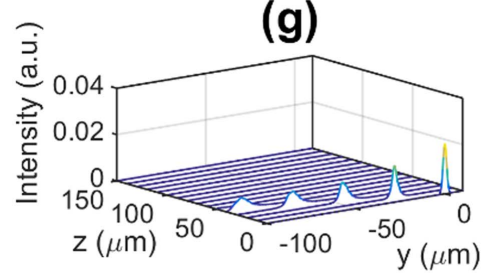

(b)

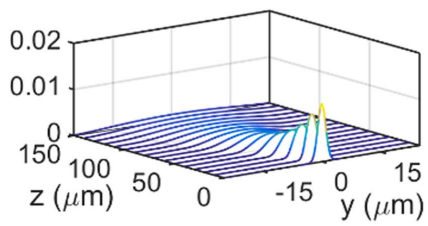

(e)

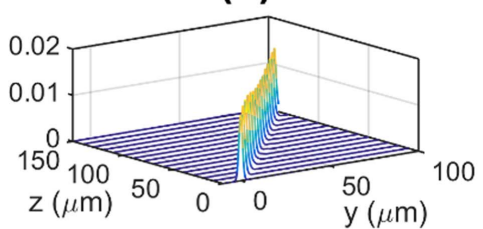

(h)

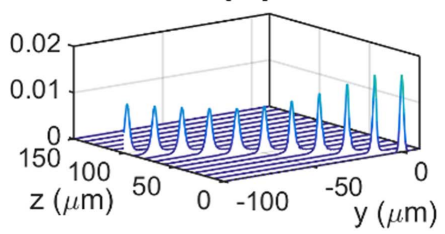

(c)

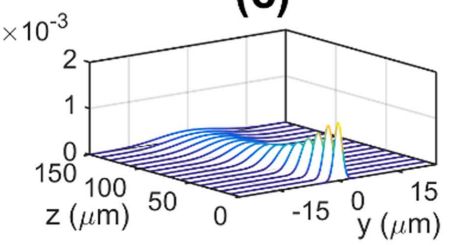

(f)

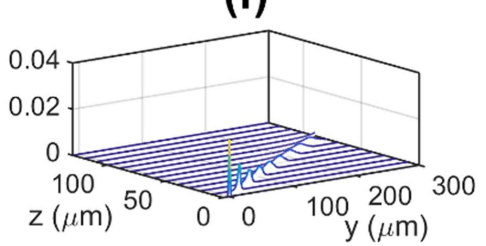

(i)

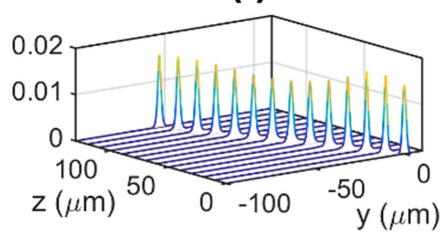

Figure 23. Nonlinear light propagation when the input is a Gaussian beam of waist $2 \mu \mathrm{m}$, planar phase front and wavelength $\lambda=1064 \mathrm{~nm}$. (a)-(c) Intensity evolution for $\theta=0$ when (a) $\frac{n_{2} P}{Z^{2}}=-1 \times 10^{-11} \mathrm{~m}^{2} \mathrm{~V}^{-2} \mathrm{~W}\left(\mathrm{Z}\right.$ is the medium impedance), (b) $\frac{n_{2} P}{Z^{2}}=1 \times 10^{-11} \mathrm{~m}^{2} \mathrm{~V}^{-2} \mathrm{~W}$, and (c) $\frac{n_{2} P}{Z^{2}}=5 \times 10^{-12} \mathrm{~m}^{2} \mathrm{~V}^{-2} \mathrm{~W}$; the considered medium is a type I hyperbolic media. (d)-(f) Intensity evolution in a type I hyperbolic media for $\theta=10^{\circ}$ (d), $\theta=20^{\circ}$ (e) and $\theta=40^{\circ}$ (f) for $\frac{n_{2} P}{Z^{2}}=-1 \times 10^{-11} \mathrm{~m}^{2} \mathrm{~V}^{-2} \mathrm{~W}$. (g)-(i) Intensity evolution in a type II hyperbolic media for $\theta=60^{\circ}$ (d), $\theta=70^{\circ}$ (e) and $\theta=80^{\circ}$ (f) for $\frac{n_{2} P}{Z^{2}}=-1 \times 10^{-11} \mathrm{~m}^{2} \mathrm{~V}^{-2} \mathrm{~W}$. Absolute values of the permittivity eigenvalues are $\left|\epsilon_{\perp}\right| / \epsilon_{0}=2.2614$ and $\frac{\left|\epsilon_{\|}\right|}{\epsilon_{0}}=2.8744$, respectively.

tensor permittivity of an actual medium, either of second or third order or both [67]. In fact, since equation (5.4) is a complete vectorial model for the propagation of the electromagnetic field, it allows one to address, for example, the role of longitudinal fields when self-trapping reaches scales comparable with the wavelength [85]. Another important point is the accurate assessment of the homogenisation theory by solving the complete Maxwell's equations in a real hyperbolic metamaterial [86, 87]. In fact, our simplified model does not consider the role played by the unavoidable losses $[78,88,89]$ and the inherent spatial nonlocality of the response $[90,91]$.

\section{Rogue waves in metamaterial waveguides}

\subsection{Introduction and background}

The theme of this section is optical rogue waves. However, in this case, the waves will be subject to a metamaterials environment and also a magnetooptic influence. It is clear that optical rogue waves have been written about very vigorously in the literature. Historically and currently it is the dispersive type of NLSE that has been used and if rogue waves were not the main interest then it would be temporal solitons that emerge from this type of equation. The question of whether spatial solitons could be used will be discussed later on.
Metamaterials and optical rogue waves have both been making headlines in recent years and they are separately large areas of research to study. Here a brief background of the almost inevitable linkage of these two fascinating subjects is considered with important new possibilities being discussed. Initially we consider the background then show interesting new results combining the two areas and go on to discuss the way forward for the future.

A rogue is broadly considered to be something that does not follow the generally accepted path. In the context of what is being discussed here, in an analogous fashion to hydrodynamic rogue waves, we consider optical phenomena that do not follow the traditionally accepted path with peaks of intensity that appear to spring out from an almost flat background (albeit with a small perturbation) only to decay away again with equal rapidity. In the section below one scenario considered is the famous case of a single peak appearing and disappearing, known as a Peregrine soliton, and in a subsequent scenario a multi-peak solution is discussed.

It is worth pointing out that such rogues have been observed but are a comparatively rare event in nature. The reason for this is that it is a relatively low probability that any natural physical system has precisely the boundary condition that leads to rogues. When considering hydrodynamic rogue waves it was until relatively recently difficult to make appropriate measurements at sea. This lack of natural evidence led for a long time to a disbelief about rogue wave 
events. However, interestingly, it is possible to generate them in laboratory experiments. Indeed, convenient fibre-based experiments taking advantage of high-speed telecommunication-grade components have recently confirmed the existence of extreme wave phenomena that can occur both in optical systems and in hydrodynamics [92-97]. Analogies between hydrodynamics and optics have been known since the 1960-1970s thanks to two main findings: (1) the derivation of the soliton solution of the NLSE in the form of secanthyperbolic shaped (temporal or spatial) profile, and (2) the studies of the Benjamin-Feir/Bespalov-Talanov (or modulation) instability [98-104].

Wave dynamics in weakly nonlinear dispersive media, such as in optical Kerr media or on the surface of deep water, can indeed be described by the NLSE. However, it was recently shown that this correspondence applies even in the limit of extreme nonlinear wave localisation described by the common mathematical model $[96,97]$. In particular, note that the NLSE admits general breather solutions on a finite background, i.e. pulsating envelopes that well mimic the dynamics of rogue waves that may appear from nowhere and disappear without leaving a trace. As a consequence, we can first address the issue of rogue waves in (almost-) conservative systems in terms of NLSE breathers whose entire space-time evolution is analytically described [105]. Their pulsating and localisation properties make such mathematical solutions the simplest nonlinear prototypes of the famous hydrodynamic rogue waves [106], in particular the doubly localised (in space and time) breather solutions (i.e. Peregrine soliton and higher orders) [107]. These pulsating solutions also include solutions that are either periodic in space and localised in time or periodic in time and localised in space which are are refered to as Kuznetsov-Ma breathers and Akhmediev breathers respectively. Taking the period of both of these latter solutions to infinity gives rise to the Peregrine solution.

If we now recall that breather solutions describe localised carrier perturbations with a strong amplification, they provide support to the nonlinear stage of the universal MI phenomenon [108]. We distinguish two MI regimes: on the one hand, the noise-driven MI that refers to the amplification of initial noise superposed to the plane wave that leads to spontaneous pattern formation from stochastic fluctuations; on the other hand, the coherent seeded MI (or coherent driving of MI) that refers to the preferential amplification of a specific perturbation (i.e., leading to a particular breather solution) relative to any broadband noise. In either case, the wave dynamics can be interpreted in terms of breathers and competitive interactions. Most importantly, the coherent seeded MI can be used to efficiently stabilise and manipulate the output wave, thus allowing it to generate and quantitatively measure NLSE breather properties in optical fibres.

It is also important to say that breather dynamics appear even with initial conditions that do not fulfil the mathematical ideal and optical studies have strongly contributed to that end. In fact it can be said any kind of disturbance of the ideal NLSE propagation induces a deviation from the expected theoretical solutions (specific to each breather on a finite background), but most of the features related to their pulsating dynamics remain clearly observable. Breathers on a finite background may be considered as 'robust solutions' (but unstable solutions from the mathematical point of view) [109], in the sense that they can be excited or propagated even with non-ideal conditions, and the main features of a localised high amplitude event (i.e., rogue wave) still occur. In general, fibre characteristics are chosen in accordance with both spectral bandwidth and peak power of the wave evolving into the fibre in order to avoid the potential impact of higher-order dispersive or nonlinear effects [110]. Nevertheless, besides fibre losses, higher order effects linked to pulse propagation such as third-order dispersion, self-steepening, and the Raman effect can be considered, even theoretically by extending the NLSE [109]. Exact rogue wave solutions were even found in such complex equations that are integrable in special cases, such as the Sasa-Satsuma or the Hirota equations [111-114].

From a metamaterials perspective the key work that is often considered to be the starting point is that of Veselago in 1967 [115]. However it was not until the 1990s with improved computing power and manufacturing techniques that these materials could be studied in great depth and in 2000 John Pendry [116] extended Veselago's work to creating the perfect lens. Since then there has been an explosion of papers in the metamaterials field. Metamaterials offer the potential to control a range of electromagnetic behaviour in ways that cannot be achieved with materials found in nature and the ability to create purpose built materials to fulfil new and novel functionality where these artificial structures, that exert influence over electromagnetic waves at the sub-wavelength level, are extensively being studied and designed. Here we contribute to this expansion.

The study of rogue waves in metamaterials is currently at an early stage, only a few recent numerical studies based on a transmission line model (with NLSE reduction) or a cubicquintic NLSE can be found. In particular, it was shown there is the possibility of producing extreme waveform events, with strong similarities to NLSE breather waves (Peregrine, but also Akhmediev or Kuznetsov-Ma breathers) [117-119]. In the following, we bring together both of these subjects by investigating the propagation behaviour of wave excitation corresponding to rogue breather solutions in transparent metamaterials with the addition of magnetooptic properties; wherein higher-order dispersive or nonlinear effects are included. Hence the fascinating idea of rogue wave emergence in the form of Peregrine solitons (and near-Peregrines) within a nonlinear metamaterial environment and where potentially magnetooptic control could be exerted is researched as a new pathway.

\subsection{Simulations}

The world is becoming very interested in hyperbolic metamaterials and breather type solutions however for this type of metamaterial appropriate extensions to the dispersion type NLSE are not yet available. As the best way to investigate rogue waves is based on the dispersion type of NLSE the use of double negative media is the way forward where 
appropriate extensions to the NLSE have already been developed and could thus be readily adapted for the purpose of studying rogue waves. The question of whether the work can be moved into hyperbolic metamaterials is a question for another time.

In this section with the dispersion based NLSE we are looking at solutions of the type that have a flat background with a small perturbation. We assume the material is isotropic and it has a negative permittivity and negative permeability thus the form of the metamaterials considered here is transparently double-negative [120] and our aim is to give some new physical insights on how the metamaterial properties can affect rogue waves in the form of NLSE breathers. More particularly, we reveal that their dynamical behaviour can be influenced and controlled, by metamaterial effects, namely the self-steepening, and magnetooptic effects. Here we restrict our analysis to temporal forms of rogue solutions, however, it is worth mentioning that both spatial and temporal waveform solutions can be considered in metamaterials, in a similar way to the standard soliton solution [120, 121].

The general approach to the NLSE is to assume that the components (both electric and magnetic fields) of an electromagnetic wave propagating in a metamaterial can be factorised such that there is an amplitude that is slowly varying along the propagation direction, a linear modal field contribution and a fast plane wave variation that introduces a propagation wave number. Here a modified approach is adopted such that an appropriate extension to the NLSE [120] is utilised that takes into account the metamaterial properties with self-steepening and magnetooptic effects.

Although both electric and magnetic nonlinearities could be included for a given metamaterial, it has been shown [121] that the effective nonlinearity can be combined into a single nonlinear coefficient. Here however it is assumed that the nonlinear behaviour discussed originates from an isotropic Kerr dielectric. A typical waveguide structure that is used here is shown in figure 24 . It is a planar structure with a metamaterial core that has boundaries in $x$ and $y$ directions and propagation is along the $z$-axis.

The structure is set so that it is weakly guiding as discussed in section 2 of [120]. When part of the structure (as shown in figure 24), in the form of the substrate, is replaced with a magnetooptic material, it is possible to control solitons with the application of a magnetic field. Here the magnetic field is applied in what is known as a Voigt configuration. This has an externally applied magnetic field in the plane of the guide perpendicular to the direction of optical propagation, which here can be applied along either the positive or negative $x$-direction.

The form of the extended NLSE is given below in equation (6.1) and is as discussed in equation (2.22) of [120]. It is noted that there is also the capability of adding higher order and Raman scattering effects however in the simulations discussed below these parameters are not invoked, but will be utilised in future work. This form of the NLSE does not yet contain the magnetooptic parameters, which will be

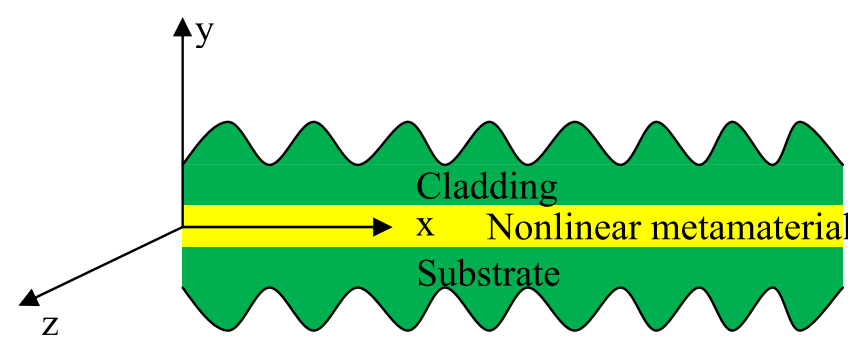

Figure 24. A diagram of a possible waveguide system with negative phase nonlinear metamaterial.

discussed further below.

$$
\begin{aligned}
& \mathrm{i} \frac{\partial \psi}{\partial Z}-\frac{1}{2} \operatorname{sgn}\left(\beta_{2}\right) \frac{\partial^{2} \psi}{\partial t^{2}}-\mathrm{i} \delta_{3} \frac{\partial^{3} \psi}{\partial t^{3}}+\operatorname{sgn}\left(\bar{\chi}^{(3)}\right) \\
& \quad \times\left(|\psi|^{2} \psi+\mathrm{i} S \frac{\partial}{\partial \mathrm{t}}\left(|\psi|^{2} \psi\right)-\tau_{R} \psi \frac{\partial}{\partial \mathrm{t}}\left(|\psi|^{2}\right)\right)=0,
\end{aligned}
$$

where $\beta_{2}$ is the group velocity dispersion, $S$ is the self-steepening coefficient which is discussed below. $\delta_{3}=\beta_{3} /\left(6\left|\beta_{2}\right| t_{0}\right), \beta_{3}$ is the third order dispersion, $t_{0}$ is interpreted as pulse width and $\tau_{R}$ is the Raman coefficient. $\bar{\chi}^{(3)}$ is the third-order nonlinear Kerr coefficient in which the assumption of a weakly guiding system has been embedded. In the simulations presented later in this section the parameters that are considered are self-steepening and the use of a range of magnetooptic parameters that are used to adjust the effect of self-steepening in the metamaterial. It should be noted that the self-steepening coefficient $S$ in equation (6.1) involves very specific properties of the metamaterials which has been pointed out by previous authors $[12,122]$ however here the self-steepening term is that laid out in equation (2.21) of [120] which is arrived at via a different approach and the result is less restrictive than other approximations.

$$
S=\frac{1}{\omega_{0}}\left[2+\frac{\left|v_{p}\right|}{v_{g}}+\left(\frac{\omega}{\mu} \frac{\partial \mu}{\partial \omega}\right)_{\omega_{0}}\right],
$$

where $\omega$ is angular frequency, $\omega_{0}$ is the operational frequency, $v_{p}$ is phase velocity, $v_{g}$ is group velocity and $\mu$ is the permeability and:

$$
\frac{\left|v_{p}\right|}{v_{g}}=-\frac{\beta_{1} \omega_{0}}{\beta_{0}},
$$

where $\beta_{0}$ is the wave number, $\beta_{1}=\left.\frac{\partial k}{\partial \omega}\right|_{\omega_{0}}=\frac{1}{v_{g}}$ it is further noted that the form of the NLSE given here allows for the nonlinear coefficient to be dispersive which if included would give a form for the self-steepening coefficient:

$$
S=\frac{1}{\omega_{0} t_{0}}\left[2-\frac{\beta_{1} \omega_{0}}{\beta_{0}}+\left(\frac{\omega}{\mu} \frac{\partial \mu}{\partial \omega}\right)_{\omega_{0}}+\left(\frac{\omega}{\bar{\chi}^{(3)}} \frac{\partial \bar{\chi}^{(3)}}{\partial \omega}\right)_{\omega_{0}}\right] .
$$

The approach here however is to consider a non-dispersive scenario for $\chi^{(3)}$ thus $\frac{\partial \bar{\chi}^{(3)}}{\partial \omega}=0$. The dispersion of $\chi^{(3)}$ is to be the subject of future work.

The important issue of whether the self-steepening coefficient is affected by metamaterials solutions is discussed in [120] (see figure 3 of [120]) and it is clearly demonstrated that this is the case for a judicious selection of $S$. (In these early stage results similar values to those used in previous 


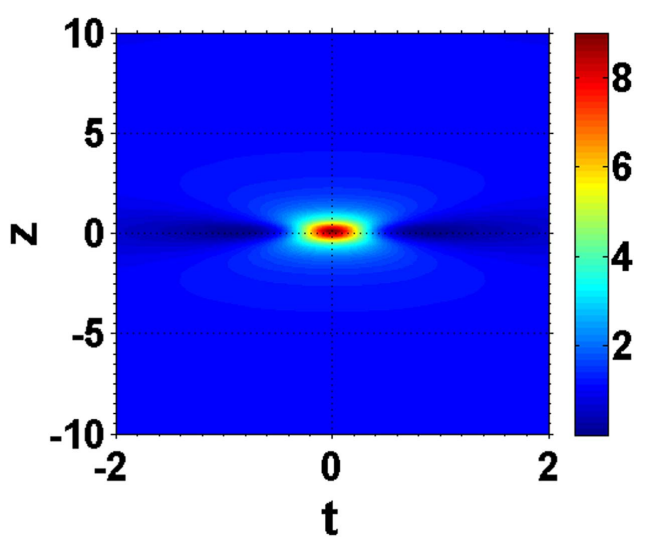

Figure 25. This numerical simulation uses the exact solution of the Peregrine soliton as a starting condition at normalised distance $z=-10$ (at this point none of the extensions to the NLSE are being used). The Crank-Nicolson technique is then used to propagate along the $z$-direction. It can be seen that the result of this process is a single peak at position $z=0$ that subsequently decays in line with the analytical result. It is noted that, although the important perturbation in the initial condition is included, at $z=-10$ the variation on the value of $|\psi|^{2}$ is very close to 1 thus the plot appears almost flat at this point. The dark areas to either side of the main peak centred about $z=0$ are due to the normalised value of $|\psi|^{2}$ dropping below 1 as would be expected.

temporal solitons simulations are adopted to allow comparisons of the different starting conditions used-see below.) The use of typical drude models is adopted that are of the form:

$$
\begin{aligned}
& \varepsilon(\bar{\omega})=\varepsilon_{D}-\frac{1}{\bar{\omega}^{2}}, \\
& \mu(\bar{\omega})=1-\frac{\omega_{m}^{2}}{\omega_{e}^{2}} \frac{1}{\bar{\omega}^{2}},
\end{aligned}
$$

where $\omega_{e}$ and $\omega_{m}$ are plasma frequencies associated with the permittivity and permeability respectively and the dimensionless frequency $\bar{\omega}=\omega / \omega_{e}$. For a specific application $\bar{\omega}=\omega_{0}$, the operational frequency. It is then convenient to write (with a dispersionless $\chi^{(3)}$ ):

$$
\bar{S}=\left(\frac{1}{\varpi}\right) \frac{1}{\omega_{0} T_{0}}\left[2+\frac{\beta_{1} \bar{\omega}}{\left|\bar{\beta}_{0}\right|}+\left(\frac{\bar{\omega}}{\mu} \frac{\partial \mu}{\partial \varpi}\right)\right] .
$$

If the addition of the magnetooptic parameters are now considered it can be shown that:

$$
\begin{gathered}
\mathrm{i} \frac{\partial \psi}{\partial Z}-\operatorname{sgn} \frac{1}{2}\left(\beta_{2}\right) \frac{\partial^{2} \psi}{\partial t^{2}}+\operatorname{sgn}\left(\bar{\chi}^{(3)}\right)|\psi|^{2} \psi+\nu \psi=R, \\
R=-\operatorname{isgn}\left(\bar{\chi}^{(3)}\right)^{2} S \frac{\partial}{\partial t}\left(|\psi|^{2} \psi\right)-\mathrm{i} \delta_{3} \frac{\partial^{3} \psi}{\partial t^{3}}+\tau_{R} \psi \frac{\partial}{\partial t}\left(|\psi|^{2}\right),
\end{gathered}
$$

where here the form of $\nu$ is selected similarly to equation (3.6) of [120] which is:

$$
\nu=\frac{\nu_{\max }}{2}\left(1-\left(\frac{\tanh \left(t-t_{\nu}+\left(\frac{Z}{v_{g}}\right)-2 \Delta t_{v}\right)}{\Delta t_{v}}\right)\right) .
$$

The magnetooptic parameters that are set in the simulations are thus: $t_{\nu}$ the delay of the magnetisation after excitation by the electromagnetic input, $\Delta t_{\nu}$ which is the normalised time over which the magnetisation takes place, $v_{g}$ the group velocity and $\nu_{\max }$, the maximum value of magnetisation which is defined in equation (3.4) in [120] as:

$$
\nu_{\max }=\frac{\omega}{c} L_{D} n_{m}^{2} Q_{\mathrm{sat}},
$$

where $L_{D}$ is the dispersion length, $n_{m}$ is the refractive index of the magnetooptic material and $Q$ is the magnetooptic parameter that is usually taken to define the strength of its influence $Q_{\text {sat }}$ represents the saturation of this parameter. The values selected for $\nu_{\max }$ thus reflect the parameters in equation (6.11). For ordinary magnetooptic materials $Q$ is typically of the order $10^{-4}$ but is not limited to this where suitably designed material for the substrate could enhance this by several orders of magnitude. Using an asymmetric structure for the waveguide also increases the influence of $Q$. Here in these initial results the various parameters are selected for the simulations (with the appropriate values been given with individual simulations) in line with previous results obtain for temporal solitons.

In order to consider metamaterial effects on the NLSE breather type of input, the Crank-Nicolson method has been implemented in MATLAB to numerically solve the propagation equation. The developed code is capable of solving the extended NLSE as laid out in [120] which takes into account the appropriate effects of self-steepening and magnetooptic parameters. It requires an initial starting condition which then determines how this input condition will evolve as propagation occurs along $z$. As discussed in section 6.1 there is a very real choice of the kind of starting conditions that can be selected. Here in order to initiate a specific type of rogue wave condition we initially base our input on the solution to the standard NLSE determined by Peregrine which for the standard NLSE gives rise to a solitary pulse on an almost flat background. Following this we go on to look at a non-ideal starting condition which is discussed in more detail below.

The Peregrine solution can of course be used to describe the evolution of a single peak analytically, however by using a known solution as an input condition it allows the validity of the numerical solver to be checked for the case where there are no additional terms in the NLSE. Further, having done this, it then allows us see how the evolution of such a well known input is changed in the presence of metamaterials and magnetooptics by re-introducing the additional terms in the extended NLSE.

The exact Peregrine solution to the NLSE is $\psi=\left[1-4(1+2 \mathrm{i} z) /\left(1+4 t^{2}+4 z^{2}\right)\right] \mathrm{e}^{\mathrm{i} z}$ which has the characteristic that it peaks at $z=0$, both before and after this value the peak decays rapidly. If a specific value of $z$ is now selected, for example $z=-10$, it can then be used as a starting condition for the numerical solver which in the absence of additional terms in the NLSE should give a similar solution to the analytical approach.

Hence first we check the validity of our numerical solver for this new type of input and align it with previous work such as [96] by selecting the exact solution at $z=-10$ along the $z$-axis prior to the peak of the breather and allowing it to propagate along $z$. It can be clearly seen from figure 25 that 
(a)

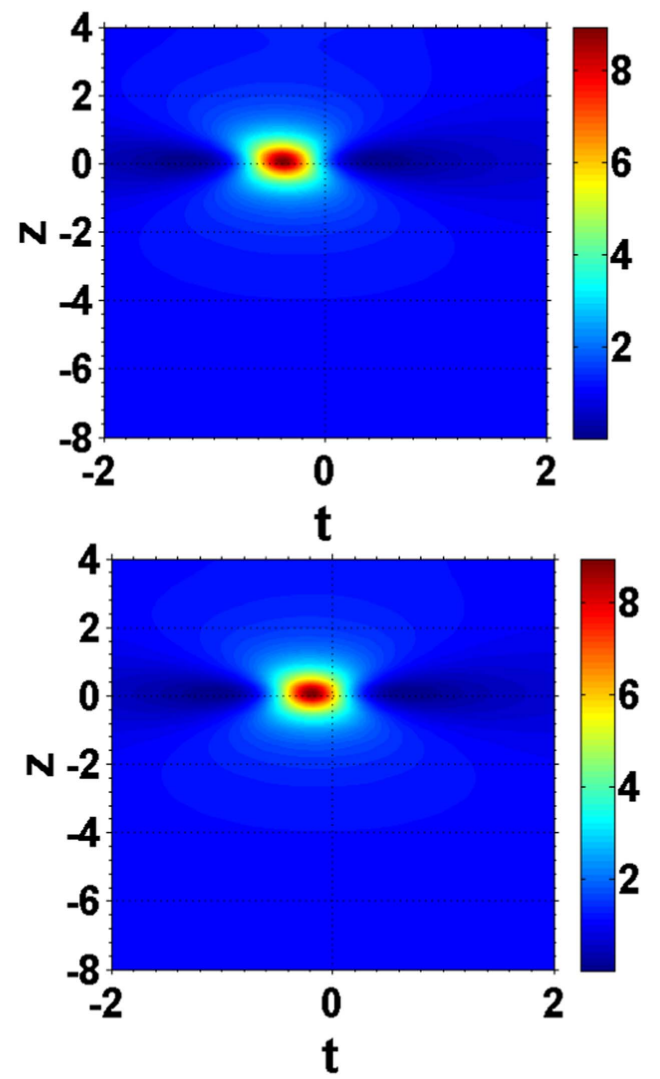

(c) (b)
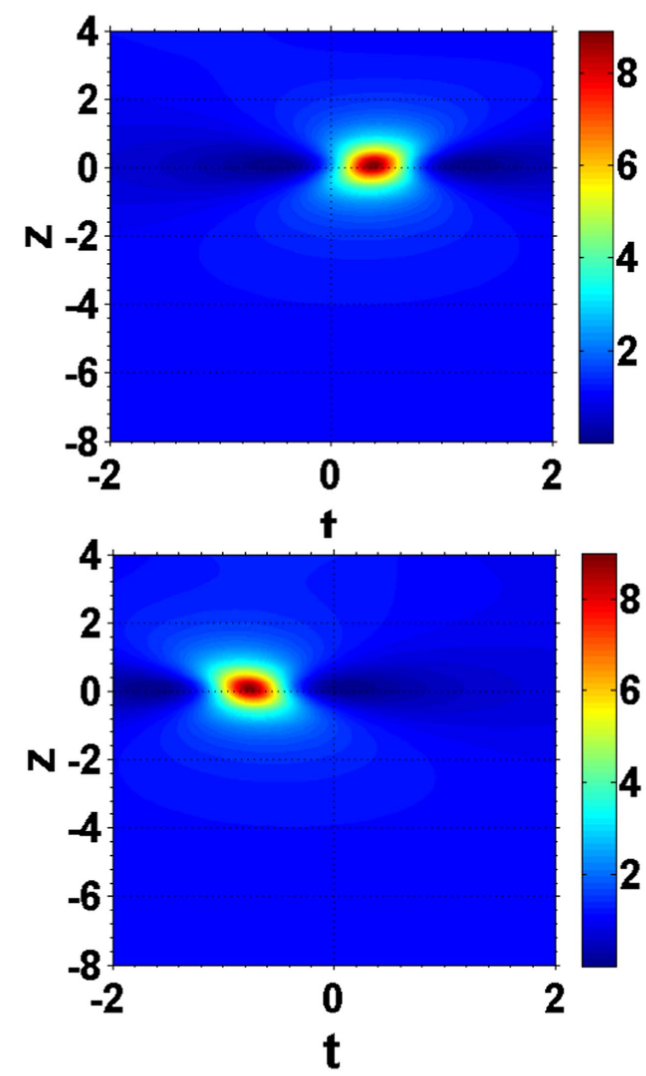

(d)

Figure 26. Effect of self-steepening on the excitation of the NLSE Peregrine soliton using a value of (a) $S=-0.02$, (b) $S=+0.02$, (c) $S=-0.01$, and (d) $S=-0.04$. Note the opposite time shifting of the intensity peak of near-Peregrine soliton for opposite values of $S$. As in figure 25 the darker areas to the side represent the points where the value of $|\psi|^{2}$ drops below 1 . The inital starting condition also gives a very small deviation from 1 at $z=-8$, which accounts for the almost flat appearance of the initial starting condition.

the result of this process is indeed a single growth/decay cycle centred at position $z=0$ in agreement with the analytical solution. Note that all figures shown below are pseudocolour plots showing the evolution of wave intensity $|\psi|^{2}$ as a function of normalised distance and time in order to highlight breather peak localisation. The variation of $|\psi|^{2}$ from a value of 1 is small at $z=-10$ and cannot easily be distinguished in the plots, however it is still present and significant as without it there would be no peak at $z=0$.

Subsequently in order to consider metamaterial effects the parameters that are adjusted here are as given above (see also [120]), which are: $S$ the self-steepening coefficient, $t_{\nu}$ the delay of the magnetisation after excitation by the electromagnetic input, $\Delta t_{\nu}$ the normalised time over which the magnetisation takes place, $v_{g}$ the group velocity and $\nu_{\max }$, the maximum value of magnetisation

Having established the Peregrine solution the next step is to introduce self-steepening. The self-steepening can be negative or positive, and it is shown that it is possible to arrange for the peak of the Peregrine to appear in different time slots depending on the values selected for the self-steepening coefficient.
As a starting point the values selected for the self-steepening coefficient $S$ are in line with those of [120]. As already stated above, a slight disturbance of the ideal NLSE propagation induces a deviation from the expected theoretical solution, but as shown in figure 26 most of features related to the Peregrine soliton remain clearly observable, i.e. the main features of a localised high amplitude event (i.e., rogue wave) still occur with similar peak power. It is also interesting to note that by using $S=-0.02$ in the simulation the time shift on the near-Peregrine soliton is of the same order of the effect on the standard soliton pulse, see figure 8(a) in [120]. Figure 26(a) illustrates this effect, the Peregrine soliton peak is shifted away from zero on the time axis by $\sim-0.45$ (for a distance $\Delta z=8$ ). Figure 26(b) shows the shift with $S=+0.02$ which is then shifted to the opposite side of zerotime line. Figure 26(c) shows a smaller shift from $S=-0.005$ and figure 26(d) has a larger shift resulting from a value for $S=-0.04$. This kind of tilted Peregrine soliton structure was also observed in the case of approximate polynomial rogue-wave solutions obtained beyond the integrable Sasa-Satsuma or Hirota equations [111]. This rogue wave structure makes an angle $\theta$ with the $z$-axis, which 


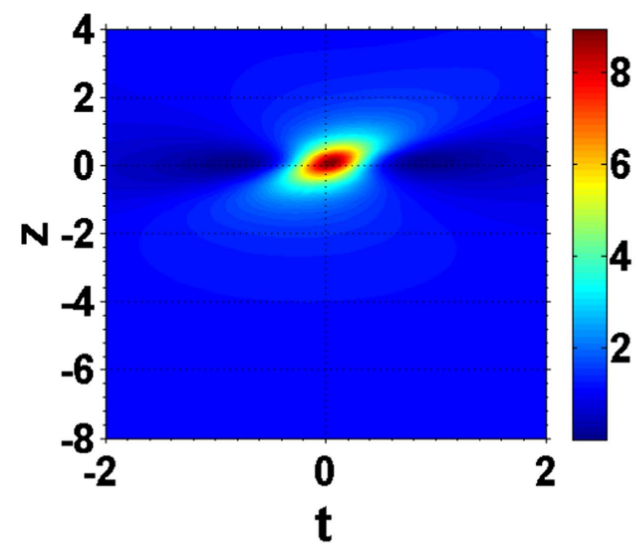

(a)

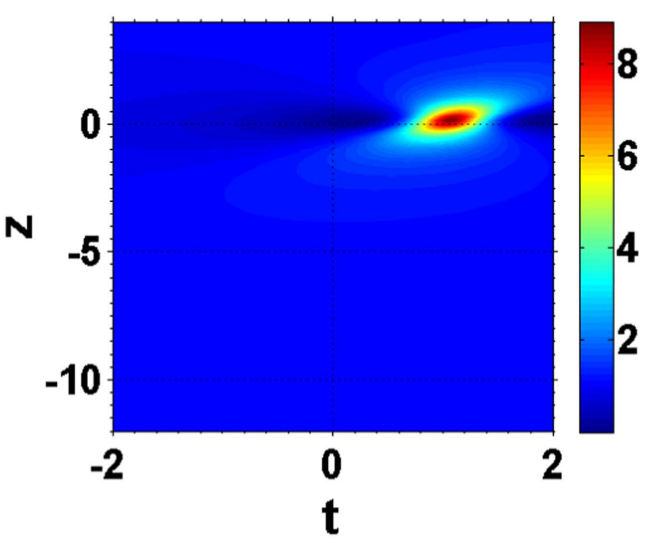

(b)

Figure 27. (a) Effect of magnetooptic parameters set as $\nu_{\max }=-10, t_{\nu}=200, \Delta t_{\nu}=10$ and $v_{g}=0.03$ on the self-steepening $S=-0.02$. The addition of the magnetooptics has moved the peak back towards the origin of the time axis (see figure 26(a)). (b) Same parameters but the difference lies in the distance propagated prior to the peak localisation. Here the starting point for the input is at -12 rather than -8 it can be seen that the magnetooptic effect now moves the peak to the positive part of the time axis. The small deviation of the starting of $|\psi|^{2}$ from 1 again gives the appearance of an almost flat initial condition. The dark areas either side of the main peak are again present where the value of $|\psi|^{2}$ drops below 1 .

corresponds to an effective velocity proportional to the selfsteepening coefficient $S$ [111].

At this point magnetooptic effects are introduced. This, as discussed previously is enabled through the values given for $t_{v}, \Delta t_{v}$ and $\nu_{\max }$. It is also noted that with the parameters set, for the magnetooptic effect to operate on the central peak of the Peregrine soliton, then there needs to be sufficient propagation distance prior to where the peak manifests itself. Figure 27(a) shows the effect of using a value of $S=-0.02$ with magnetooptic parameters set as $\nu_{\max }=-10, t_{\nu}=200$, $\Delta t_{\nu}=10$ and $v_{g}=0.03$. It can be seen that the addition of the magnetooptic parameters moves the peak back to the position $t=0$. It is noted that there is an overall clockwise rotation of the rogue wave structure. It is further noted that if a greater lead in distance is used then a lower value of $\nu_{\max }$ can be used to achieve a similar response. Alternatively a larger effect can be achieved with the same value of $\nu_{\max }$. Figure 27(b) shows the influence of the same magnetic parameters but with the propagation prior to the main peak being 12 dimensionless units along the $z$-axis rather than 8 . It is thus demonstrated that a significant controllable effect can be brought about on the near-Peregrine soliton through metamaterial effects, without cancelling the main features of the rogue wave solution. This of course is only an initial early stage result but the work is ongoing and it is intended to report further work in the near future.

As discussed above there are broader categories of solution to the NLSE that can be studied; for example the Akhmediev breather solution is one of these. This category of solution has, for example, been studied in [123] and gives multiple peaks in the time domain. However of particular interest here are non-exact types of breather solutions mentioned above, that to a large extent can reproduce the exact solution for the Akhmediev breathers but also tend to produce additional features.

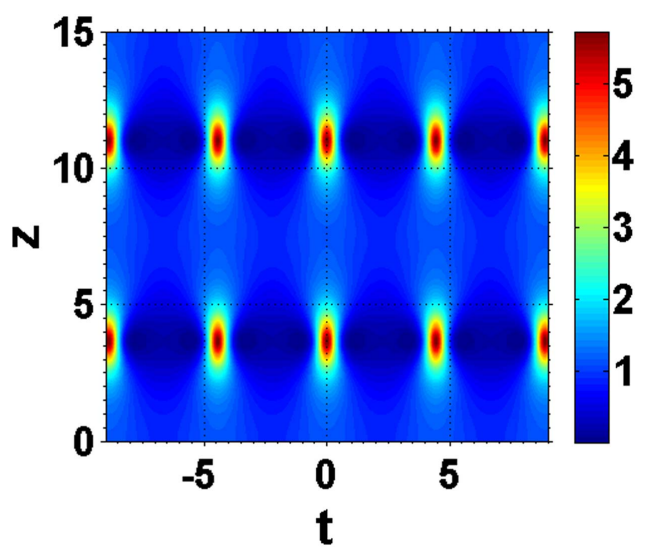

Figure 28. Simulation of the standard NLSE with the following input condition $\psi=\sqrt{1+0.145 \cos (\omega t)}$. The periodic emergence of near-Akhmediev breathers correlates well with figure 7(b) from [123]. Note the first row of peaks is what would be expected from an exact analytical solution. Here however, as in [123], the chosen nonexact input condition produces two rows of peaks. As with previous plots the darker areas to the sides of the peaks represent $|\psi|^{2}$ dropping below a value of 1 .

Hence we now investigate the excitation of NLSE breather solutions with non-ideal input conditions. Our objective is to assess the impact of negative phase nonlinear metamaterials in this area, in particular to extend the conclusions drawn for optical fibre platforms [96, 123, 124] in the presence of self-steepening and the addition of magnetooptic parameters. This is carried out in a similar manner to the process adopted for the exact solution simulations discussed above.

Here again the first step is to ensure correlation between the simulations previously carried out for the standard NLSE with non-ideal inputs [123] and the new simulator with metamaterial extensions. Figure 28 shows a test simulation carried out with the following input condition: $\psi=\sqrt{1+0.145 \cos (\omega t)}$ as 


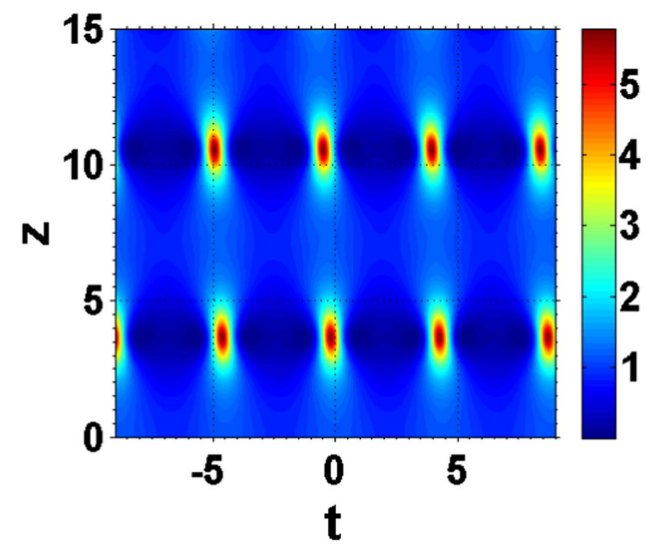

(a)

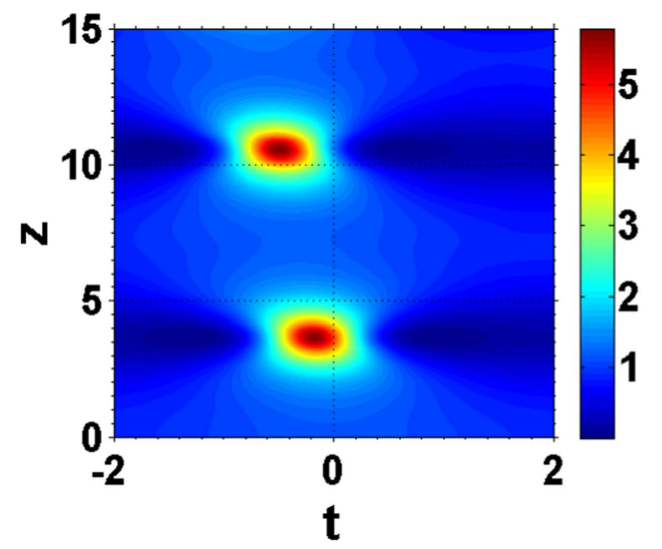

(b)

Figure 29. (a) Impact of self-steepening (with $S=-0.02$ ) on the non-ideal excitation of a near-Akhmediev breather $(a=0.25)$. The input condition is $\psi=\sqrt{1+0.145 \cos (\omega t)}$. It can be seen that both sets of peaks have moved along the time axis in a negative direction. (b) Expanded plot of the central portion of figure 29(a). On both plots the darker areas to the side of the main peaks show the value of $|\psi|^{2}$ droping below a value of 1 .

originally given in figure 7 from [123] where $\omega=2(1-2 a)^{1 / 2}$ and $a=0.25$. This input condition simply corresponds to an intensity-modulated continuous wave with angular frequency $\omega$ linked to the governing parameter $a$ (recall that $a$ determines the physical behaviour of the excited breather solution, the excited breather is part of the family of Akhmediev breathers). At this point the simulation does not contain any extensions to the NLSE. We observe that non-ideal initial conditions yield periodic evolution as a function of propagation in contrast to the exact Akhmediev-breather ( $\mathrm{AB}$ ) theory with a single growth/ decay cycle of the temporal periodic pattern. However, each growth-return cycle remains well-described by the analytic $\mathrm{AB}$ solution.

Having established a correlation between this and previous work, the self-steepening effect is now added to the simulation $(S=-0.02)$. Figure 29 shows such a scenario where it can be seen that both the first and second set of peaks have been rotated and moved in a negative direction with respect to the time axis. The second set have moved by approximately -0.6 on the normalised time axis for a propagation length $\Delta z=10.5$. This corresponds to a slightly longer propagation distance from the input than that in figure 26(a) for the exact solution where there is a slightly smaller shift. The first set of peaks shifts by approximately -0.2 or less, for a significantly shorter propagation distance from the input $(\Delta z=3.5)$. It can thus be seen that the effective velocity induced by self-steepening correlates well with different input conditions. It is noted that the shift leaves both sets of peaks aligned in the same orientation and rotated counter clockwise. Moreover the spatial localisation of the maximum intensity peak does not change significantly.

Figure 30 shows the effect of adding magnetooptic parameters with similar values to that used in figure 27 $\left(\nu_{\max }=-10, t_{\nu}=200, \Delta t_{\nu}=10\right.$ and $\left.v_{g}=0.03\right)$. It can be seen that the first set of peaks has not reached its original position prior to the addition of self-steepening and magnetooptic effects and still has counter clockwise rotation

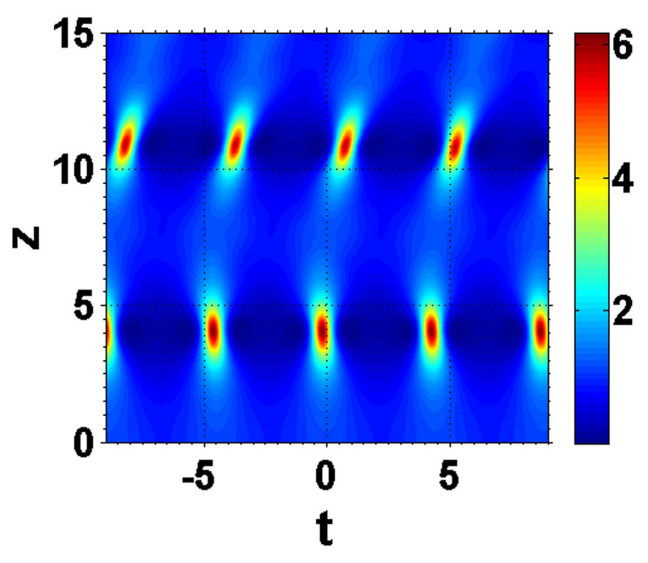

Figure 30. Impact of self-steepening and magnetooptic parameters on the non-ideal excitation of a near-Akhmediev breather $(a=0.25)$. The input condition is $\psi=\sqrt{1+0.145 \cos (\omega t)}$.

Similar values are used to those selected for figure $27\left(\nu_{\max }=-10\right.$, $t_{\nu}=200, \Delta t_{\nu}=10$ and $\left.v_{g}=0.03\right)$. There is a clear distinction between how the first and second sets of peaks respond due to the position along $z$. Alongside the peaks, as in previous plots there continues to be darker areas on the plot where $|\psi|^{2}<1$.

whereas the second set of peaks has moved well past the original position with a clockwise rotation. This again illustrates here that the magnitude of the response from the magnetooptics is position dependent.

Figure 31 has similar input parameters to figure 30 but importantly with $\nu_{\max }=-4$ rather than -10 . This has the effect of moving the central point of the second set of peaks back to the starting position prior to the addition of selfsteepening and magnetooptics. However as with previous simulations there is a small clockwise rotation as there is a greater effect of the magnetoptic addition the further along $z$. It also raises the possibility of having different magnetooptic parameters in different parts of the guide to allow for similar shifts on the time axis for different distances. Here we are able to almost cancel the effective velocity induced by self-steepening with an appropriate magnetooptic response. 


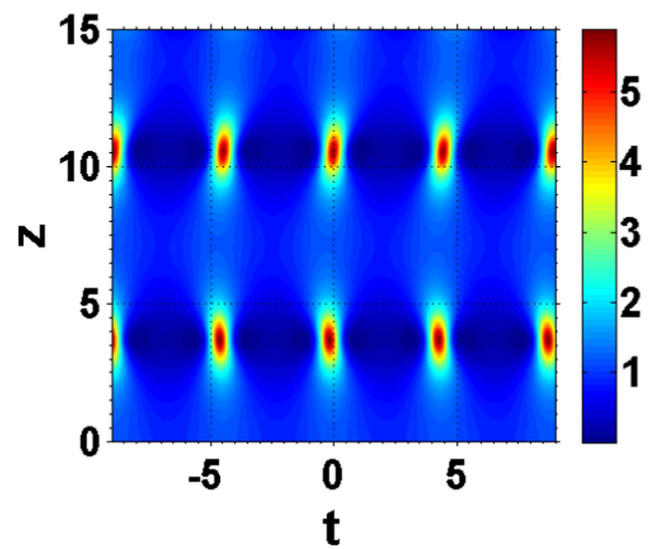

(a)

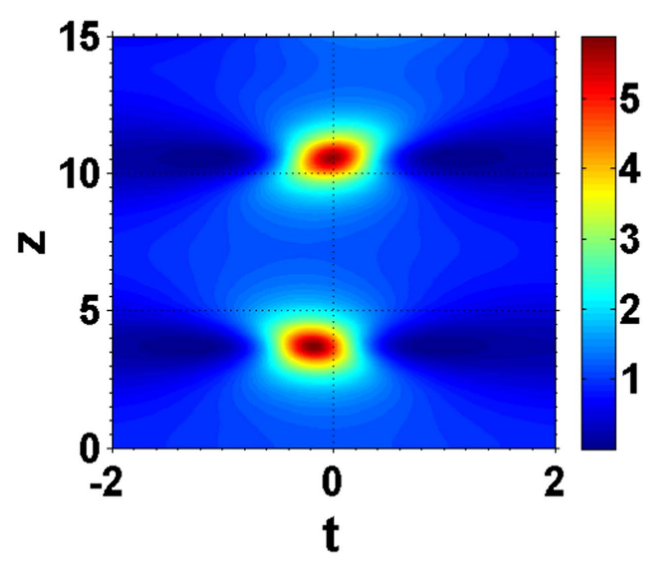

(b)

Figure 31. (a) Effect of magnetooptic parameters set as $\nu_{\max }=-4, t_{\nu}=200, \Delta t_{\nu}=10$ and $v_{g}=0.03$ on the self-steepening $S=-0.02$. The second set of pulses is moved back toward the original starting position by the addition of magnetoptic parameters and after being moved away by the effect of self-steepening. (b) Expanded plot of the central portion of figure 31(a). On both plots it can be observed alongside the peaks there continues to be darker areas that represent $|\psi|^{2}<1$.

These latter results with the use of non-exact input conditions thus show similar responses to the exact breather excitation. It is thus possible to see how a range of different input conditions could potentially be controlled. Although not yet fully optimised the parameters used here indicate that reasonably accurate control can be achieved that would allow useful systems to be developed. There are now a wide range of possibilities in terms of self-steepening, magnetooptic and other parameters that are now being researched for this purpose. The fact that breathers propagating with a certain angle to the line $t=0$ can also be induced by non-ideal input wave excitation, as well as propagation effects, is interesting to note [123]. The resulting inclined trajectory is usually associated with an asymmetric spectrum or/and frequency detuning, with a distinct mean group velocity of the breather under study. Note that rogue wave solutions analysed in the framework of the Sasa-Satsuma equation already revealed strong spectral asymmetries [125]. Again a detailed analysis of spectral evolution will be mandatory in future works, this information is important for a complete investigation of the rogue wave properties.

\subsection{Conclusions}

To conclude this section, we investigated the propagation behaviour of wave excitation corresponding to rogue NLSE breather solutions in transparent double-negative metamaterials, wherein higher-order dispersive or nonlinear effects are included. An appropriate extended NLSE was used which is not integrable. It takes into account typical higher order effects from metamaterials, such as the self-steepening and the magnetooptics parameters. We revealed the impact of the self-steepening on both Peregrine and Akhmediev breathers as the change of the mean group velocity of the evolving breather under study. Such time-shifting signatures can be also found in integrable systems such as the Sasa-Satsuma or the Hirota equations. With the application of magnetooptic influence/control over these phenomena, we demonstrated that the impact of self-steepening on breather waves can be cancelled or overcome, similarly to studies of the standard NLSE soliton. Most importantly, we confirmed that the extraterms studied here to the NLSE do not prevent the emergence of rogue wave structures almost identical to rogue NLSE breather solutions. Conversely, they offer a possible management of their unique pulsating dynamics and spatiotemporal localisation properties.

\section{Towards the future}

All of the investigations presented here offer some interesting perspectives on the interaction of electromagnetics and metamaterials. One broadly interesting example is illustrated in the final section, where it is demonstrated that promising responses can be elicited through the propagation of rogue waves in metamaterials under magnetooptic influence. It is important to emphasise, however, that there are many other possibilities to consider, as indeed this article shows. Hence, the whole material presented here provides starting points for some exciting new research areas and ways forward become apparent.

The discussions given here show that one of the great advantages that metamaterials offer is the potential to introduce novel and interesting properties into electromagnetic environments. These can be conveniently simulated by additions to the standard NLSE including, for example, quintic nonlinearity, the influence of Raman scattering, and the impact of magnetooptic effects. As shown earlier a strong application is the adaptation of rogue waves propagating in metamaterial environments. Several studies of suitable modifications to the NLSE $[117,120]$ for this type of propagation have emerged, already, for exploring aspects other than magnetooptic interaction with rogue waves, or near-rogue waves. For the latter, any modifications to the NLSE do need, however, to be considered in conjunction with various 
different classes of solutions particularly those with non-ideal starting conditions that were outlined above but also with other variants of non-ideal starting conditions that have yet to be explored. Future work also needs to include a detailed analysis of spectral evolution, this information is important for a complete investigation of the rogue wave properties under the various conditions discussed.

The list of possible future work does not stop there, because consideration needs to be given to other types of metamaterials. To date the concentration has been on double negative metamaterials, but the use of hyperbolic metamaterials is now a key ingredient in the way forward and a lot of the discussion in other sections covers this topic.

The advantages of hyperbolic media over double negative metamaterials includes the ease of construction at the nanoscale and their non-resonant nature, which is extremely important for broadband features. Beautiful materials like graphene can be used and the role of the position of the optical axis can be investigated thoroughly for future designs [50].

Hyperbolic metamaterial can be designed to operate over a broad range of frequency bands and open up the possibility of many other important features they can, for example, be shaped into very sub-wavelength cavities [126, 127]. However, in order to create designs for this class of metamaterials, simulations will require an even more general NLSE. In fact a range of different aspects of this work having been carried out in this area by, for example [50, 73]. The work presented here does not yet take us to the creative position we have for double negative media. For hyperbolic metamaterials to be considered, in the generation of rogue waves, for example, with magnetooptic control a lot of further work is needed and this is a major definition of the future of this area.

The literature shows that the global work to date has been with temporal solutions however there are spatial solutions that can also be studied along with all the possibilities outlined. In addition, as discussed, there are authors who have used transmission lines as metamaterials [118, 119] and it may be interesting to consider how this type of medium could be taken forward. There is thus an extensive list of options that can be studied in future programmes of work, giving rise to a very exciting way forward with the line of research discussed.

\section{Acknowledgments}

The investigations by $\mathrm{Y}$ Rapoport are based upon work supported by the project 16BF051-02 of the Taras Shevchenko National University of Kyiv. A Alberucci. and G Assanto relied upon support from the Academy of Finland through the Finland Distinguished Professor grant no. 282858. B Kibler had support from the French project PIA2/ ISITE-BFC. J McNiff received strong support from Original Perspectives Ltd. A D Boardman received strong IT support from the University of Salford. Vincent Uzomah, University of Salford, provided some printing technical assistance.

\section{References}

[1] Walser R M 2001 Electromagnetic metamaterials Proc. SPIE $44671-15$

[2] Veselago V G, Braginsky L, Shklover V and Hafner C 2006 Negative refractive index materials J. Comput. Theor. Nanosci. 3 189-218

[3] Smith D R, Pendry J B and Wiltshire M C K 2004 Metamaterials and negative refractive index Science 4 788-92

[4] Boardman A D, King N and Velasco L 2005 Negative refraction in perspective Electromagnetics 25 365-89

[5] Engheta N and Ziolkowski R W 2006 Metamaterials (Piscataway, NJ, New York: IEEE, Wiley)

[6] Ferrari L, Wu C, Lepage D, Zhang X and Liu Z 2015 Hyperbolic metamaterials and their applications Prog. Quantum Electron. 40 1-40

[7] Agrawal G P 2001 Nonlinear Fiber Optics 3rd edn (San Diego: Academic)

[8] Remoissenet M 1996 Waves Called Solitons (Berlin: Springer)

[9] Infeld E and Rowlands G 2000 Nonlinear waves, solitons and chaos (Cambridge: Cambridge University Press)

[10] Garrett C and Gemmrich J 2009 Rogue waves Phys. Today 6262

[11] Peregrine D H 1983 Water waves, nonlinear Schrödinger equation and their solutions J. Aust. Math. Soc. B 25 16-43

[12] Wen S C, Xiang Y J, Su W H, Hu Y H, Fu X Q and Fan D Y 2006 Role of the anomalous self-steepening effect in modulation instability in negative-index material $O p t$. Express 14 1568-75

[13] Wen S C, Wang Y W, Su W H, Xiang Y J, Fu X Q and Fan D Y 2006 Modulation instability in nonlinear negativeindex material Phys. Rev. E 73036617

[14] Wen S C, Xiang Y J, Dai X Y, Tang Z X, Su W H and Fan D Y 2007 Theoretical models for ultrashort electromagnetic pulse propagation in nonlinear metamaterials Phys. Rev. A 75033815

[15] Xiang Y J, Wen S C, Dai X Y, Tang Z X, Su W H and Fan D Y 2007 Modulation instability induced by nonlinear dispersion in nonlinear metamaterials J. Opt. Soc. Am. B 24 3058-63

[16] Scalora M et al 2005 Generalized nonlinear Schrödinger equation for dispersive susceptibility and permeability: application to negative index materials Phys. Rev. Lett. 95 013902

[17] Boardman A D and King N 2009 Magneto-optics and the Kerr effect with ferromagnetic materials Tutorials in Complex Photonic Media ed M A Noginov et al (Bellington, WA: SPIE Press)

[18] Boardman A D and Xie M 2003 Magnetooptics-a critical review Introduction to Complex Mediums for Optics and Electromagnetics ed W S Weiglhofer and A Lakhtakia (Bellington, WA: SPIE Press) pp 197-219

[19] Boardman A D and Xie M 2002 Vector spatial solitons in complex magneto-optic waveguides J. Opt. Soc. Am. B 19 563-73

[20] Boardman A D, Xie M and Xie K 2003 Surface magnetooptic solitons J. Phys. D: Appl. Phys. 36 2211-7

[21] McGahan W A et al 1991 Optical and magnetooptical characterization of tbfeco thin-films in trilayer structures J. Appl. Phys. 69 4568-70

[22] Caloz C and Itoh T 2006 Electromagnetic Metamaterials: Transmission Line Theory and Microwave Applications (New Jersey: Wiley-IEEE Press)

[23] Joannopoulos J D, Johnson S G, Winn J N and Meade R D 2008 Photonic Crystals: Molding the Flow of Light (Princeton, NJ: Princeton University Press) 
[24] Atwater H A and Polman A 2010 Plasmonics for improved photovoltaic devices Nat. Mater. 9 205-13

[25] Ilic O, Kaminer I, Lahini Y, Buljan H and Soljacic M 2016 Exploiting optical asymmetry for controlled guiding of particles with light ACS Photon. 3 197-202

[26] Shelby R A, Smith D R and Schultz S 2001 Experimental verification of a negative index of refraction Science 292 77-9

[27] Valagiannopoulos C A 2013 How nonreciprocal is an effective permittivity matrix? Microw. Opt. Technol. Lett. 56 2018-21

[28] Wang Z, Shi L, Liu Y, Xu X and Zhang X 2015 Optical nonreciprocity in asymmetric optomechanical couplers $S c i$ Rep. 58657

[29] Reiskarimian N and Krishnaswamy H 2016 Magnetic-free non-reciprocity based on staggered commutation Nat. Commun. 711217

[30] Fleury R, Sounas D L, Sieck C F, Haberman M R and Alu A 2014 Sound isolation and giant linear nonreciprocity in a compact acoustic circulator Science 343 516-9

[31] Poddubny A, Iorsh I, Belov P and Kivshar Y 2013 Hyperbolic metamaterials Nat. Photon. 7 958-67

[32] Drachev V P, Podolskiy V A and Kildishev A V 2013 Hyperbolic metamaterials: new physics behind a classical problem Opt. Express 21190862

[33] Noginov M A, Barnakov Y A, Zhu G, Tumkur T, Li H and Narimanov E E 2009 Bulk photonic metamaterial with hyperbolic dispersion Appl. Phys. Lett. 94151105

[34] Cortes C L, Newman W, Molesky S and Jacob Z 2012 Quantum nanophotonics using hyperbolic metamaterials J. Opt. 14063001

[35] Biehs S A, Tschikin M and Ben-Abdallah P 2012 Hyperbolic metamaterials as an analog of a blackbody in the near field Phys. Rev. Lett. 109104301

[36] Guo Y, Cortes C L, Molesky S and Jacob Z 2012 Broadband super-Planckian thermal emission from hyperbolic metamaterials Appl. Phys. Lett. 101131106

[37] Valagiannopoulos C A, Mirmoosa M S, Nefedov I S, Tretyakov S A and Simovski C R 2014 Hyperbolicmetamaterial antennas for broadband enhancement of dipole emission to free space J. Appl. Phys. 116163106

[38] Lu D, Kan J J, Fullerton E E and Liu Z 2014 Enhancing spontaneous emission rates of molecules using nanopatterned multilayer hyperbolic metamaterials $\mathrm{Nat}$. Nanotechnol. 9 48-53

[39] Caldwell J D et al 2014 Sub-diffractional volume-confined polaritons in the natural hyperbolic material hexagonal boron nitride Nat. Commun. 55221

[40] Valagiannopoulos C A and Simovski C R 2011 Conversion of evanescent waves Into propagating modes by passing through a metamaterial prism: an iterative approximation method Proc. 5th European Conf. on Antennas and Propagation (EUCAP) (Rome, Italy)

[41] Valagiannopoulos C A and Tretyakov S A 2014 Emulating hyperbolic-media properties with conventional structures New J. Phys. 16063004

[42] Kidwai O, Zhukovsky S V and Sipe J E 2011 Dipole radiation near hyperbolic metamaterials: applicability of effectivemedium approximation Opt. Lett. 36 2530-2

[43] Kidwai O, Zhukovsky S V and Sipe J E 2012 Effective-medium approach to planar multilayer hyperbolic metamaterials: strengths and limitations Phys. Rev. A 85053842

[44] Geim A K and Novoselov K S 2007 The rise of graphene Nat. Mater. 6 183-91

[45] Novoselov K S et al 2007 Electronic properties of graphene Phys. Status Solidi b 244 4106-11

[46] Hanson G W 2008 Dyadic Green's functions and guided surface waves for a surface conductivity model of graphene J. Appl. Phys. 103064302
[47] Vakil A and Engheta N 2011 Transformation optics using graphene Science 332 1291-4

[48] Othman M A K, Guclu C and Capolino F 2013 Graphenedielectric composite metamaterials: evolution from elliptic to hyperbolic wavevector dispersion and the transverse epsilonnear-zero condition J. Nanophoton. 7073089

[49] Iorsh I V, Mukhin I S, Shadrivov I V, Belov P A and Kivshar Y S 2013 Hyperbolic metamaterials based on multilayer graphene structures Phys. Rev. B 87075416

[50] Boardman A D, Egan P and McCall M 2015 Optic axisdriven new horizons for hyperbolic metamaterials EPJ Appl. Metamater. 211

[51] Hashemi S M, Nefedov I S and Soleimani M 2013 Waves in asymmetric hyperbolic media Photon. Lett. Poland 5 72-4

[52] Nefedov I S and Melnikov L A 2014 Super-Planckian farzone thermal emission from asymmetric hyperbolic metamaterials Appl. Phys. Lett. 105161902

[53] Popov A K, Shalaev M I, Myslivets S A, Slabko V V and Nefedov I 2012 Enhancing coherent nonlinear-optical processes in nonmagnetic backward-wave materials Appl. Phys. A 109 835-40

[54] Popov A K, Shalaev M I, Slabko V V, Myslivets S A and Nefedov I 2012 Nonlinear backward-wave photonic metamaterials Adv. Sci. Technol. 77 246-52

[55] Popov A K, Slabko V V, Shalaev M I, Nefedov I and Myslivets S A 2014 Nonlinear optics with backward waves: extraordinary features, materials and applications Solid State Phenom. 213 222-5

[56] Valagiannopoulos C A and Nefedov I S 2013 Increasing the electromagnetic attenuation below a quasi-matched surface with use of passive hyperbolic metamaterials Photon. Nanostruct.: Fundam. Appl. 11 182-90

[57] Hashemi S M and Nefedov I S 2012 Wideband perfect absorption in arrays of tilted carbon nanotubes Phys. Rev. B 86195411

[58] Nefedov I S, Valagiannopoulos C A, Hashemi S M and Nefedov E I 2013 Total absorption in asymmetric hyperbolic media Sci. Rep. 32662

[59] Nefedov I S, Valagiannopoulos C A and Melnikov L A 2013 Perfect absorption in graphene multilayers J. Opt. 15114003

[60] Mattheakis M, Valagiannopoulos C A and Kaxiras E 2016 Epsilon-near-zero behavior from plasmonic Dirac point: theory and realization using two-dimensional materials Phys. Rev. B 94201404

[61] Smolyaninov S and Yu-Ju H 2011 Modeling of time with metamaterials J. Opt. Soc. Am. B 28 1591-5

[62] Shekhar P, Jonathon A and Jacob Z 2014 Hyperbolic metamaterials: fundamentals and applications Nano Convergence 414

[63] Boardman A D, Egan P and McCall M 2015 Optic axisdriven new horizons for hyperbolic metamaterials EPJ Appl. Metamater. 2 1-7

[64] Alberucci A and Assanto G 2011 Nonparaxial $(1+1) D$ spatial solitons in uniaxial media Opt. Lett. 36 193-5

[65] Rapoport Y G, Grimalsky V V, Koshevaya S V. Boardman A D and Malnev V N 2014 New method for modeling nonlinear hyperbolic concentrators IEEE 34th Int. Scientific Conf. on Electronics and Nanotechnology, ELNANO 2014-Conf. Proc. article number 6873975, pp 35-8

[66] Rapoport Y, Boardman A, Grimalsky V, Selivanov Y and Kalinich N 2012 Metamaterials for space physics and the new method for modeling isotropic and hyperbolic nonlinear concentrators 14th Int. Conf. on Mathematical Methods in Electromagnetic Theory, MMET 2012 (Kharkiv, Ukraine, 28-30 August 2012) category number CFP12761-CDR, 2012IEEE; art. no 6331154, pp 76-9

[67] Marchuk G I 1990 Splitting and alternating direction methods Handbook of Numerical Analysis ed P G Ciarlet and J L Lions vol 1 (Oxford: Elsevier) pp 197-462 
[68] Kivshar Y S and Agrawal G P 2003 Optical Solitons (New York: Academic) p 539

[69] Felsen L F and Marcivitz M 1973 Radiation and Scattering of Waves (Englewood Cliffs, NJ: Prentice-Hall) vol 2, p 551

[70] Samarskii A A 2001 The Theory of Difference Schemes (New York: Dekker) pp 543-642

[71] Rytov S M 1956 Electromagnetic properties of a finely stratified medium Zh. Eksp. Teor. Fiz. 29605

[72] Smith D R and Schurig D 2003 Electromagnetic wave propagation in media with indefinite permittivity and permeability tensors Phys. Rev. Lett. 90077405

[73] Alberucci A, Jisha C P, Boardman A D and Assanto G 2016 Anomalous diffraction in hyperbolic materials Phys. Rev. A 94033830

[74] Born M and Wolf E 1970 Principles of Optics (Oxford: Pergamon)

[75] Alberucci A and Assanto G 2011 On beam propagation in anisotropic media: one-dimensional analysis Opt. Lett. 36 334-6

[76] Piccardi A, Trotta M, Kwasny M, Alberucci A, Asquini R, Karpierz M, D'Alessandro A and Assanto G 2011 Trends and trade-offs in nematicon propagation Appl. Phys. B 104 805-11

[77] Ishii S, Kildishev A V, Narimanov E, Shalaev V M and Drachev V P 2013 Sub-wavelength interference pattern from volume plasmon polaritions in a hyperbolic medium Laser Photon. Rev. 7265

[78] Paul T, Rockstuhl C, Menzel C and Lederer F 2009 Anomalous refraction, diffraction, and imaging in metamaterials Phys. Rev. B 79115430

[79] Kou Y, Ye F and Chen X 2011 Multipole plasmonic lattice solitons Phys. Rev. A 84033855

[80] Smolyaninov I I 2013 Analog of gravitational force in hyperbolic metamaterials Phys. Rev. A 88033843

[81] Hasegawa A and Tappert F 1973 Transmission of stationary nonlinear optical pulses in dispersive dielectric fibers: I. Anomalous dispersion Appl. Phys. Lett. 23 142-4

[82] Hasegawa A and Tappert F 1973 Transmission of stationary nonlinear optical pulses in dispersive dielectric fibers: II. Normal dispersion Appl. Phys. Lett. 23 171-2

[83] Snyder A W, Mitchell D J, Poladian L and Ladouceur F 1991 Self-induced optical fibers: spatial solitary waves Opt. Lett. 16 21-3

[84] Peccianti M, Conti C, Assanto G, De Luca A and Umeton C 2004 Routing of anisotropic spatial solitons and modulational instability in liquid crystals Nature 432 733-7

[85] Lax M, Louisell W H and McKnight W B 1975 From Maxwell to paraxial wave optics Phys. Rev. A 11 1365-70

[86] Silveirinha M G 2013 Theory of spatial optical solitons in metallic nanowire materials Phys. Rev. B 87235115

[87] Li T and Khurgin J B 2016 Hyperbolic metamaterials: beyond the effective medium theory Optica 3 1388-96

[88] Argyropoulos C, Estakhri N M, Monticone F and Alú A 2013 Negative refraction, gain and nonlinear effects in hyperbolic metamaterials Opt. Express 2115037

[89] Yu K, Guo Z, Jiang H and Chen H 2016 Loss-induced topological transition of dispersion in metamaterials J. Appl. Phys. 119203102

[90] Orlov A A, Voroshilov P M, Belov P A and Kivshar Y S 2011 Engineered optical nonlocality in nanostructured metamaterials Phys. Rev. B 84045242

[91] Yan W, Wubs M and Mortensen N A 2012 Hyperbolic metamaterials: nonlocal response regularizes broadband supersingularity Phys. Rev. B 86205429

[92] Solli D R, Ropers C, Koonath P and Jalali B 2007 Optical rogue waves Nature 450 1054-7

[93] Akhmediev N and Pelinovsky E 2010 Discussion \& debate: rogue waves-towards a unifying concept? Eur. Phys. J. Spec. Top. $1851-258$
[94] Akhmediev N, Dudley J M, Solli D R and Turitsyn S K 2013 Recent progress in investigating optical rogue waves J. Opt. 15060201

[95] Onorato M, Residori S, Bortolozzo U, Montina A and Arecchi F T 2013 Rogue waves and their generating mechanisms in different physical contexts Phys. Rep. 528 47-89

[96] Kibler B, Fatome J, Finot C, Millot G, Dias F, Genty G, Akhmediev N and Dudley J M 2010 The Peregrine soliton in nonlinear fibre optics Nat. Phys. 6 790-5

[97] Chabchoub A, Hoffmann N P and Akhmediev N 2011 Rogue wave observation in a water wave tank Phys. Rev. Lett. 106 204502

[98] Zakharov V E and Shabat A B 1971 Exact theory of twodimensional self-focusing and one-dimensional selfmodulation of waves in nonlinear media Zh. Eksp. Teor. Fiz. 61118

Zakharov V E and Shabat A B 1972 Sov. Phys._JETP 3462

[99] Lighthill M J 1965 Contribution to the theory of waves in nonlinear dispersive systems J. Inst. Math. Appl. 1 269-306

[100] Whitham G B 1965 A general approach to linear and nonlinear dispersive waves using a Lagrangian J. Fluid Mech. 22 273-83

[101] Bespalov V I and Talanov V J 1966 Filamentary structure of light beams in nonlinear liquids JETP Lett. 3 307-10

[102] Benjamin T B and Feir J E 1967 The disintegration of wave trains on deep water: I. Theory J. Fluid Mech. 27 417-30

[103] Benjamin T B 1967 Instability of periodic wavetrains in nonlinear dispersive systems Proc. R. Soc. A 299 59-75

[104] Zakharov V E 1968 Stability of periodic waves of finite amplitude on a surface of deep fluid J. Appl. Mech. Tech. Phys. 9 190-4

Zakharov V E and Ostrovsky L A 2009 Modulation instability: the beginning Physica D 238 540-8

[105] Akhmediev N and Ankiewicz A 1997 Solitons, Nonlinear Pulses and Beams (London: Chapman and Hall)

[106] Osborne A R 2010 Nonlinear OceanWaves and the Inverse Scattering Transform (San Diego: Academic)

[107] Peregrine D H 1983 Water waves, nonlinear Schrödinger equations and their solutions J. Aust. Math. Soc. B 25 16-43

[108] Akhmediev N and Korneev V I 1986 Modulation instability and periodic solutions of the nonlinear Schrödinger equation Theor. Math. Phys. 69 1089-93

[109] Ankiewicz A, Devine N and Akhmediev N 2009 Are rogue waves robust against perturbations? Phys. Lett. A 373 3997-4000

[110] Agrawal G P 2013 Nonlinear Fiber Optics 5th edn (Oxford: Academic)

[111] Ankiewicz A, Soto-Crespo J M, Chowdhury M A and Akhmediev N 2013 Rogue waves in optical fibers in presence of third-order dispersion, self-steepening, and selffrequency shift J. Opt. Soc. Am. B 30 87-94

[112] Ankiewicz A, Soto-Crespo J M and Akhmediev N 2010 Rogue waves and rational solutions of the Hirota equation Phys. Rev. E 81046602

[113] Bandelow U and Akhmediev N 2012 Persistence of rogue waves in extended nonlinear Schrödinger equations: integrable Sasa-Satsuma case Phys. Lett. A 376 1558-61

[114] Chen S 2013 Twisted rogue-wave pairs in the Sasa-Satsuma equation Phys. Rev. E 88023202

[115] Veselago V G 1967 The electrodynamics and substances with simultaneously negative values of $\varepsilon$ and $\mu$ Sov. Phys.-Usp. 10 509-14

[116] Pendry J B 2000 Negative refraction makes a perfect lens Phys. Rev. Lett. 85 3966-9

[117] Essama B G O, Atangana J, Biya-Motto F, Mokhtari B, Eddeqaqi N C and Kofane T C 2014 Optical rogue waves generation in a nonlinear metamaterial Opt. Commun. 331 334-47 
[118] Dontsop P Y G, Essama B G O, Dongo J M, Dedzo M M, Atangana J, Yemele D and Kofane T C 2016 AkhmedievPeregrine rogue waves generation in a composite right/lefthanded transmission line Opt. Quantum. Electron. 4859

[119] Shen Y, Kevrekidis P G, Veldes G P, Frantzeskakis D J, DiMarzio D, Lan X and Radisic V 2017 From solitons to rogue waves in nonlinear left-handed metamaterials Phys. Rev. E 95032223

[120] Boardman A D, Hess O, Mitchell-Thomas R C, Rapoport Y G and Velasco L 2010 Temporal solitons in magnetooptic and metamaterials waveguides Photon. Nanostruct.-Fundam. Appl. 8 228-43

[121] Boardman A D, Mitchell-Thomas R C, King N J and Rapoport Y G 2010 Bright spatial solitons in controlled negative phase metamaterials Opt. Commun. 283 1585-97

[122] Scalora M et al 2005 Generalized nonlinear Schrödinger equation for dispersive susceptibility and permeability: application to negative index materials Phys. Rev. Lett. 95 013902

[123] Kibler B, Fatome J, Finot C and Millot G 2016 Rogue and Shock Waves (Lectures Notes in Physics vol 926) ed M Onorato (Berlin: Springer) p 89

[124] Akhmediev N et al 2016 Roadmap on optical rogue waves and extreme events J. Opt. 18063001

[125] Akhmediev N, Soto-Crespo J M, Devine N and Hoffmann N P 2015 Rogue wave spectra of the SasaSatsuma equation Physica D 294 37-42

[126] Yang X, Yao J, Rho J, Yin X and Zhang X 2012 Experimental realization of three-dimensional indefinite cavities at the nanoscale with anomalous scaling laws Nat Photon. 6 450-4

[127] Yao J, Yang X, Yin X, Bartal G and Zhang X 2011 Threedimensional nanometer-scale optical cavities of indefinite medium Proc. Natl Acad. Sci. 108 11327-31 